\title{
Transient and localized processes in the magnetotail: a review
}

\author{
A. S. Sharma ${ }^{1}$, R. Nakamura ${ }^{2}$, A. Runov ${ }^{2, *}$, E. E. Grigorenko ${ }^{3}$, H. Hasegawa ${ }^{4}$, M. Hoshino ${ }^{5}$, P. Louarn ${ }^{6}$, C. J. Owen ${ }^{7}$, \\ A. Petrukovich $^{3}$, J.-A. Sauvaud ${ }^{6}$, V. S. Semenov ${ }^{8}$, V. A. Sergeev ${ }^{8}$, J. A. Slavin ${ }^{9}$, B. U. Ö. Sonnerup ${ }^{10}$, L. M. Zelenyi ${ }^{3}$, \\ G. Fruit $^{6}$, S. Haaland ${ }^{11}$, H. Malova ${ }^{3}$, and K. Snekvik ${ }^{11}$ \\ ${ }^{1}$ Department of Astronomy, University of Maryland, College Park, USA \\ ${ }^{2}$ Space Research Institute, Austrian Academy of Sciences, Graz, Austria \\ ${ }^{3}$ Space Research Institute, Russian Academy of Sciences, Moscow, Russia \\ ${ }^{4}$ Institute of Space and Astronautical Science, Kanagawa, Japan \\ ${ }^{5}$ University of Tokyo, Tokyo, Japan \\ ${ }^{6} \mathrm{CESR} / \mathrm{CNRS}$, Toulouse, France \\ ${ }^{7}$ Mullard Space Science Laboratory, University College, London, UK \\ ${ }^{8}$ Institute of Physics, St. Petersburg State University, St. Petersburg, Russia \\ ${ }^{9}$ Laboratory for Extraterrestrial Physics, NASA GSFC, Greenbelt, USA \\ ${ }^{10}$ Thayer School of Engineering, Dartmouth College, Hanover, USA \\ ${ }^{11}$ University of Bergen, Bergen, Norway \\ * currently at: Institute of Geophysics and Planetary Physics, UCLA, Los Angeles, CA, USA
}

Received: 21 June 2007 - Revised: 29 January 2008 - Accepted: 3 March 2008 - Published: 13 May 2008

\begin{abstract}
Many phenomena in the Earth's magnetotail have characteristic temporal scales of several minutes and spatial scales of a few Earth radii $\left(R_{E}\right)$. Examples of such transient and localized mesoscale phenomena are bursty bulk flows, beamlets, energy dispersed ion beams, flux ropes, traveling compression regions, night-side flux transfer events, and rapid flappings of the current sheet. Although most of these observations are linked to specific interpretations or theoretical models they are inter-related and can be the different aspects of a physical process or origin. Recognizing the inter-connected nature of the different transient and localized phenomena in the magnetotail, this paper reviews their observations by highlighting their important characteristics, with emphasis on the new results from Cluster multipoint observations. The multi-point Cluster measurements have provided, for the first time, the ability to distinguish between temporal and spatial variations, and to resolve spatial structures. Some examples of the new results are: flux ropes with widths of $0.3 R_{E}$, transient field aligned currents associated with bursty bulk flows and connected to the Hall current at the magnetic reconnection, flappings of the magnetotail current sheet with time scales of $100 \mathrm{~s}-10 \mathrm{~min}$ and thickness of few thousand $\mathrm{km}$, and particle energization including velocity and time dispersed ion structures with the
\end{abstract}

Correspondence to: A. S. Sharma

(ssh@astro.umd.edu) latter having durations of 1-3 min. The current theories of these transient and localized processes are based largely on magnetic reconnection, although the important role of the interchange and other plasma modes are now well recognized. On the kinetic scale, the energization of particles takes place near the magnetic $\mathrm{X}$-point by non-adiabatic processes and wave-particle interactions. The theory, modeling and simulations of the plasma and field signatures are reviewed and the links among the different observational concepts and the theoretical frameworks are discussed. The mesoscale processes in the magnetotail and the strong coupling among them are crucial in developing a comprehensive understanding of the multiscale phenomena of the magnetosphere.

Keywords. Magnetospheric physics (Magnetotail; Plasma sheet; Plasma waves and instabilities)

\section{Introduction}

The Earth's magnetosphere is formed by the interaction of the solar wind with its dipole magnetic field. Its features, such as shape and size, depend on the solar wind plasma and magnetic field, and vary significantly. In particular, the stress applied by the solar wind on the Earth's magnetic field can lead to magnetic reconnection, which is the main coupling mechanism between the solar wind and the magnetosphere.

Published by Copernicus Publications on behalf of the European Geosciences Union. 


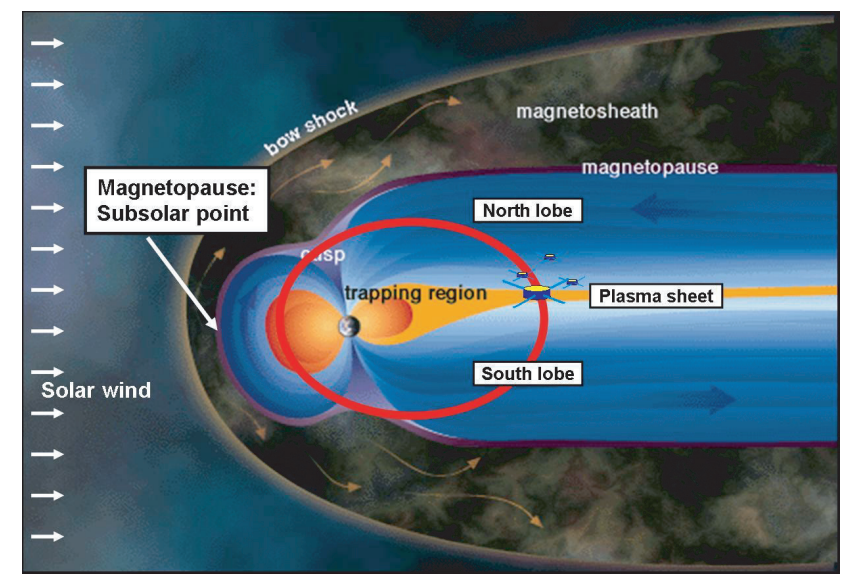

Fig. 1. A schematic view of the Cluster orbit when the apogee is in the magnetotail. Such orbits have occur during the summer season (Copyright:ESA).

When the interplanetary magnetic field (IMF) is southward and anti-parallel to the Earth's magnetic field, the two magnetic fields may reconnect at the dayside magnetopause and the consequent transport of magnetic flux to the tail lobes causes increased magnetic stress in that region. This build up of magnetic flux leads to reconnection in the magnetotail. The stored magnetic field energy in the magnetotail is released in an explosive manner, accompanied by a variety of signatures, including enhanced field-aligned currents, particle acceleration, and fast plasma flows. These processes involve many different spatial and temporal scales, and the broader origin of this multi-scale phenomena is the stronglycoupled nature of different processes in the different regions of the magnetosphere. Thin current sheets in the magnetotail are key boundary regions where the processes responsible for the onset of explosive release of energy take place, e.g., during substorms. Although the plasma processes in these current sheets are kinetic in nature, with scale sizes as short as the electron gyro radius, the strong cross-scale coupling drives more global magnetospheric features, such as plasmoid formation and release.

In the magnetotail, microscale processes, occuring on the electron or ion gyroradius scales, have corresponding time scales of less than a few seconds. On the other hand, largescale processes, which can be described typically by global MHD models, have time scales longer than tens of minutes. Between these two scales there is a number of processes whose spatial scales, at a few Earth radii $\left(R_{E}\right)$, are shorter than that of global processes and hence may be referred to as localized. These processes are essentially transient, with typical time scales of several minutes, compared to those of quasi-steady large-scale processes. Examples of these phenomena are bursty bulk flows (BBF) in the plasma sheet, flux ropes and traveling compression regions (TCR), nightside flux transfer events (NFTE), field-aligned current (FAC) filaments, velocity dispersed ion structures (VDIS), time dispersed ion structures (TDIS), beamlets at the lobe or plasma sheet boundary, and current sheet flapping and oscillations. These phenomena play key roles in magnetotail dynamics.

The Cluster mission, launched in summer 2000, consists of four identically instrumented satellites, and has provided an excellent opportunity for detailed investigation of these transient processes. The concept of the Cluster mission is described in Escoubet et al. (1997, 2001). The spacecraft separation, orbit, and instruments were selected to facilitate studies of plasma processes in key geospace regions, such as the bow shock, magnetopause, magnetotail, and auroral zone. The separations range from several hundred $\mathrm{km}$ up to several Earth radii. The orbit is polar, with $4 R_{E}$ perigee and $19.6 R_{E}$ apogee, both roughly in the magnetospheric equator. The orbital plane rotates in local time over one year, so that six months after apogee is on the day side, it is on the nightside. A typical Cluster orbit with apogee in the magnetotail is shown in Fig. 1. Most of the Cluster observations discussed in this study took place during such orbital conditions. Each Cluster satellite carries the same set of eleven instruments that allow for the detection of electron and ion distribution functions at spin $(4 \mathrm{~s})$ resolution, and the measurement of electric and magnetic fields at higher time resolution, from DC to high frequencies.

This paper is a review of the current understanding of magnetotail transient processes and localized structures, i.e., those which are mesoscale in both in space and time, with emphasis on the results from Cluster observations, and the new analysis techniques, models and underlying theories. In the next two sections we describe first the observational features followed by the theory, modeling and simulations of these phenomena. The review of the theory and modeling in Sect. 3 is motivated by the observations reviewed in Sect. 2, and is not exhaustive. In Sect. 4 we discuss the unsolved complexities and problems in studying these phenomena. The paper concludes with a summary of the current status and outlook in the understanding of these mesoscale and related phenomena.

\section{Observations}

This section reviews the current understanding of transient and localized processes in the magnetotail from observations. A few words of caution are however appropriate at the outset. First, either a transient process, or a passage of a localized structure with the required scale, may typically generate similar variations, whose origin, temporal or spatial, is difficult to distinguish using observations made with a single spacecraft. Recent multi-spacecraft observations provided by Cluster contribute significantly to resolving this issue. However much work has still to be done. Second, most material in this section concerns the mid-tail regime 
(well-developed current sheet, small $B_{z}$ ), rather than covering distant tail or the inner dipole-like regions.

The different types of mesoscale transients which have appeared in the literature are presented in the following subsections. In each case we present a typical example, discuss characteristic parameter values and behavior, highlight relevant Cluster results, and give brief references to possible interpretations described in more details in Sect. 3. It should be noted that seemingly similar variations of physical parameters can be interpreted in a number of different ways, and thus appear under different names in different subsections. For example, the perturbations related to traveling compression regions (TCR), nightside flux transfer events (NFTE) and "current disruption" (CD) in Sects. 2.3, 2.4 and 2.6, respectively, share similar characteristics. Another problem is that a specific name has been closely linked with one specific interpretation, which may not be unique in reality. For example, the "flux rope signatures" may be formed by localized transient reconnection (Sect. 3.2), by multiple reconnection (Sect. 3.3) or by the field-aligned current filaments (Sect. 2.5), in addition to the classical flux rope interpretation (Sect. 2.2).

\subsection{Bursty Bulk Flows}

Bursty Bulk Flows (BBF) are strong, transient and localized increases of plasma transport observed in the plasma sheet. They are a basic element of magnetotail dynamics and constitute an important cornerstone in the understanding of transient localized structures. Their formal definition includes transport rate increases by an order of magnitude above the average value (using, e.g., either bulk flow $V>400 \mathrm{~km} / \mathrm{s}$ (Angelopoulos et al., 1992), or flux transport along the tail $\sim V_{x} B_{z}>2 \mathrm{mV} / \mathrm{m}$ in the case of Rapid Flux Transfer (RFT) events introduced by Schödel et al., 2001). Hereafter we use the name BBF for such events, irrespective of their details. In the probability distribution of plasma flows they appear as the high-speed non-Gaussian tail in the distribution, and although they are only $\sim 5 \%$ of all samples they provide a dominant (70-80\%) contribution to the total plasma sheet transport. The predominant component of BBF velocity is $V_{x}$ (Baumjohann, 1993), which is along the tail axis and is consistent with the average $\mathbf{j} \times \mathbf{B}$ force direction in the tail plasma sheet. They are convective flows in the central plasma sheet (CPS), the high beta region of the plasma sheet, and distinct from the flows in the plasma sheet boundary layer (PSBL), where the ion beams are frequently observed and bulk flows are often predominantly field-aligned (e.g. Raj et al., 2002; Petrukovich et al., 2001). In the following, the BBF properties in the central plasma sheet region are reviewed.

The relationship of BBFs to the plasma sheet transients are characterized by three features: temporal scale, spatial localization scales, and plasma/structural properties. Before discussing these in detail, we note the close association of BBFs with the optical auroras, which was confirmed in many studies of isolated BBFs or isolated breakups (Ieda et al., 1998; Zesta et al., 2000; Sergeev et al., 2000a; Nakamura et al., 2001a; Sergeev, 2004) and provides the only source of information about the global aspects of BBFs (e.g., configuration, global development, lifetime, etc.). Among these, the auroral streamers, which are optical structures developing from poleward oval to equatorward oval, often aligned along the north-south direction, are the clearest and most identifiable BBF-related auroral form.

BBFs are highly fluctuating, and both the spatial effects (transport of plasma sheet structures with the fast flow) and the true transient effects (temporal variations of the source, together with temporal evolution of transported structures) contribute to this, making separation of their true sources difficult. Angelopoulos et al. (1992, 1994) (also Schödel et al., 2001) noted that the entire BBF has a typical duration of $\sim 10 \mathrm{~min}$, but is composed of superimposed $\sim 1$ min long bursts. (The average duration can double if the observations at 3 Cluster spacecraft separated by less than $2000 \mathrm{~km}$ (e.g., Cao et al., 2006), are combined, thus reflecting a localized appearance of the BBFs).

A recent statistical study by Ohtani et al. (2004) emphasized the measurements made in the middle of relatively thick plasma sheet, and clearly reveals the time scale in a superposed epoch analysis (Fig. 2). Note the $\sim 1$ min duration of the magnetic $B_{z}$ bipolar variation, and the $\sim 2 \mathrm{~min}$ duration of the flow burst. It should be noted that the latter feature may not be as clear if the total flow is plotted instead of perpendicular flow. Similar behavior was also obtained for tailward flows (Fig. 8 of Ohtani et al., 2004). In rare cases such 1 min scale phenomena can be seen in isolation (e.g., the magnetic impulses studied by Sergeev et al., 2001). This time scale can be compared with the average $28 \mathrm{~s}$ duration of the core of the Earthward moving flux ropes (Slavin et al., 2003a) and with the average $35 \mathrm{~s}$ time scale of TCRs (Slavin et al., 2005), indicating a relationship among these phenomena (see also Sects. 2.3, 2.2 and Fig. 9). Moreover, the 1-3 min repetition rate of these impulsive events was previously identified as a basic time scale of processes related to the expansion phase of substorms, including the poleward auroral expansion, energetic particle injection to geostationary orbit, Pi2 pulsations, etc. (Sergeev et al., 1992).

Another temporal scale of interest is the lifetime of the fast plasma stream itself (until their interaction with the inner magnetosphere), which can be estimated from observations of BBF-related optical structures, viz. the lifetime of auroral streamers. This is typically several minutes (Sergeev, 2004). Thus, the temporal scales of BBFs include $\sim 1$ min scale of individual bursts, 5-20 min total duration for groups of such bursts, as well as the lifetime of individual plasma streams in the plasma sheet, which are also $\leq 10 \mathrm{~min}$.

The spatial scales of fast flows have not been measured directly, but can be estimated using the time scales discussed above. For example, with a flow speed $V=400 \mathrm{~km} / \mathrm{s}$, the 


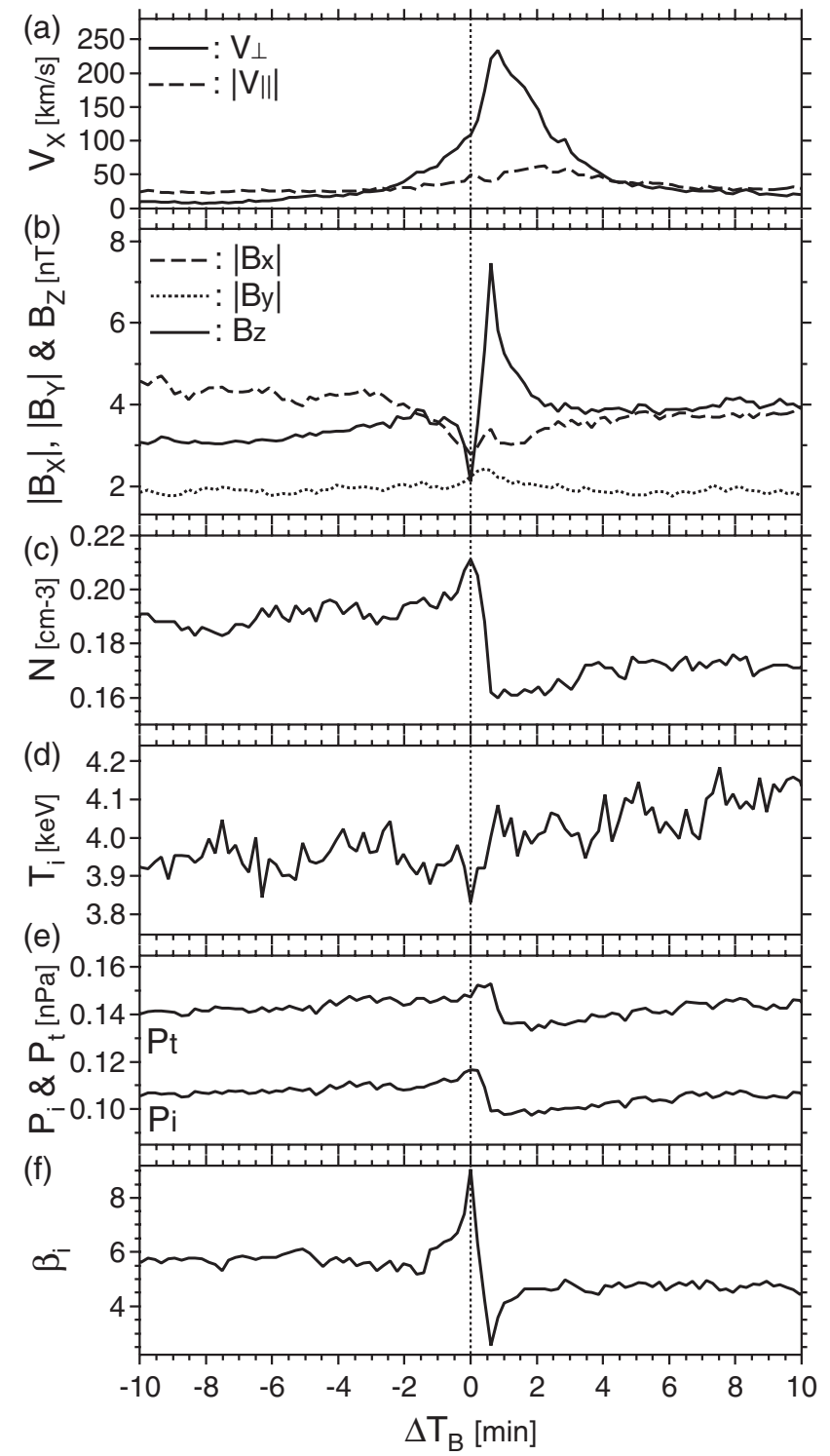

Fig. 2. The magnetic field and ion plasma parameters superposed for 818 fast Earthward flow events observed in the CPS by Geotail spacecraft with the start of a $B_{z}$ increase used as the zero time (from Ohtani et al., 2004).

1 min pulse and 10 min durations of the average BBF imply the lengths of $4 R_{E}$ and $40 R_{E}$, respectively. However the BBFs have a characteristic cross-tail scale of $Y \sim 2-4 R_{E}$ across the tail (corresponding to $\sim 1 \mathrm{~h}$ MLT in the ionosphere), which was consistently reproduced using different indirect methods (Sergeev et al., 1996; Angelopoulos et al., 1997; Nakamura et al., 2001a; Sergeev, 2004) and was also obtained in the recent statistical study of the flow gradients observed by Cluster (Nakamura et al., 2004a). Therefore, using the BBFs $3 R_{E} Y$-scale and $3 \mathrm{mV} / \mathrm{m}$ flux transport rate, the total flux transport (potential drop) across one narrow stream can be $\sim 60 \mathrm{kV}$. Since the potential drop required to close the global plasma circulation in the magnetosphere is $\sim 60 \mathrm{kV}$ on average, the BBFs are apparently able to provide nearly all the required plasma sheet transport. The observations of auroral streamers indicate a possibility of multiple individual plasma streams developing at the same time in different parts of the tail, as well as a possibility of complicated substructure inside particular streams (Kauristie et al., 2003; Sergeev, 2004).

Figure 2 provides a representative illustration of average behavior observed during the passage of isolated fast streams through the relatively thick and stable current sheet, consistent with the statistics presented in previous studies (e.g., Angelopoulos et al., 1997; Kauristie et al., 2000; Nakamura et al., 2001a).

The main features of BBFs are:

1. Reduction in plasma pressure in the flow region, mainly due to density decrease. Temperature enhancements are often observed but do not compensate for the density drop. The other signatures of efficient energization (e.g., bi-directional anisotropy of thermal electrons, Sergeev et al., 2001) are sometimes observed.

2. Magnetic field increases (positive/negative $B_{z}$ in Earthward/tailward flows, respectively) with magnetic pressure increase, partly compensating the plasma pressure drop. The BBF may be the underpopulated dipolarized flux tube (with magnetic flux closure across the current sheet consistent with the reconnection picture) or a bubble (see Sect. 3.6).

3. An asymmetric bipolar $B_{z}$ structure may be however evident in isolated BBFs with a brief (a few tens of seconds) opposite polarity pulse, with smaller amplitude preceding the main $B_{z}$ pulse.

4. Enhancements in the pressure and density, and a reduction in the plasma beta, before the sharp pressure and density drops, as shown in Fig. 2, may be associated with the interaction of the leading edge of the BBF with the surrounding media (Sergeev et al., 1996). The density recovery is more gradual and continues well after the flow subsides.

5. The above properties hold across the whole range (10$50 R_{E}$ ) covering the midtail and near-tail regions, although the peak velocity decreases significantly in the Earthward direction (from $>400 \mathrm{~km} / \mathrm{s}$ at large distances to $\leq 100 \mathrm{~km} / \mathrm{s}$ at $10 R_{E}$ if the BBF is defined using the flux transport criterion (Schödel et al., 2001, see also Fig. 5 in Ohtani et al., 2004b).

There are also indications that the passage of a localized plasma sheet bulge can be associated with the passage of an individual BBF pulse, and in these cases the observed characteristics have different interpretations (see Owen et al., 2005, and Sects. 2.4 and 2.3). 
The origin and the subsequent dynamics of BBFs are associated with two basic processes: local (temporal and spatial) features of reconnection (see Sects. 3.1, 3.3 and 3.2 for theoretical explanations) and interchange motions of the bubble (see Sect. 3.6). These two processes probably act together: Impulsive reconnection cuts the plasma tubes with smaller density (from outer PS or lobe tubes) providing the localized (in $X$ ) underpopulated plasma tubes carrying the fast flowing accelerated plasma. The interchange motion of these reconnected, underpopulated tubes allows them to penetrate deep into the closed plasma sheet region.

\subsection{Flux ropes and plasmoids}

Observations of bipolar variations in the $B_{z}$ component of the magnetic field, which is the component nominally in the normal direction to the cross-tail current sheet, have often been interpreted as the signature of a magnetic flux rope (although alternate interpretations are discussed in later subsections of this paper, see also Slavin et al., 1993). In this interpretation, the signature is caused by the motion of a magnetic flux rope structure, whose axis lies in the current sheet plane, moving past the spacecraft location.

A specific type of flux rope is that formed by closed loops of magnetic flux, which are termed plasmoids. These were first predicted by Hones (1977), based on a simple twodimensional cartoon model in which the occurrence of reconnection at a new, near-Earth neutral line (NENL) formed at, or near, substorm onset would result in the creation of closed loops of magnetic flux between the near- and distant neutral lines. The plasmoid would then be driven tailward at high speed by pressure gradients and/or the tension associated with field lines generated by subsequent reconnection of open lobe magnetic flux at the NENL. The theoretical background to flux rope and plasmoid formation in the magnetotail involves the occurrence of multiple active X-lines (or alternately a single X-line on closed field lines) which gives rise to the formation of magnetic loops or "islands" (Schindler, 1974). More details of this theory are discussed in Sect. 3.3. Many papers have reported observations of signatures consistent with the passage of these plasmoid-type structures in the distant magnetotail (e.g., Hones et al., 1984; Slavin et al., 1989; Moldwin and Hughes, 1992; Nagai et al., 1994; Slavin et al., 2002). These studies show, for example, that plasmoids can occur in a broad range of sizes, from $<10 R_{E}$ in the mid-tail $\left(-25>X_{\mathrm{GSM}}>-50 R_{E}\right)$, but increasing in size, on average, as they move downtail, where they are observed to have typical sizes of several tens of Earth radii beyond $X=-100 R_{E}$ (e.g., Ieda et al., 1998, 2001; Slavin et al., 1998, 1999). Their temporal duration was shown to be $<5$ min (Belehaki and Tsagouri, 2001).

The formation of completely closed loops of magnetic flux, as in the simple two-dimensional cartoon models of plasmoids, is in reality unlikely in the magnetotail. More generally, the magnetic fields of the two tail lobes are not ex- actly anti-parallel, in particular if a prevailing $B_{y}$ component of the interplanetary magnetic field is imparted to the tail by the action of reconnection between sheared magnetic fields at the dayside magnetopause (e.g., Cowley, 1981). The presence of even a small $B_{y}$ component of the fields in the plasma sheet may lead to the generation of magnetic flux rope structures, rather than the closed loops of the simple plasmoid models (e.g., Hughes and Sibeck, 1987; Birn et al., 1989; Moldwin and Hughes, 1991; Frank et al., 1994). The polarity of the flux ropes' core magnetic field should thus be largely determined by the direction of the IMF $B_{y}$ (Hughes and Sibeck, 1987). The occurrence of magnetic flux ropes in the magnetotail was first reported by (Sibeck et al., 1984) and many subsequent studies (e.g., Moldwin and Hughes, 1992; Lepping et al., 1995; Slavin et al., 1995; Ieda et al., 1998) have demonstrated that "plasmoids" in the mid- to distant tail do indeed have more helical magnetic field topologies. A review of distant tail plasmoid-type flux ropes and issues relating to their formation may also be found in Hesse and Kivelson (1998).

In addition to the tailward-moving plasmoids, there have been reports of individual flux ropes in the nearer-Earth plasma sheet being transported Earthward in the ISEE 1, 2 and 3, IMP 8 and the Galileo Earth flyby datasets (Elphic et al., 1986; Moldwin and Hughes, 1992; Sergeev et al., 1992; Kivelson et al., 1993; Moldwin and Hughes, 1994; Khurana et al., 1995). More recently, Slavin et al. (2003b) examined the Geotail measurements in the midtail and report the regular occurrence ( 1 every $\sim 5$ hours on average) of small ( $\sim 1-5 R_{E}$ diameter) flux ropes at $-20>X_{\mathrm{GSM}}>-30 R_{E}$. These are associated with high speed plasma sheet flows and are predominantly found near the leading edges of Earthward or tailward flow events. The terminology of either bursty bulk flow (BBF)-type flux ropes, for those associated with Earthward flow, or plasmoid-type flux ropes, when the structure is observed with tailward high speed flows was adopted by Slavin et al. (2003b). At these distances, approximately equal numbers of each type are observed. This is in contrast to the distant tail observations $\left(X_{\mathrm{GSM}}<-100 R_{E}\right)$, where $<10 \%$ of flux ropes are observed to move Earthward (e.g., Moldwin and Hughes, 1992).

The simplest representation of a flux rope is known as the "constant-alpha" force-free flux rope model (e.g., Chandresakhar and Woltjer, 1958; Taylor, 1986; Burlaga, 1988; Lepping et al., 1990). This model represents the minimum energy state for helical magnetic field lines, in which the current density $\mathbf{j}=\alpha \mathbf{B}$. It should be noted that this representation is strictly relevant to low-beta plasmas, although for simplicity it has been regularly applied to structures observed within the high-beta plasma sheet. Thus, using this model, the gross, large-scale properties of magnetotail flux ropes have been studied with the use of single spacecraft techniques (e.g., Elphic et al., 1986; Moldwin and Hughes, 1991; Slavin et al., 1995, 2003b). The topology of the magnetic field is a nested set of helical magnetic field lines ranging from a relatively 


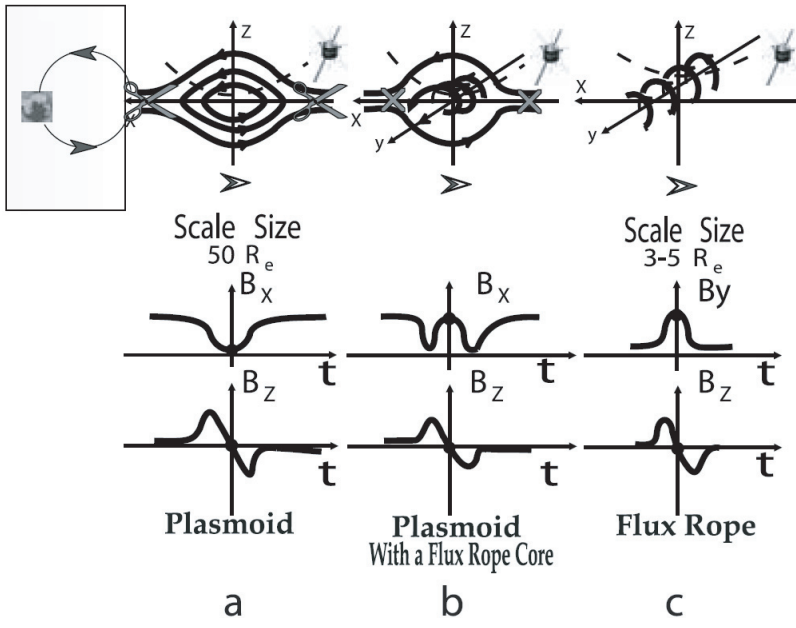

Fig. 3. Sketch of the magnetic configurations and signatures expected for (a) closed-loop plasmoid structures; (b) high-field regions (plasmoids with flux rope core) and (c) flux ropes. The spacecraft trajectory relative to the structure is shown as the dashed line in the upper panels, and leads to the signature shown below. The typical scale sizes in the distant tail are also indicated. (Adapted from Zong et al., 2004).

weak tangential field on the outer boundary of the flux rope to a strong core axial field in the centre. At all places in the rope the magnetic force, $\mathbf{j} \times \mathbf{B}=0$, since the current vector is everywhere parallel to the magnetic field vector. Evidence for the existence of tailward moving plasmoids with apparent forcefree flux rope topologies in the distant tail $\left(X<-230 R_{E}\right)$ has been presented (e.g., Moldwin and Hughes, 1991). In addition, Slavin et al. (2003b) reported many $\sim 2$ to $5 R_{E}$ diameter flux ropes between $-15<X_{\mathrm{GSM}}<-30 R_{E}$ were in a near-force-free state. Such observations could therefore represent well-developed, fully-evolved flux ropes which have relaxed, or nearly relaxed to a minimum energy state.

Flux rope structures often show a large increase in the magnitude of $B$ at the centre of the bipolar $B_{z}$ variation (Slavin et al., 1995). These are consistent with the flux rope having a strong core magnetic field, often significantly in excess of that in the exterior lobes, suggesting that these are not in pressure balance with the rest of the magnetotail. These observations of High Field Regions (HFRs), which typical have scale sizes of $\sim 5$ to $10 R_{E}$, led the authors to suggest that a force-free core may often be embedded within a larger non-force-free plasmoid. A summary sketch of the signatures of plasmoids, HFRs and flux ropes, together with their expected signatures is shown in Fig. 3 (adapted from Zong et al., 2004). An example of Geotail observations of a flux rope exhibiting a strong core field is shown in Fig. 4 (from Slavin et al., 2003b). Note the strong bipolar signature in the $B_{z}$ component of the field, lasting some $30 \mathrm{~s}$, and the very strong central core field which is largely in the $B_{y}$ component. The negative-positive sense of the variation in $B_{z}$ sug-

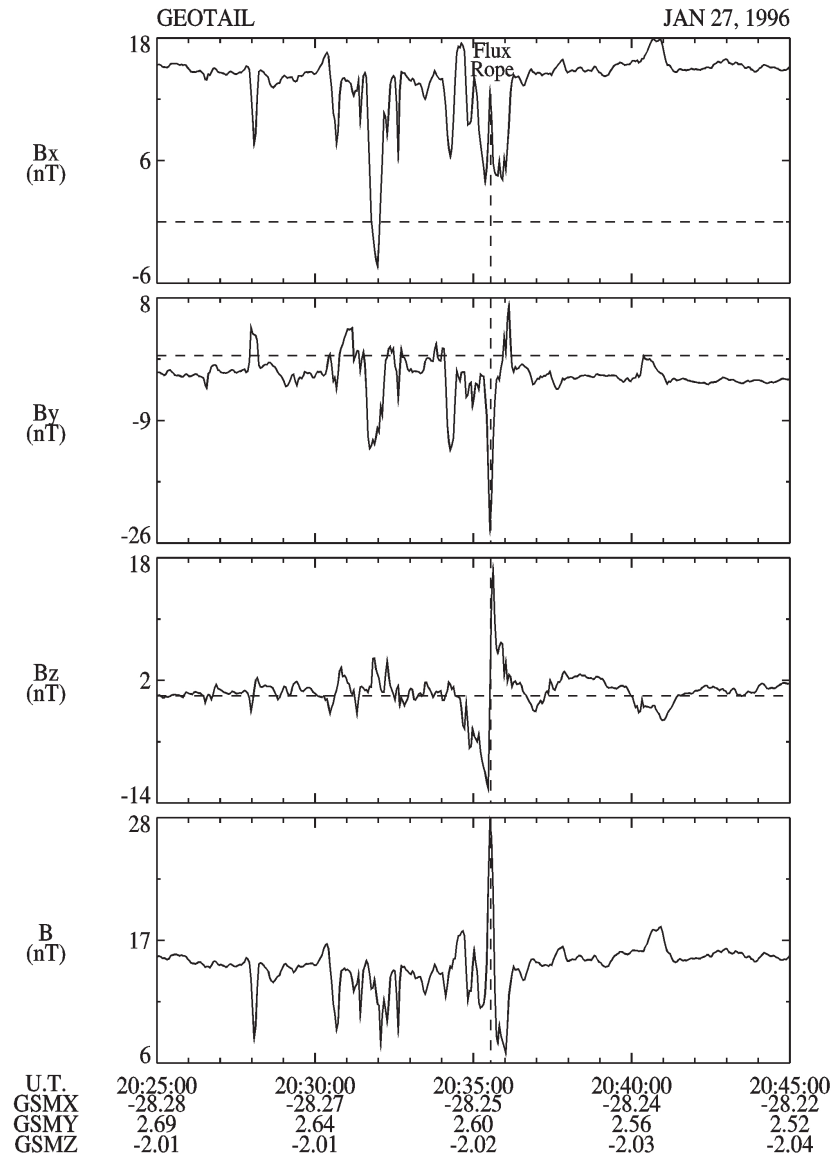

Fig. 4. Geotail observations of a flux rope with a strong core field (HFR) on 27 January 1996. The 3 GSM components of the magnetic field and its magnitude are presented. Note the negative positive bipolar signature in the $B_{z}$ component, and the central field magnitude enhancement greatly in excess of the background levels. This observation is thus consistent with a flux rope structure which contains a central force-free field region. (From Slavin et al., 2003b).

gests this is a BBF-type flux rope moving in the Earthward direction. Most such flux ropes are embedded in high-speed flows and have average diameters and core field intensities of $1.4 R_{E}$ and $20 \mathrm{nT}$ and $4.4 R_{E}$ and $14 \mathrm{nT}$ for the BBF- and plasmoid-type events, respectively. Flux ropes are usually not significantly inclined to the GSM $X-Y$ plane, but exhibit a wide range of azimuthal orientations within that plane.

Geotail observations were used to further identify internal plasma structures of flux ropes and plasmoids, as well as their temporal/spatial evolution, or relaxation, in the course of their downtail motion. The average energy carried by each plasmoid in the distant tail is estimated to be $(2.4 \pm 1.0) \times 10^{14} \mathrm{~J}$ (Belehaki and Tsagouri, 2001). Whereas the thermal energy flux dominates for the closed loop plasmoids, the kinetic energy was found to be comparable to or greater than the thermal energy flux in the flux-rope-type plasmoids. Ion composition measurements have shown that 
ion species from the solar wind and the ionosphere appear to be thoroughly mixed inside flux ropes or plasmoids, in a manner similar to the ion composition in the tail outside these structures (e.g., Wilken et al., 1995; Lui et al., 1998). Zong et al. (1997) reported quasi-periodic energetic ion bursts in the deep tail $\left(X_{\mathrm{GSM}}=-96 R_{E}\right)$ associated with plasmoid-like structures with bipolar signature in the $B_{z}$-component and in the $B_{y}$-component. Mukai et al. (1996) reported details of the counter-streaming ions and electron distributions found within a plasmoid and at its boundary. Frank et al. (1995) directly determined the field-aligned current densities using the plasma measurement in the core of a magnetotail flux rope with a cross-sectional size of $0.7 R_{E}$, at $X_{\mathrm{GSM}} \sim 96 R_{E}$. The total current was found to be 0.5 MA. Slavin et al. (1998) presented examples in which TCRs observed by IMP 8 at $X_{\mathrm{GSM}} \sim 40 R_{E}$ were subsequently followed by a plasmoid observation by Geotail at $X_{\mathrm{GSM}}=-170$ to $-197 R_{E}$. These unique two-point measurements clearly show that plasmoid ejection occurs near substorm onset, that they move rapidly into the distant tail and that they evolve and accelerate as they move tailward and perhaps encounter pre-existing $X$ lines in the distant tail.

More recent studies of magnetic flux ropes in the tail have been undertaken using the 4 point Cluster observations. Since Cluster apogee is in the relatively near-tail, $X_{\mathrm{GSM}} \sim-19 R_{E}$, these results are necessarily more restricted than those from more extended excursions into the tail. Nevertheless, many of the properties of flux ropes in this region have been investigated with Cluster (e.g., Slavin et al., 2003a; Zong et al., 2004; Eastwood et al., 2005; Henderson et al., 2006a). These results confirm, using multi-spacecraft timing techniques, that events with a south-then-north (north-thensouth) signature indeed move Earthward (tailward), and are usually embedded in fast plasma flows (Slavin et al., 2003b). Furthermore, Cluster observations of traveling compression regions (Slavin et al., 2005), a probable signature of the passage of flux ropes in the lobes (see Sect. 2.3), show that at very near-tail distances (i.e. $X>-20 R_{E}$ ) $80 \%$ of flux ropes travel Earthward.

Slavin et al. (2003a) reported on a flux rope observed by Cluster in the central plasma sheet, where all four spacecraft sampled the structure directly. Multi-spacecraft timeof-flight techniques were used to show that the flux rope was moving Earthward at a speed $\sim 700 \mathrm{~km} / \mathrm{s}$, in close agreement to the directly-measured plasma velocities in the associated bursty bulk flow. Based on the duration of the signature, the cross-sectional size of the structure was $\sim 1 R_{E}$. Note that these structures are thus much smaller than their counterparts observed in the mid- and distant tail. This may be a result of the expansion of such structures as they move tailwards, or more likely reflects the scale size of the formation process. For example, distant tail plasmoids may be formed between a pre-existing reconnection neutral line and a new, substormassociated neutral line separated by several tens of $R_{E}$. Conversely, the flux ropes at Cluster apogee may be the result of break-up of the near-Earth current sheet with separations between reconnection sites, and hence flux rope sizes, of only a few $R_{E}$. The possible role of multiple X-lines in current sheet dynamics is discussed in Sect. 3.3.

Using the ability of Cluster to determine the current density with the curlometer technique (e.g., Dunlop and Woodward, 2000), Slavin et al. (2003a) also showed that the peak current density was $17 \mathrm{nA} / \mathrm{m}^{2}$ near the central axis of the rope. However, the perpendicular currents were larger than the parallel currents for the first half of the rope encounter, so this rope was not in a force-free configuration. However, Slavin et al. (2003a) also noted a general agreement of the field profiles at each spacecraft with the magnetic field topology of the force-free type. These models return a prediction for the peak electric currents along the central axis of the flux rope in the range $15-19 \mathrm{nA} / \mathrm{m}^{2}$. Hence, while the single-spacecraft models may be successful in fitting flux rope magnetic field and current variations, they do not provide a stringent test of the force-free condition.

Eastwood et al. (2005) presented a Cluster multispacecraft analysis to demonstrate that the variations in field and flow observed in the vicinity of the magnetotail current sheet are consistent with a series of two active reconnection sites bounding an Earthward-moving flux rope. These authors point out that single spacecraft analysis of the same data could lead to the incorrect conclusion that a single Xline is moving tailward past the spacecraft. This provides a demonstration of the power of the Cluster multi-spacecraft measurements in informing our understanding of magnetotail dynamics.

Henderson et al. (2006a) have also examined the structure of flux ropes in the near-tail with the Cluster observations. They report a dearth of examples of flux ropes with a strong core field at these distances, with most tending to be magnetic loop structures either completely without, or with only small core fields. They presented an example of a flux rope observed at $X_{\mathrm{GSM}} \sim-17 R_{E}$ during the 2003 season (in which Cluster inter-spacecraft separations were smallest, at several $100 \mathrm{~km}$ ). Multi-spacecraft analysis revealed the flux rope to be small in size, $\sim 0.3 R_{E}$, and moving Earthward and duskward at $\sim 160 \mathrm{~km} / \mathrm{s}$. The current inside the flux rope, determined from the curlometer technique, was predominantly parallel to the magnetic field, but in the outer sections, $\mathbf{j} \times \mathbf{B}$ was significant, and directed such to suggest that the magnetic pressure force was acting to expand the flux rope at the time of observation. However, the electron pressure, determined from the PEACE instruments, was reduced inside the flux rope. A second example, reproduced here in Fig. 5, was, in contrast, a tailward-moving flux rope, but showed similar results. These observations of large magnetic forces in the outer sections of the flux ropes compared to the centre may be explained if the flux ropes are observed at an intermediate stage of their relaxation after creation by reconnection at multiple X lines (see Sect. 3.3) near the Cluster apogee. The outer parts of flux ropes may thus be at a relatively early 


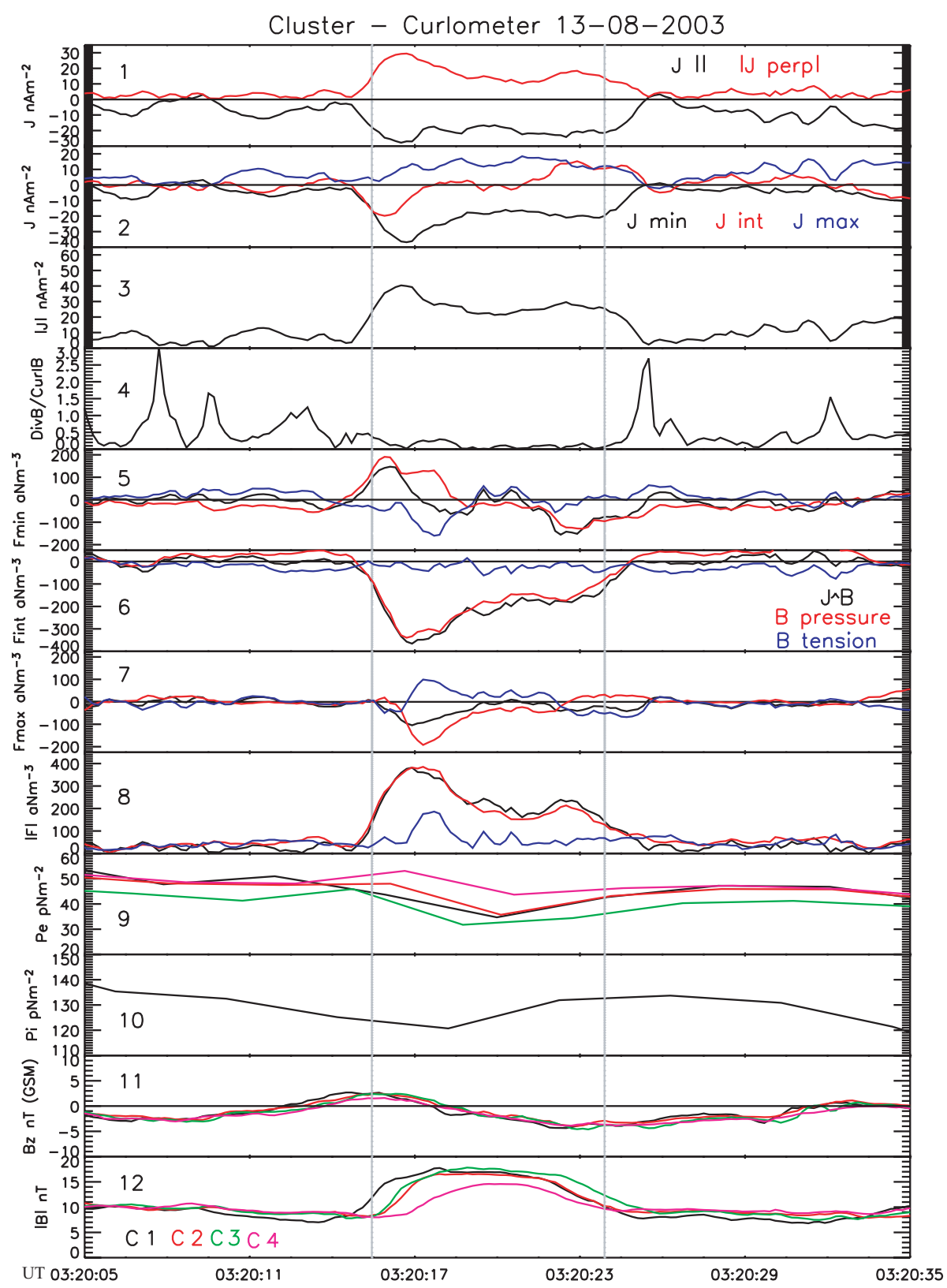

Fig. 5. Cluster observations of a small-scale, tailward-moving flux rope on 13 August 2003. Panel 1 shows the current parallel (black) and the magnitude of the current perpendicular (red) to the magnetic field. Panel 2 shows the current projected into the minimum (black), intermediate (red) and maximum (blue) variance directions, respectively. Panels 3 and 4 show the magnitude of the current and the fractional error in the curlometer result, respectively. Panels 5 to 7 show $\mathbf{J} \times \mathbf{B}$ (black), the magnetic pressure (red) and the magnetic tension (blue) projected onto the minimum, intermediate and maximum variance directions of Cluster 1, respectively. Panel 8 shows $|\mathbf{J} \times \mathbf{B}|$ and the magnitudes of the magnetic pressure and tension forces. Panels 9 and 10 show the electron and ion pressure, respectively, with the final two panels showing the $B_{z}$ and $|B|$ observations illustrating the bipolar signature and increase in $|B|$, respectively. The observations illustrate significant magnetic forces within the flux rope, especially towards the edges, and support the premise that the structure has yet to evolve into the force-free state associated with flux ropes in the more distant tail (from Henderson et al., 2006a).

stage of the process of relaxing towards the force-free configuration which is often observed in such structures further down the tail. However, the centre of the flux ropes may contain older reconnected flux at a later evolutionary stage and may therefore be more force-free.
Zong et al. (2004) showed that energetic electrons inside plasmoid-like structures are confined within a smaller spatial region than the energetic ions, which represent a good indicator for the structure boundaries. The ion composition observed inside the plasmoid showed significantly lower $\mathrm{O}^{+}$ 
and $\mathrm{He}^{+}$than in the ambient plasma. This implies few heavy ions are involved in the reconnection process where the plasmoid is formed. Multiple flux rope/plasmoid observations presented in this paper were interpreted as strong evidence for multiple X- lines. Note the nature of transient energetic particle signatures in the magnetotail is discussed in further detail in Sect. 2.10.

Xiao et al. (2004) re-examined the accuracy of flux rope orientations inferred from both the traditional magnetic fieldbased minimum variance analysis (BMVA) technique and the current-based MVA (CMVA) approaches, and compared these to the techniques which have become deployable since the advent of Cluster multi-point observations. They found that the directions of eigenvectors of MVA are critically dependent on the spacecraft path relative to the flux rope axis and the model of the flux rope used. For force-free flux ropes, the $\mathrm{M}$ direction of BMVA best fits the axial orientation, while for non-force-free flux ropes the BMVA may fail as a tool for determining the orientation of flux ropes. Magnetic field data from a single satellite path through non-force-free flux ropes are often insufficient to determine the rope orientation, since none of the returned eigenvectors necessarily correspond to the flux rope axis. However, it was shown that the CMVA test, which is based on the multiple spacecraft measurements, may help to eliminate this uncertainty and may thus be more useful for the study of structures and geometries of observed flux ropes.

\subsection{Traveling compression regions}

Traveling compression regions (TCRs) in the distant tail, $X<-30 R_{E}$, are several minute long, compressive, $\triangle B / B \sim 5-10 \%$, perturbations of the lobe magnetic field. For definiteness, in this section, it is assumed, along with many authors, that the passage of a plasmoid/flux rope is the primary cause of TCRs in the tail, that produces local increases in the thickness of the plasma sheet and thus compresses the surrounding lobe regions. The validity of this assumption is addressed at the end of this section. Figure 6 provides a schematic diagram of how the lobe magnetic fields are compressed and drape about a large plasmoid-type flux rope as it is ejected down the tail (Slavin et al., 1984).

These compressions are standing features in the frame of the flux rope, but they "travel" in the spacecraft frame as the underlying flux rope moves either Earthward or tailward. The characteristic signature, a north-then-south (NS) tilting of the lobe field centered upon a smooth compression, was first noted in the Explorer 35 observations in the mid-1970s (Maezawa, 1975). However, the first definitive studies of this phenomenon and the discovery of the underlying plasmoidtype flux ropes awaited the re-direction of ISEE 3 into the distant magnetotail in 1982-1983 (Hones et al., 1984). Since that time, TCRs have been studied extensively using observations from ISEE 3 (e.g., Moldwin and Hughes, 1992; Slavin et al., 1993), IMP 8 (e.g., Taguchi et al., 1998b), Galileo

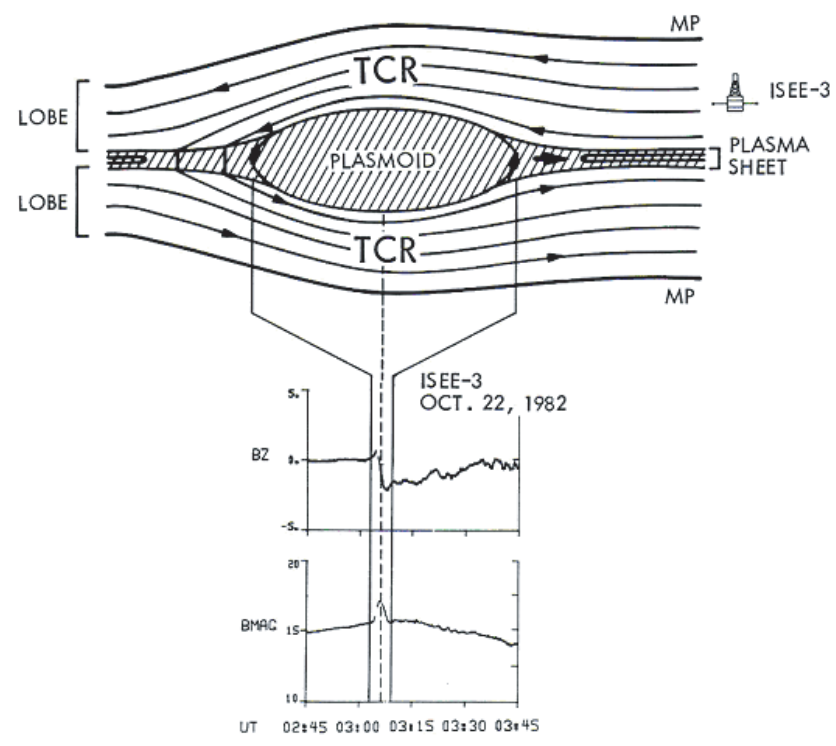

Fig. 6. A schematic view of a traveling compression region is presented along with actual magnetic field magnitude and $B_{z}$ observed by ISEE 3 during a TCR on 22 October 1982. (Adapted from Slavin et al., 1984).

(Kivelson et al., 1993) and GEOTAIL (e.g., Nagai et al., 1994; Kawano et al., 1994; Shirai et al., 2001).

TCRs have been used to infer the number and size of flux ropes being carried predominantly tailward by fast plasma sheet flows (Slavin et al., 1993), to relate energetic particle acceleration to specific reconnection events (Murphy et al., 1987; Owen and Slavin, 1992; Richardson et al., 1996), and to time the progression of substorms from the onset of fast reconnection (Slavin et al., 1992; Moldwin and Hughes, 1992; Taguchi et al., 1998a; Slavin et al., 2002; Huang et al., 2003). In this manner, it has been determined that the vast majority of all TCRs observed beyond $X \sim-30 R_{E}$ are tailwardmoving and closely related to the onset of substorm activity. Note also that 'unipolar' TCR signatures have been reported in the mid-tail, notably by Taguchi et al. (1998a,b) using IMP-8 data near $X_{\mathrm{GSE}} \sim 40 R_{E}$. The explanation for these signatures is the formation of the TCR (and thus underlying flux rope) at these distances, prior to ejection and movement downtail. In this scenario, the spacecraft detects only the second half of the otherwise bipolar $B_{z}$ signature.

Slavin et al. (2003c), Borälv et al. (2005), Owen et al. (2005), and Sergeev et al. (2005) have reported the first observations of TCRs in the lobes of the near tail using the Cluster measurements. While the amplitudes of the Cluster TCRs were similar to those measured further down the tail, the duration of these perturbations were typically only a few tens of seconds, as contrasted with $\sim 1-3$ min in the ISEE 3 observations (Slavin et al., 1993), consistent with the different scale sizes of the undelying plasmoid/flux ropes in these regions, as discussed in Sect. 2.2. Slavin et al. (2003c, 2005) 


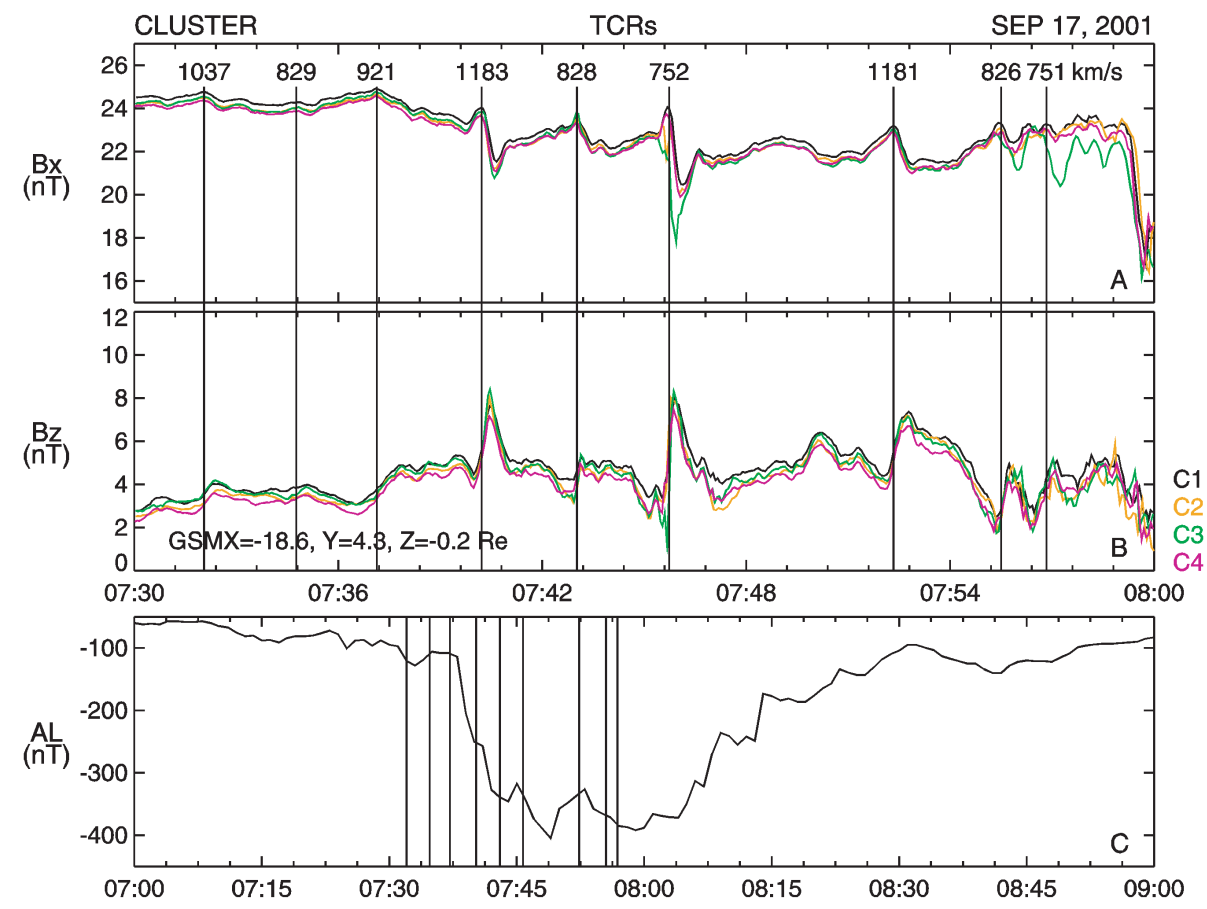

Fig. 7. Example of TCRs observed by Cluster during a substorm on 17 September 2001. The propagation speed of the TCRs, all Earthward, are shown in the top panel. (From Slavin et al., 2005).

found that these brief compressions of the lobe field were accompanied by the familiar $B_{z}$ perturbations observed in the distant tail, but in this region approximately $80 \%$ were observed to exhibit a $-/+B_{z}$, or south-then-north $(\mathrm{SN})$ variation. These TCRs can still be explained in the manner shown in Fig. 6, but the motion of the compression and underlying flux rope must be Earthward. Fortunately, the four spacecraft Cluster mission allows the direction of propagation of the TCRs to be determined directly. In all cases the arrival times of the perturbation at the different Cluster spacecraft showed that NS and SN TCRs moved tailward and Earthward, respectively.

An example of near-tail TCRs observed during a welldefined substorm on 17 September 2001 is displayed in Fig. 7. Nine TCRs are marked with vertical lines. Each TCR in this example exhibit the $\mathrm{SN} B_{z}$ variation indicative of Earthward propagation. Their motion is confirmed using the times of arrival of the perturbation at the different Cluster spacecraft. The Earthward propagation speeds determined by Slavin et al. (2005) are shown in the top panel above each of the TCRs. Their speeds, which range between $\sim 750$ to $1200 \mathrm{~km} / \mathrm{s}$, are comparable to the observed flow speeds measured for bursty bulk flows (BBFs) in the plasma sheet (e.g., Nagai et al., 2000; Angelopoulos et al., 1992; Baumjohann et al., 1990). The bottom panel of Fig. 7 displays the Kyoto Quick-Look AL index. Following a slow ( $30 \mathrm{~min})$ decrease, sometimes attributed to a "growth phase", there is a rapid onset of an expansion phase at 07:38 UT and a broad negative bay between 07:48 and 08:00 UT. The recovery to pre-onset levels then takes about $1 \mathrm{~h}$. The times of the TCR signatures denoted in the upper panels are marked by the vertical lines (note the extended timescale in this lower panel). The occurrence of TCRs beginning around substorm onset and continuing through the peak of the expansion phase, as shown in Fig. 7, is characteristic of the Cluster TCR population as a whole.

In contrast with the several minute separations between TCRs observed during the substorm in Fig. 7, Cluster also observes events with temporal separations close to the duration of the individual TCRs, i.e. $\sim 30$ to $60 \mathrm{~s}$. Figure 8 shows an example of such multiple, closely-spaced TCRs on 24 August 2001. The durations of the individual compression regions is $\sim 0.5$ to $1 \mathrm{~min}$ while the separations between the mid-points of the TCRs vary from $\sim 1$ to $2.5 \mathrm{~min}$. Interestingly, the amplitude of the first TCR is the largest and the compression ratios of the later ones are smaller, as is also commonly observed in the distant tail (Slavin et al., 1993).

A superposed epoch analysis comparing the TCRs observed by Cluster and the direct observations of magnetic flux ropes by Geotail is presented in Fig. 9 (Slavin et al., 2005). The window for the analyses is $+120 \mathrm{~s}$ and the zero epoch is taken to be the center of the TCR perturbation at s/c 1 and the inflection point in the flux rope $B_{z}$ variation, respectively. The $B_{x}$ and $B_{z}$ components of the Cluster magnetic fields are shown for the TCRs in the top two panels the figure. Similarly, the $\left|B_{y}\right|$ and $B_{z}$ components of the flux 


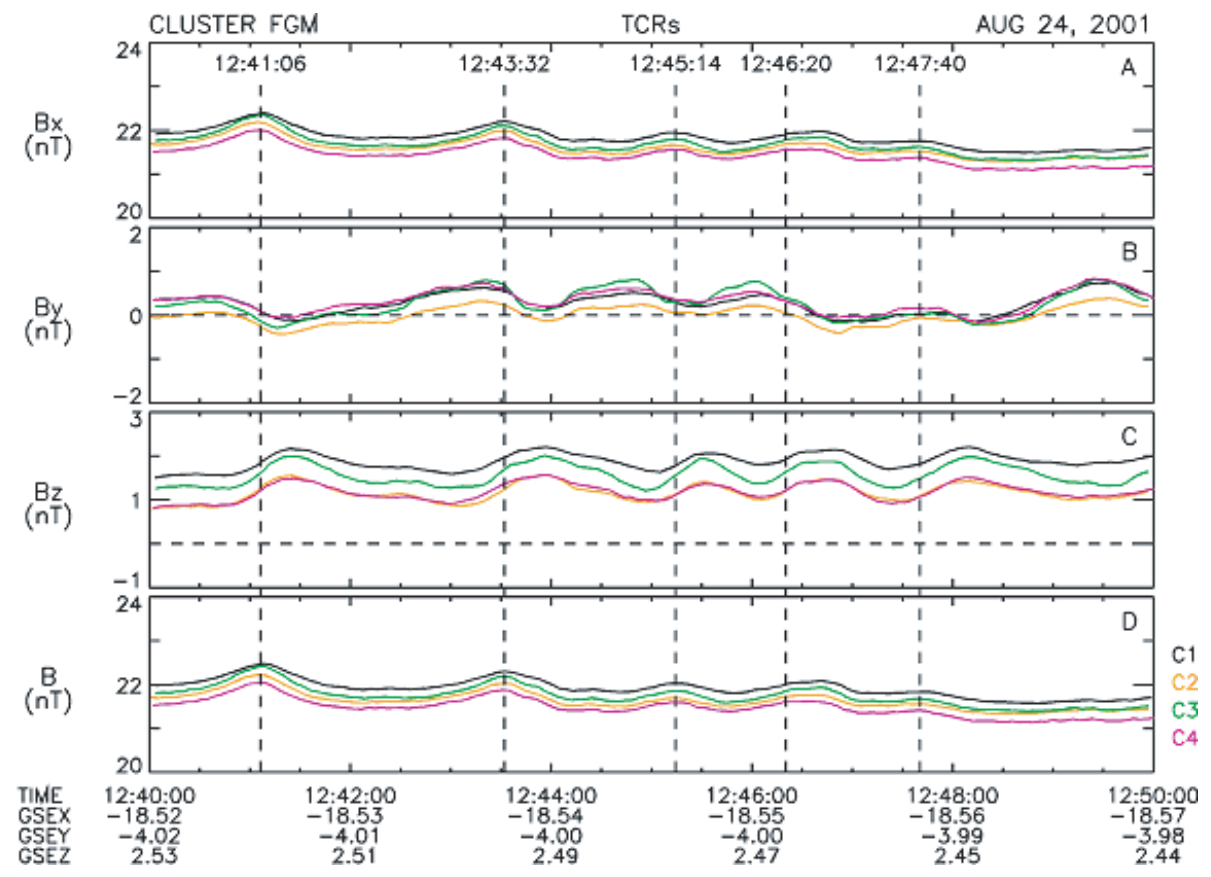

Fig. 8. Example of a multiple SN TCR event observed on 24 October 2001.

rope magnetic fields observed by Geotail are displayed in the middle two panels. Comparison of the traces shows that the duration of the near-tail TCRs and flux ropes are comparable at $\sim 30-40 \mathrm{~s}$. The fact that TCR perturbations are just slightly broader than the flux ropes is indeed consistent with this lobe field perturbation being due to the "draping" or "tenting" of the lobe flux tubes about a bulge in the plasma sheet. Close inspection of the magnetic field traces also shows that, for the Cluster TCRs, the superposed perturbation is first seen at s/c 4 and, a few seconds later, at s/c 1 . Since for this dataset s/c 1 and s/c 4 were located near the Sun-Earth line with separations in $X_{\mathrm{GSM}}$ of several thousand kilometers that place s/c 1 closer to Earth than s/c 4, this is consistent with an overall Earthward propagation of the TCR population within the study. Finally, the plasma sheet ion beta $\left(\beta=2 \mu_{0} n k T_{i} / B^{2}\right)$ and flow velocity are shown for the flux ropes confirming that they are high beta $(\beta \sim 10)$, central plasma sheet structures associated with BBF-type Earthward flows.

As shown in Fig. 6 and discussed above, the basic assumption of the TCR being the lobe signatures of a flux rope (Sect. 2.2) which is considered to be created as a consequence of multiple X-line (Sect. 3.3) thus apppears sound. This explanations has been confirmed and greatly refined using the results of numerical simulations (e.g., Ugai and Zheng, 2006a,b). These simulations also reproduced the observed progression of size in multiple TCR (Slavin et al., 1993).

\subsection{Nightside flux transfer events}

The term Nightside Flux Transfer Events (NFTEs) was introduced by Sergeev et al. (1992) to emphasize the impulsive nature and profound similarity between magnetopause FTEs and plasma sheet fast flows (BBFs). That is, it was recognized that in contrast to the 2-D tearing/plasmoid picture in the magnetotail, the BBFs involve characteristics of a 3-D propagation of localized reconnected flux tubes such as for the FTEs at dayside magnetopause. By observing the plasma sheet boundary at two ISEE spacecraft separated in $Z$, they emphasized the following properties of an individual flow pulse during an NFTE, shown schematically in Fig. 10:

1. The bipolar $B_{z}$ signature is often present, but is typically very asymmetric, suggesting that, unlike the plasmoid or flux rope structure, the flow burst predominantly carries the magnetic flux of one polarity (southward flux in the tailward flow, northward flux in the Earthward flow). Such asymmetric bipolar $B_{z}$ behavior is also seen in statistical patterns of both the BBFs (Fig. 2), and of flux ropes (Fig. 9) (note that $B_{z}$ stays positive in both). This suggests a (locally) open magnetic structure of the bulge in Fig. 10, which is inconsistent with a closed loop pattern of plasmoids or flux ropes.

2. When observed just outside the PS boundary (e.g. in Fig. 11, see also Fig. 7, Fig. 8), the localized bulgelike expansion of the plasma sheet traveling with the flow burst is evidenced by outward/inward vertical convective flows, which anti-correlate (in northern lobe, in 


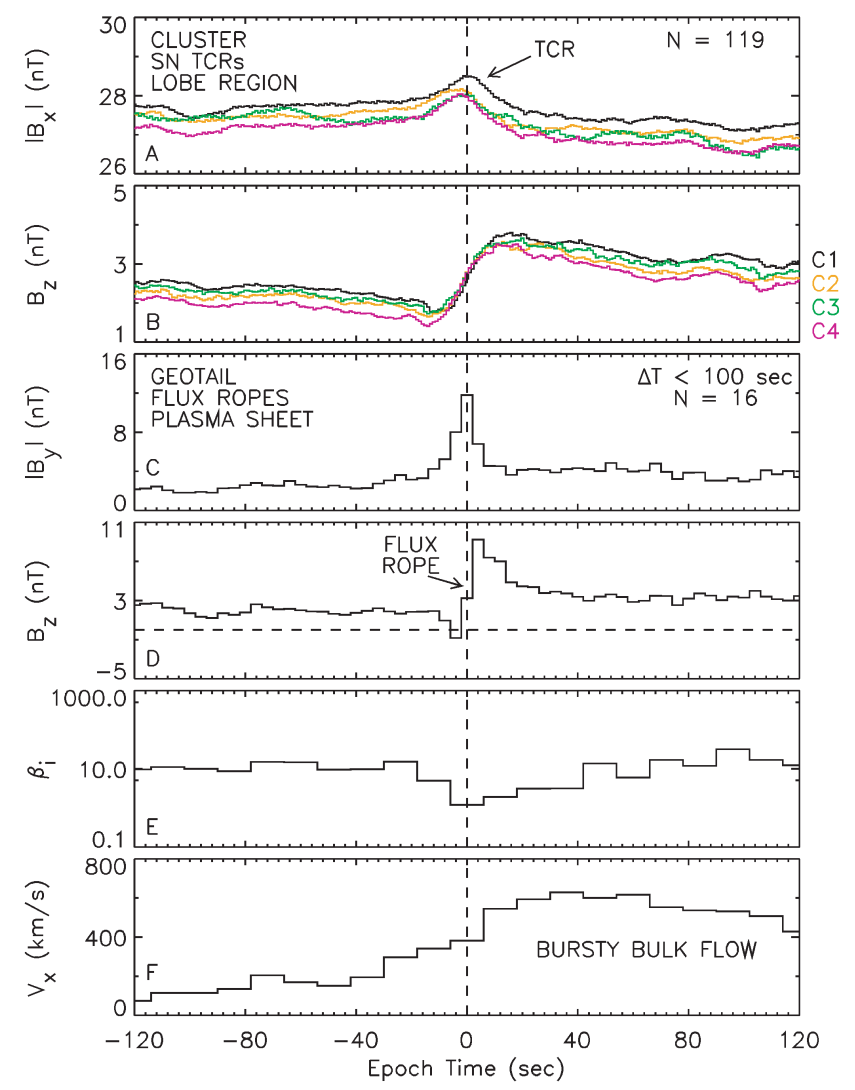

Fig. 9. Merged superposed epoch analyses of Cluster SN TCRs and Geotail BBF-type flux ropes. (From Slavin et al., 2005).

case of Earthward propagation) with $B_{z}$ variations. Another signature of a propagated plasma sheet bulge is the $B_{x}$-compression shifted in phase against $B_{z} / V_{z}$ (bipolar) variations. A feature noticeable in isolated events is that $B_{x}$ stays at a lower level after the bulge passage than prior to this event, suggesting a partial disruption of the tail current.

We emphasize that these signatures of NFTEs are basically similar to those of TCRs (Sect. 3.1) or current disruption (Sect. 2.6), and thus they may be members of the same family. The transient reconnection model (Sect. 3.1) provides a straightforward interpretation of the NFTE features described above.

\subsection{Transients in field aligned currents}

Field-aligned currents (FAC) are key to the magnetosphereionosphere coupling. Early FAC current observations (Elphic et al., 1985; Ohtani et al., 1988) in the midtail region were used to study the relationships to the Region 1 and Region 2 large-scale current systems established by low-altitude spacecraft observations (Iijima and Potemra, 1978). These studies obtained general, but not exactly one-to-one, agree-

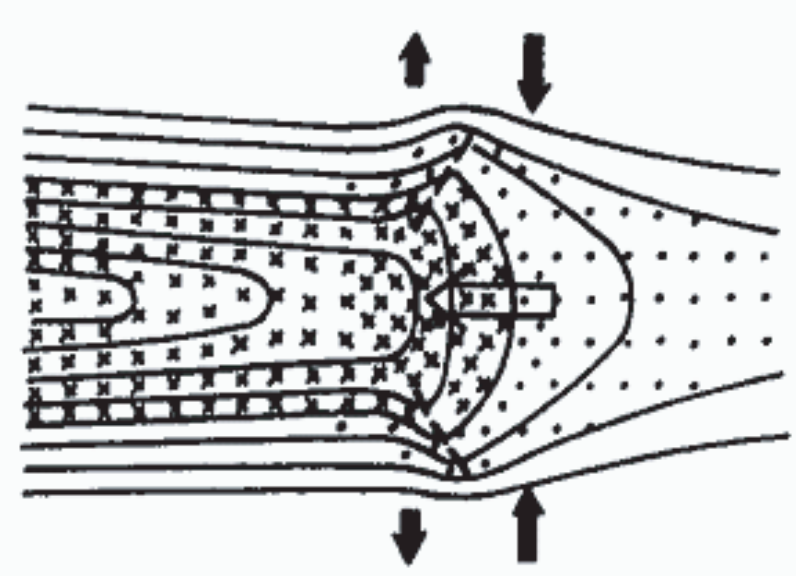

Fig. 10. Configuration of NFTE structure consistent with their observed signatures. (From Sergeev et al., 1992).

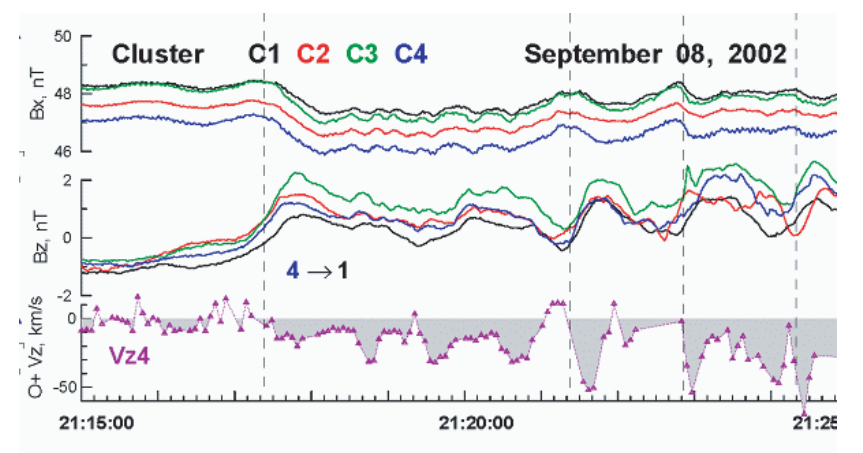

Fig. 11. Cluster observations near the plasma sheet outer boundary at substorm onset. Variations of the $B_{x}$ and $B_{z}$ components are shown together with vertical flow component of cold oxygen at spacecraft $C 4$. Note the anticorrelation between $B_{z}$ and $V_{z}$ variations and phase shifts between $B_{x}$ compressions (marked by vertical dashed lines) and $B_{z}$ variation. From cross-correlation these structures were found to propagate Earthward at $\sim 600 \mathrm{~km} / \mathrm{s}$. (From Sergeev et al., 2005).

ment, indicating different current systems should be taken into account.

FAC are conventionally observed as a magnetic field disturbance perpendicular to the main component, which corresponds to a disturbance of the $B_{y}$ component in the canonical tail configuration assuming FAC occur in sheets. This assumption of a sheet current, together with assumptions about the velocity of the structure relative to the spacecraft, are necessary to determine the current density using single spacecraft observations. However, some of the FAC are associated with localized and transient plasma signatures discussed in the previous sections $(2.1,2.4)$, which will be the focus of this section. Cluster has enabled the first determination of the spatial and temporal scales, as well as the current density 
of these transient FAC.

Transient FAC signatures associated with BBFs can be observed at the leading edge of the fast flowing plasma. Magnetic field and plasma properties around this region were first studied by Sergeev et al. (1996). They found that shear in the magnetic fields and flows between the leading edge region and the main body of the BBF produces a FAC pattern similar to a localized current wedge, i.e. FAC out of (into) the ionosphere at the dusk (dawn) section of the leading edge. Simultaneous observation between satellite and ground-based observations supported this current wedge pattern associated with BBFs (Kauristie et al., 2000; Grocott et al., 2004). The FAC region on the duskside of a bursty bulk flow, where the current is flowing out of the ionosphere, has been identified consistently with the electron precipitation region in auroral images (Nakamura et al., 2001b; Sergeev et al., 2004a).

Cluster multipoint observations by Nakamura et al. (2005) obtained the thickness of this shear region, which was about $2000 \mathrm{~km}$, and identified a consistent temporal/spatial scale for the FAC system in the conjugate ionosphere. For a BBF front at the center of the plasma sheet the magnetic shear appeared mainly in the $B_{y}$ or $B_{x}$ component (Sergeev et al., 1996). However, when the front moves in dawn-dusk direction relative to the spacecraft and the spacecraft enters the fast flow region from the side in an off-equatorial region, the FAC disturbance is expected to be observed in $B_{z}$ component which will provide seemingly similar temporal profile as Earthward/tailward moving flux rope (Sect. 2.2) or NFTE (Sect. 2.4). Snekvik et al. (2007) reported such localized FAC at the south-eastern edge of a fast flow, as shown in Fig. 12. Cluster encountered the fast flow region, where $V_{x}$ is close to $600 \mathrm{~km} / \mathrm{s}$ (Fig. 12b), in the outer plasma sheet, where $\beta$ was between 0.5 and 1 (Fig. 12a). At the leading edge of the fast flow, a negative to positive change of $B_{z}$ was observed (Fig. 12c). This $B_{z}$ perturbation was clearly associated with a FAC (Fig. 12d) flowing Earthward into the ionosphere (e) as indicated by the dashed vertical line in Fig. 12. Using different four-spacecraft analysis techniques, including the GS reconstruction technique (see Sect. 3.4 and Fig. 35), the orientation of the FAC and the detailed motion of the spacecraft relative to the plasma flow structures were determined. The thickness was found to be $2500 \mathrm{~km}$, which is a comparable to that determined by Nakamura et al. (2005). This was shown to be a localized structure relative to the entire flow. The total current was estimated to be about $0.1 \mathrm{MA}$, which corresponds to about $5-10 \%$ of the total current of the Region 1 current circuit. The outstanding difference between the $B_{z}$ reversal in Fig. 12 and those $B_{z}$ reversals associated with the conventional plasmoid/flux rope or nightside flux transfer event (Sects. 2.4 and 2.2) is that the former magnetic disturbance is propagating in a direction not aligned with the fast flow. That is, FAC-associated $B_{z}$ reversal was observed here due to the duskward motion of the spacecraft relative to the Earthward streaming plasma (Fig. 12f).

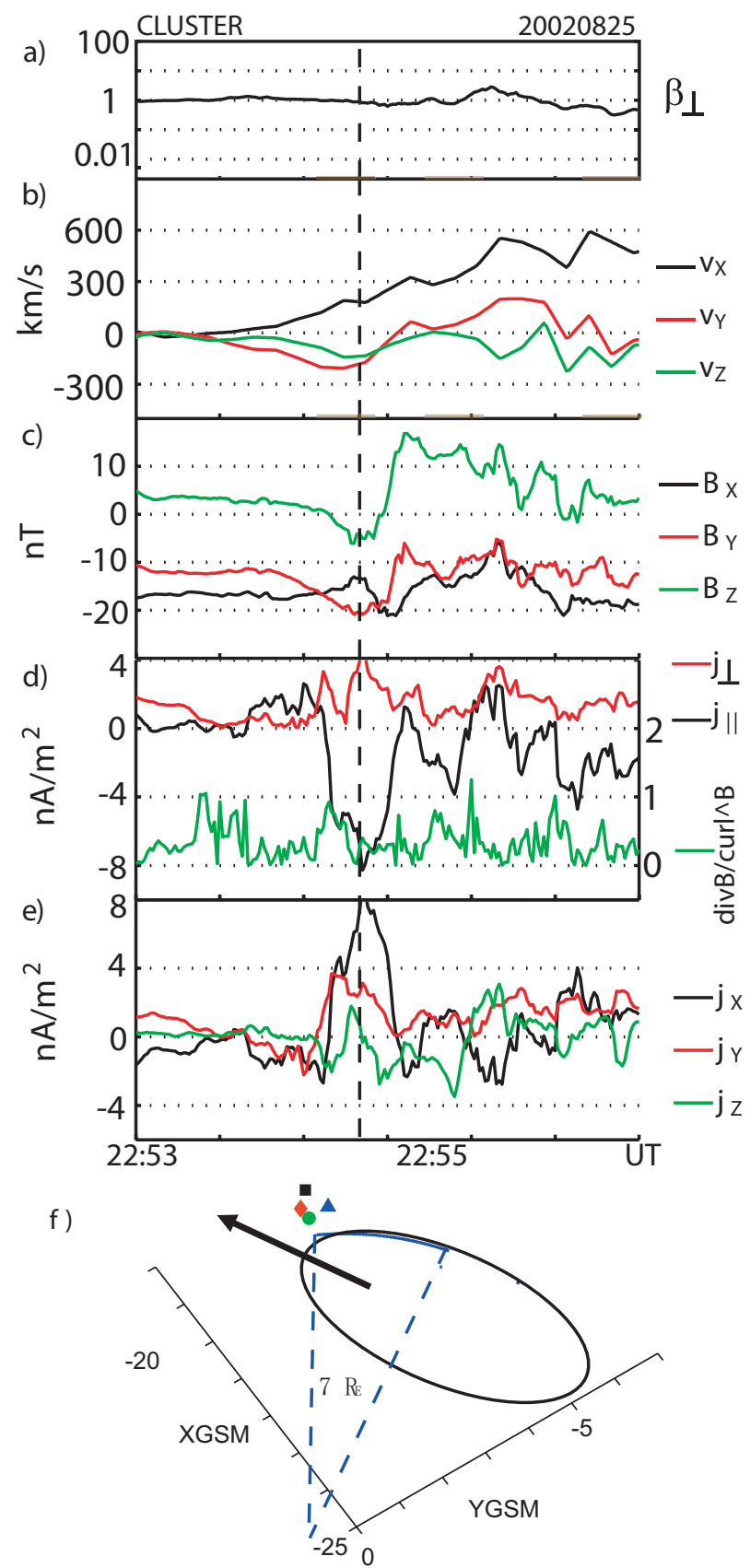

Fig. 12. Cluster measurements during a fast flow event on $25 \mathrm{Au}-$ gust 2002: (a) proton $\beta$, (b) proton velocity and (c) magnetic field obtained by Cluster 1, and (d) perpendicular and parallel components of curl of $B$ relative to the average magnetic fields and the ratio of curl of $\mathrm{B}$ to divergence of $\mathrm{B}$ and (e) $X, Y$, and $Z$ components of curl of B. (f) Schematic presentation of the BBF and the spacecraft entering the fast moving plasma from the dawn edge. (Adopted from Snekvik et al., 2007).

Another type of transient FAC was reported by Fujimoto et al. (2001), based on Geotail observations. This is a downward FAC (flowing into the ionosphere) carried by 


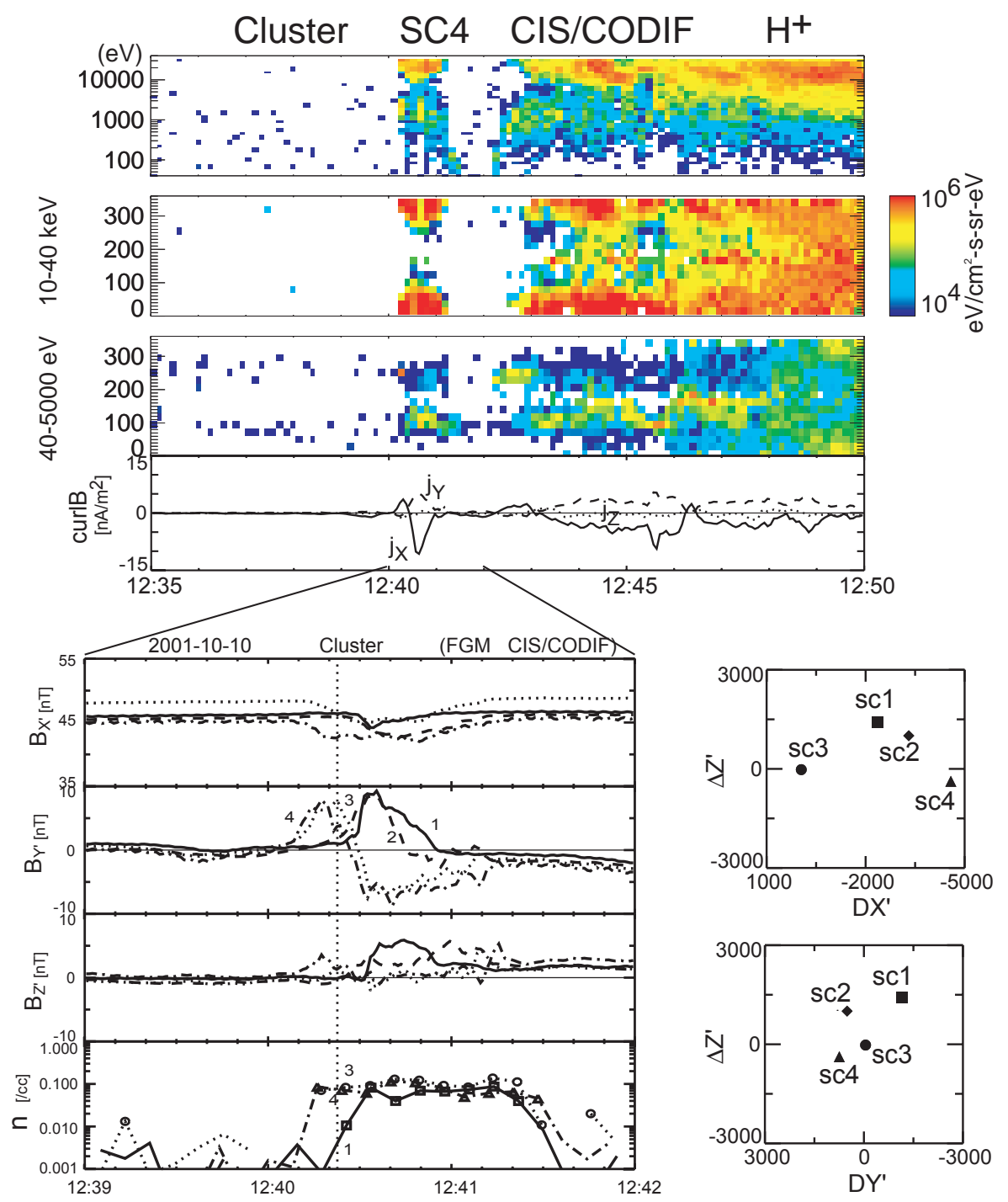

Fig. 13. Observation of a transient field-aligned current at the plasma sheet boundary layer. Proton energy spectra, pitch angle distribution of high energy $(10-40 \mathrm{keV})$ and low energy $(40 \mathrm{eV}-5 \mathrm{keV})$ protons for $\mathrm{C} 4$, and current density obtained from four spacecraft data are shown in the top panel. The relative location of the four spacecraft in the plasma sheet boundary coordinate system is given in the two panels at the lower right. Magnetic field from C1-C4 and proton density from C1 and C3 during the first plasma sheet encounter are shown in the lower left panels for the time interval when Cluster observed strong field-aligned currents. (Adopted from Nakamura et al., 2004b).

field-aligned tailward flowing electrons associated with energetic ion beams $(>10 \mathrm{keV})$ at the lobe-plasma sheet interface. It was suggested that this FAC was driven by the Hall-effect, i.e. it closes the Hall current at a remote nearEarth X-line region (tailward of the spacecraft in this case) as will be described in more detail in Sect. 3.5. A statistical study also showed that such FAC, accompanied by Earthward and tailward ion flows, exists both at the Earthward and tailward side of the neutral line (Ueno et al., 2003). Magnetic field and particle observations have shown evidence of the FAC system associated with Hall-effect only in a region closer to the X-line (Nagai et al., 1998; Øieroset et al., 2001; Nagai et al., 2003; Alexeev et al., 2005; Henderson et al., 2006b). Hence these remote observations may provide evidence that the current closure of the Hall-current at the X-line region takes place on a more global scale, thus contributing to magnetosphere-ionosphere (M-I) coupling (Ueno et al., 2003; Nakamura et al., 2004b; Owen et al., 2005; Treumann et al., 2006).

Cluster observations by Nakamura et al. (2004b) were used to obtain the thickness of this transient FAC associated with energetic ion beams at the pre-midnight plasma sheet boundary layer, together with its temporal evolution, as summarized in Fig. 13. Sharp enhancements in $j_{x}$, with positive and negative peaks at the edge of the plasma sheet, were associated with energetic ion beams directed Earthward and 
with dawn-dusk moving low energy ions (upper four panels panels in Fig. 13). The FAC was associated with a clear reversal in $B_{y^{\prime}}$ at the two inner spacecraft, $\mathrm{C} 3$ and $\mathrm{C} 4$, and a single peak in $B_{y^{\prime}}$ at the two outer spacecraft, $\mathrm{C} 1$ and $\mathrm{C} 2$, which were located northward (larger value of $Z^{\prime}$ ) as shown in the lower panels, indicating the limited thickness of the FACs. Typical timescales of the FAC observations were 1$5 \mathrm{~min}$. The different profile in the magnetic field between the plasma sheet entry and exit suggests that this FAC is related to a transient process.

From the four-spacecraft analysis it was confirmed that the Earthward current layer had a thickness of $1600 \mathrm{~km}$, comparable to the relevant ion inertia length. Low energy proton flows indicated strong southward (northward) electric field exceeding $10 \mathrm{mV} / \mathrm{m}$, more than ten times larger than the nominal value, occurred at the outer (inner) part of this boundary region. The southward electric fields could be related to the Hall-effect (see Sect. 3.5) in the reconnection process, whereas the northward electric field could be related to the leading edge of the plasma flow jetting Earthward from the reconnection region.

A typical value of the Poynting flux for this event was estimated to be $0.1 \mathrm{erg} / \mathrm{cm}^{2} / \mathrm{s}$, directed Earthward (Nakamura et al., 2004b). This is comparable to the lower-altitude (4$7 R_{E}$ ) impulsive electric field observation by Polar (Wygant et al., 2000), if mapped along the field lines. The electric fields were interpreted as temporal variations due to Alfvén waves carrying Earthward Poynting flux with sufficient amplitude to explain the generation of the most intense aurora (Wygant et al., 2000). Wygant et al. (2002) showed that these Alfvén waves could further generate small-scale kinetic Alfvén waves, and suggested that in addition to the auroral particle energization processes at altitude between 0.5 and $2 R_{E}$, important heating and acceleration mechanisms can also operate these particle in the higher laltitude plasma sheet. Cluster, near perigee at a radial distance of about $4.5 R_{E}$, also observed such FAC related to perpendicular electric field spikes (Keiling et al., 2006). The FAC indicated standing and travelling Alfvén waves and appeared in association with energy-dispersed PSBL ion beams, and also had characteristics of beamlets (see Sect. 2.9).

Finally, the role of the FAC along the core field of a flux rope should be noted (see Sect. 2.2). Interestingly, although the FACs at the center of a flux-rope, which are often mainly dawn-dusk oriented, do not necessarily need connection to the Earth, it has been noted that there have been several reports of ionospheric signatures associated with a tailward moving flux rope/plasmoid (Lui et al., 1998; Ieda et al., 2001). Amm et al. (2006) also compared an Earthward moving flux rope observed by Cluster with ionospheric observations. This study identified a low precipitation region as the corresponding signature of a flux rope. Although the mechanism is as yet unknown, the magnetosphere-ionosphere coupling process may play an important role even in flux rope evolution.

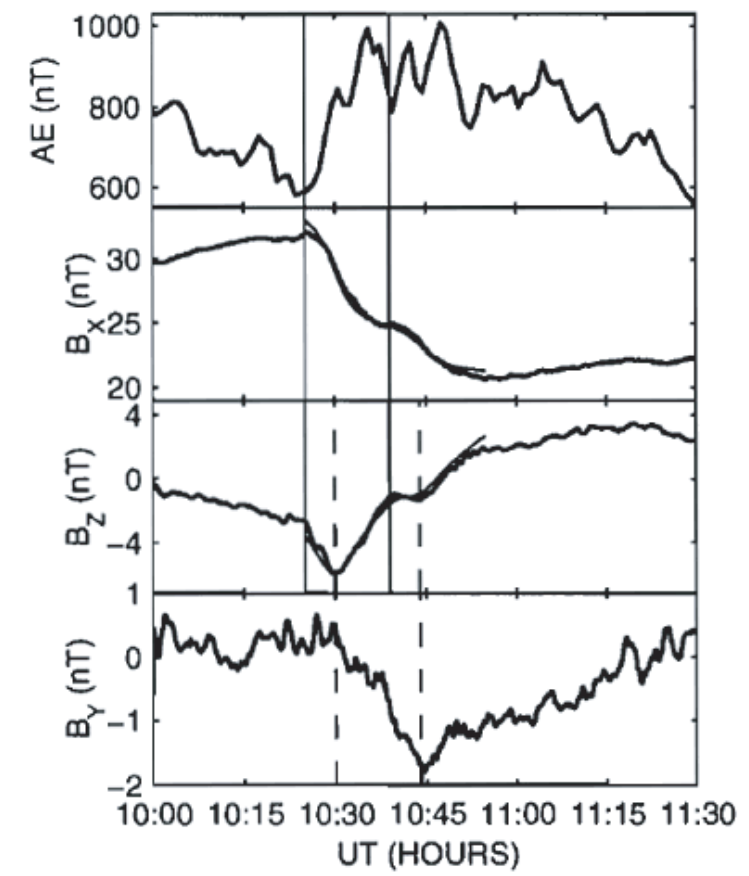

Fig. 14. Cross-tail current disruption effects measured in the lobe, onboard ISEE- 1 at $X=-20 R_{E}$. The AE index is shown at the top. The three bottom panels display the variations of the components of the lobe magnetic field. (From Jacquey et al., 1991).

\subsection{Current disruption}

The cross-tail Current Disruption (CD) corresponds to the local reduction of the tail current intensity. Evidence for this has been reported, first from near-Earth measurements, as one of the essential observed signatures during substorm expansion phases (e.g., Lui et al., 1978; Sauvaud and Winckler, 1980; Lopez and Lui, 1990; Ohtani et al., 1991). In the more distant tail lobe (10 to $30 R_{E}$ ), the typical magnetic signature is a negative excursion of $B_{z}$, followed by its rapid rise accompanied by the decrease in $B_{x}$, as illustrated in Fig. 14 and Fig. 15. The observed disturbances of $B_{x}$ and $B_{z}$ have been interpreted as due to the changes caused by a tailward expanding region of dusk-to-dawn currents which partially cancels the original dawn-to-dusk current and thereby disrupts the cross-tail current, as shown in Fig. 16. Using the multi-point measurements from the the ISEE, IMP and INTERBALL spacecraft, and by fitting the data to a simple disrupted current slab expanding tailward as well as Earthward, it was concluded that the CD started at 6-9 $R_{E}$ and propagated tens of $R_{E}$ down the tail with a velocity of the order of $150-350 \mathrm{~km} / \mathrm{s}$ during the substorm expansion phase (Jacquey et al., 1991, 1993, 1998; Ohtani et al., 1992). Direct comparisons of IMP-8 and ISEE-3 data during a major substorm revealed the link between CD and plasmoids seen in the far tail (Sauvaud et al., 1996). More recently, Cluster 


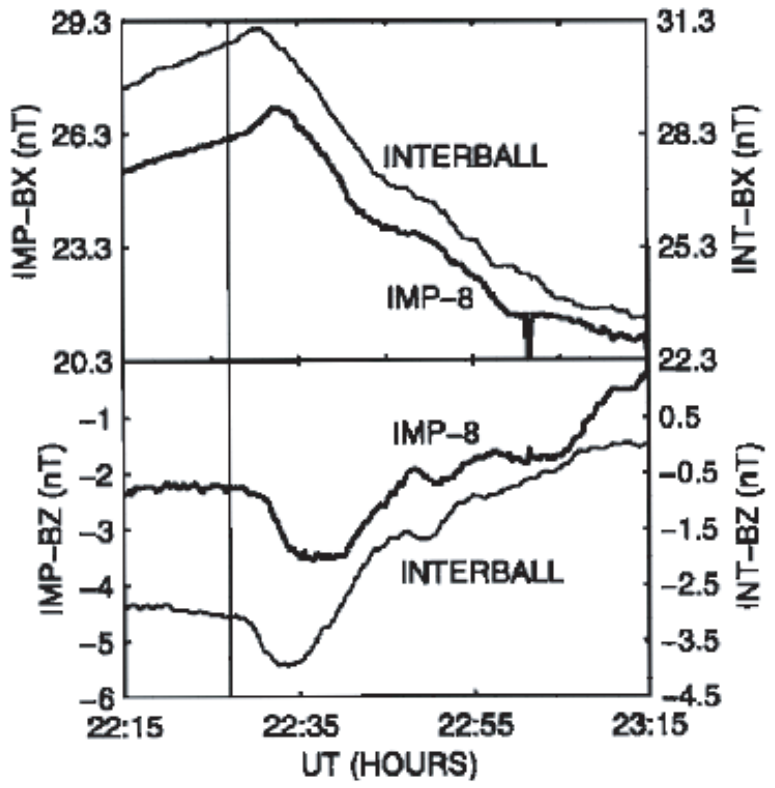

Fig. 15. Tailward propagation of a cross-tail current disruption as evidenced by simultaneous magnetic field measurements taken in the lobe onboard Interball-Tail and IMP-8, separated by about $10 R_{E}$ along the $X$ axis of the tail. (From Jacquey et al., 1998).

and Double Star data taken simultaneously in the tail have also been used to study both local and global expansion velocities and the plasma convection enhancement associated with these changes in the lobe topology (Sauvaud, 2008).

The CD signatures bear some superficial resemblance with the TCR bipolar variations of $B_{z}$ discussed in previous subsections (2.2-2.5) and displayed in Fig. 9. However, one of the essential features of a CD event is that $B_{x}$ should be reduced following the event, compared to the before level, indicating the "disruption" of the tail current. As shown in Fig. 9, the $B_{x}$ decrease is not a necessarily characteristic of SN TCR. Another major difference is that the CD negativeto-positive $B_{z}$ disturbance propagates tailward, while it propagates Earthward for a TCR. Thus multipoint measurements of the magnetic field signatures are extremely useful to distinguish these two types of events. Finally, the characteristic time scale of near-Earth CD and TCR are largely different: a TCR typically lasts for some tens of seconds (Fig. 9) while CD last for several minutes. Ohtani and Raeder (2004) successfully compared the CD signatures displayed in Fig. 15 with a global MHD modelling and suggested that the CD signature can be linked to magnetic reconnection. To verify this suggestion, plasma measurements are needed, particularly near the center of the current sheet. Hence multi-point measurements covering a lateral region relative to the current sheet and a combination of plasma measurements are essential for further investigations of these structures. (a)

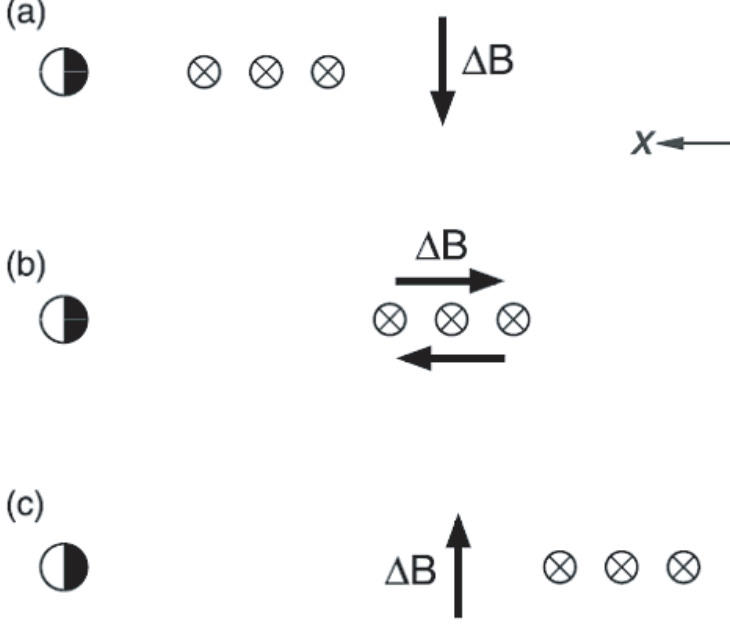

Fig. 16. Schematic representation of the relationship between $B_{x}$ and $B_{z}$ in terms of the tailward expansion of the current disruption region. (From Ohtani and Raeder, 2004).

\subsection{Current sheet flapping}

Large-amplitude (tens of nT) variations of $B_{x}$ (often with polarity changes) are observed frequently in the magnetotail. These are usually attributed to crossings of a flapping current sheet driven either by solar wind perturbations (Speiser and Ness, 1967) or by large scale-waves in the plasma sheet itself (Nakagawa and Nishida, 1989; Lui et al., 1978). Cluster magnetotail observations have revealed an abundance of relatively fast $(100 \mathrm{~s}-10 \mathrm{~min})$ crossings of the current sheet attributed to flapping events (Sergeev et al., 2004b; Runov et al., 2005). The current sheet thickness during these events was estimated to be several hundreds to few thousands of $\mathrm{km}$. Timing analysis of the magnetic field data from the four probes enable the estimation of the orientation of the current sheet and the direction of its motion (Harvey, 1998). The vector magnetic field derivatives (see e.g., Chanteur and Harvey, 1998) also provide important details of the electric current geometry.

Cluster observations shown in Fig. 17 illustrate the common features of such flapping perturbations. The $X$ component of the magnetic field exhibits repeated largeamplitude variations with sign changes, indicating multiple crossings of the neutral sheet. The similarity of the $B_{x}$ curves at different spacecraft, separated by $\sim 2000 \mathrm{~km}$, shows that the magnetic field variations are due to a spatial structure passing the Cluster constellation. The trace at the most duskward probe ( $\mathrm{C} 2$, red) is delayed with respect to the others, indicating a structure approaching Cluster from the dawnside. Timing analysis of the magnetic field variations shows that the current sheet is strongly tilted with respect to its nominal position, as illustrated in the top panel in Fig. 17, and shows duskward propagating kinks on the 


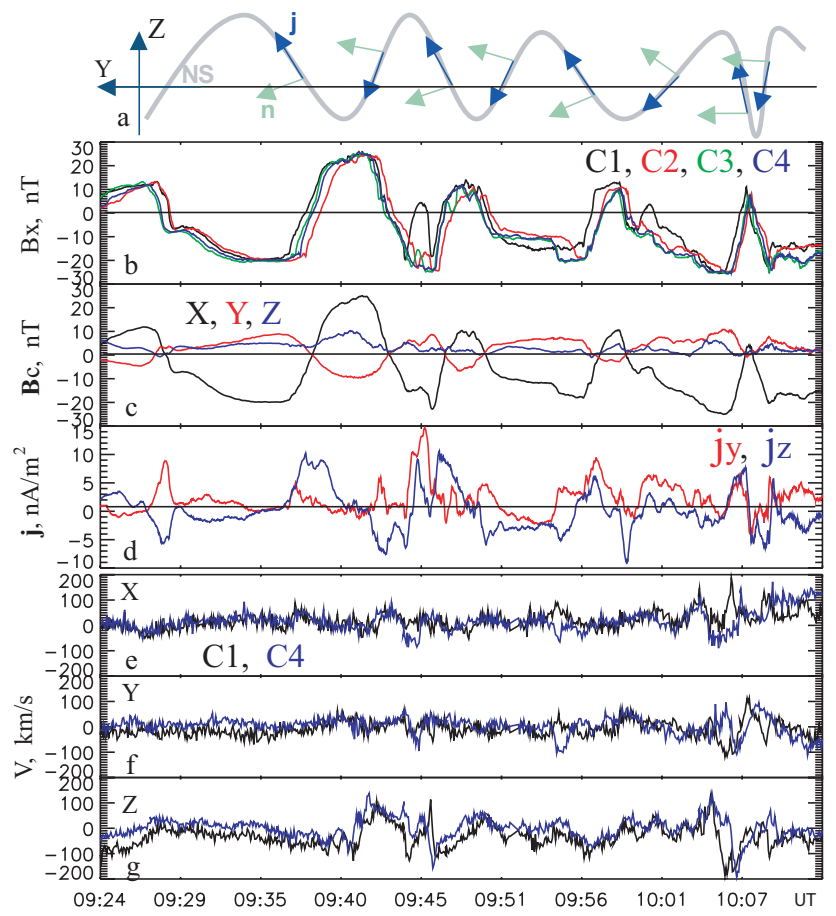

Fig. 17. Cluster observations of the current sheet flapping on 20 October 2001. The $X$-component of the magnetic field at all four spacecraft (b); the $X, Y$, and $Z$ components of the magnetic field at the Cluster barycenter (c); the $Y$ and $Z$ components of the current density $\mathbf{j}=\mu_{\mathbf{0}}^{-\mathbf{1}} \nabla \times \mathbf{B}$ (d); the $X, Y$, and $Z$ components of the ion bulk velocity at $\mathrm{C} 1$ and $\mathrm{C} 4(\mathbf{e}, \mathbf{f}$, and $\mathbf{g})$; current sheet profile with projections of current density and current sheet normal vectors onto the $(Y, Z)$ plane (a). The GSM coordinate system is used. (From Sergeev et al., 2004b).

current sheet surface. The normal velocity of these kinks varies between $40-80 \mathrm{~km} / \mathrm{s}$. In agreement with the direction of the normals, the electric current vector $\mathbf{j}=\mu_{0}^{-1} \nabla \times \mathbf{B}$ rotates in the $(Y-Z)$ plane: $j_{z}\left(\left|j_{z}\right| \geq j_{y}\right)$ is positive during $\partial B_{x} / \partial t<0$ and vice versa. The plasma sheet was quiet, with $\left|V_{x}\right|$ and $\left|V_{y}\right|<200 \mathrm{~km} / \mathrm{s}$. The variations of $V_{z}$ are negative during $\partial B_{x} / \partial t<0$ (south - north crossings) and positive during $\partial B_{x} / \partial t<0$ (north-south crossings), which are consistent with a vertical motion of the flux tubes. Integration of $V_{z}$ over time gives an estimate of the kink amplitude of $1-2 R_{E}$.

The above example demonstrates duskward propagating current sheet kinks when Cluster was situated near the dusk flank. Zhang et al. (2002) have reported dawnward propagating kinks observed by Cluster in the morning sector of the magnetotail. Isolated kinks, or pairs of them, have also been observed frequently (Petrukovich et al., 2003; Runov et al., 2005).

Statistical analysis of flapping events observed by Cluster shows that the flapping transients propagate typically toward the flanks from the near-midnight sector (Sergeev et al., 2004b; Runov et al., 2005). The distribution of timing nor-
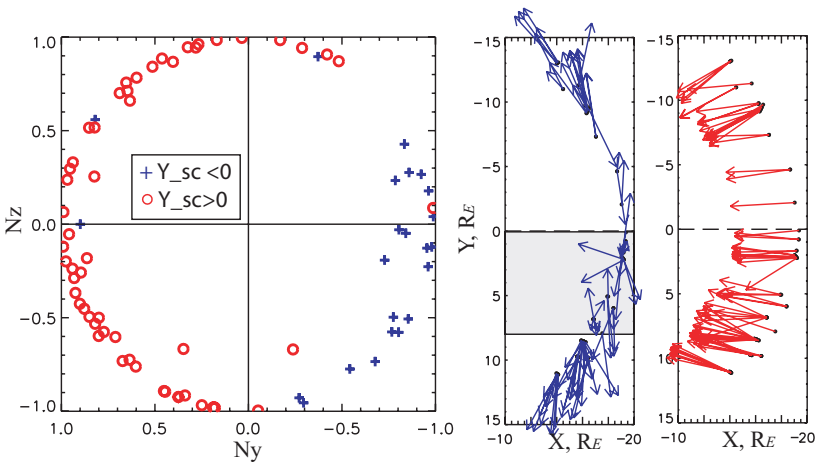

Fig. 18. Results of statistical analysis of 78 flapping current sheet crossings during July-October, 2001. Distributions of current sheet normals obtained by timing in $\left(N_{y}, N_{z}\right)$ plane (left panel, samples from the dawn sector are marked by crosses and that from the dusk sector are marked by circles). Projections of the current sheet normal velocity vectors (middle panel) and maximum variance magnetic field (right panel) onto the $(X, Y)_{\mathrm{GSM}}$ plane. (From Runov et al., 2005).

mals in the $N_{y}-N_{z}$ plane (Fig. 18, left panel) shows the current sheet normals are tilted predominantly in the $-Y$ direction in the dawn sector $\left(Y_{s / c}<0\right.$, circles $)$ and in the $+Y$ direction in the dusk sector $\left(Y_{s / c}>0\right.$, pluses). The points are distributed near the unit circle, thus $N_{x} \ll \sqrt{N_{y}^{2}+N_{z}^{2}}$. Projections of the current sheet normal velocities during crossings onto the $(X-Y)_{\mathrm{GSM}}$ plane (mid panel) shaw predominantly flankward directions, with significant $V_{n x}$ component near the flanks, except for the sector between 0 and $8 R_{E}$ (shaded box). A comparison with projections of the maximum variance magnetic field component onto the $(X-Y)_{\mathrm{GSM}}$ plane (right panel) shows that the flapping transients propagate typically perpendicular to the flaring magnetic field, i.e., in the azimuthal or y direction. The current sheet normal velocity was most frequently in the range $30-70 \mathrm{~km} / \mathrm{s}$. Magnetospheric conditions during flapping events vary from quiet (no plasma flows) to very active intervals with intermittent plasma flows.

The considerable $Y-Z$ tilt of the current sheet during fast current sheet crossings was also found in a retrospective analysis of Geotail observations (Sergeev et al., 2006). The occurrence of fast crossings increases with downtail distance and has a peak near the tail center (with some duskward shift). This resembles the occurrence statistics of BBFs, although there is no one-to-one correspondence between these two phenomena locally. The crossing durations are spread around $1 \mathrm{~min}$ but decrease for high-speed flows. Flapping motions appear preferentially during the substorm expansion phase, although a considerable number of events without any electrojet and auroral activity were also observed.

In a number of observations, simultaneous flapping events were detected by spacecraft aligned in local time and radially separated by $10 R_{E}$ (Interball - Geotail pair, Petrukovich 

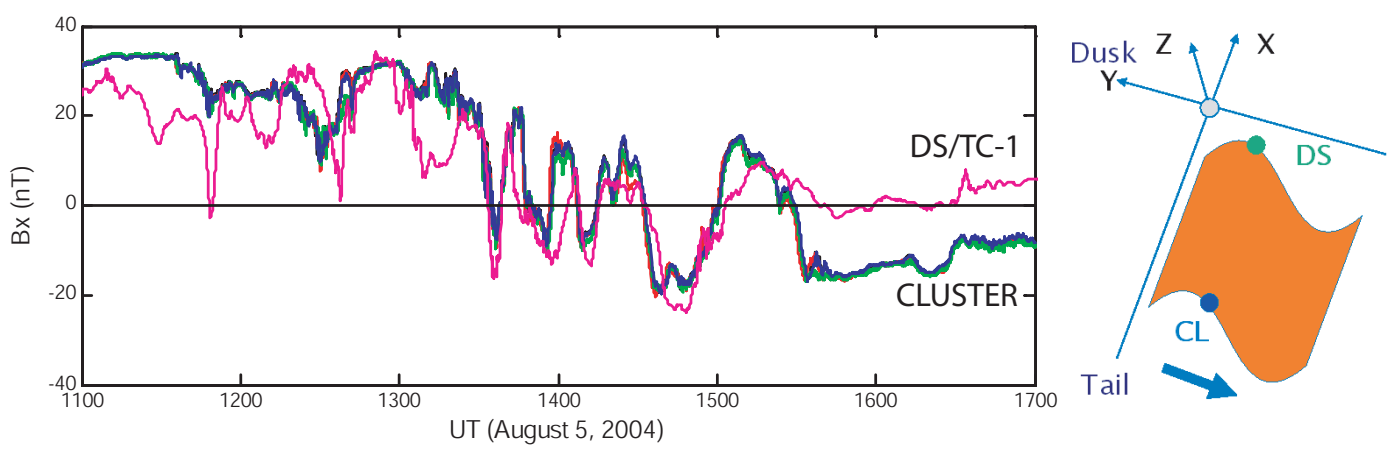

Fig. 19. Simultaneous current sheet crossings by Cluster and DS/TC-1 spacecraft, radially separated by $\sim 5 R_{E}$. (Adopted from Zhang et al., 2005).

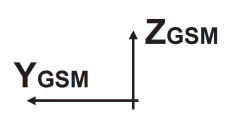
Nominal horizontal
configuration

Slip-type deformation: Flux-tubes shift, magnetic direction is constant, thickness decreases.
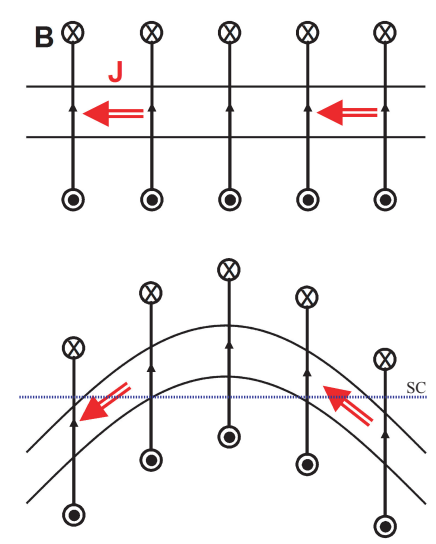

Bend-type deformation: Flux-tubes rotate, magnetic direction follows the normal, thickness is constant.

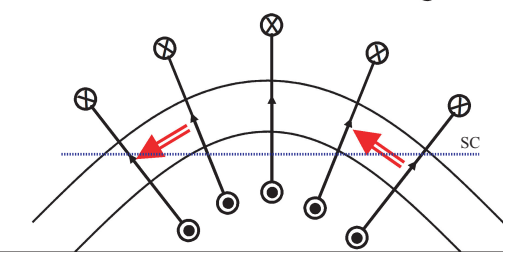

Fig. 20. Scenarios for cross-tail current sheet deformations. The view in the $Y Z$ GSM plane is shown, assuming nominal configuration with $B_{y}=0$. The blue dashed lines show spacecraft passes. (From Petrukovich et al., 2006).

et al., 2003) and $6 R_{E}$ (Cluster - Double Star-1 pair, Zhang et al., 2005, Fig. 19). The observed magnetic field polarity change is, therefore, due to radially elongated corrugation of the current sheet, propagating flankward with respect to the spacecraft, rather than due to an localized dynamic "hump" (Lui et al., 1978; Lui, 1984).

Such complex shapes of the plasma sheet are in deep contrast to the commonly assumed near-planar geometry. Large tilts of the magnetotail current sheet were previously considered only in the context of a macro-scale twist. However, most observed events are meso-scale, i.e., a few $R_{E}$ in the cross-tail direction. Crossings with alternating tilts are often observed within minutes. Fast crossings of rather thin sheets (with thicknesses of a few thousands of $\mathrm{km}$ ) were often ob- served even in situations where an expanded plasma sheet is expected (e.g., during northward IMF and near-flank crossings).

Considering the current sheet as a three-dimensional object formed by curved magnetic flux tubes, two types of mesoscale deformations, as illustrated in Fig. 20, may take place. During a bend-type deformation the flux tubes rotate, following the change in the sheet normal direction. Alternatively, during a slip-type deformation, the flux tubes shift vertically relative to their neighbors, but the magnetic field orientation remains the same, irrespective of the value of the tilt. Thus, the changes in the guide (current-aligned, $B_{m}$ ) and normal $\left(B_{n}\right)$ components of the magnetic field during a crossing provide the details of the nature of sheet deformation.

Analyses of a series of crossings, with the guide and normal field components large enough to detect changes in the field direction, support the slippage scenario. Particularly, Cluster observations of several strongly-tilted current sheets with a large $B_{z}$ but a vanishing $B_{n}$ were interpreted as crossings of an azimuthally-flapping, quasi-stationary boundary between adjacent magnetic field flux tubes, indented vertically relative to their neighbors (Petrukovich et al., 2003). Furthermore, studies of several wave-like current sheet flappings show that, in agreement with the slippage model, the magnetic field inside the sheet rotates during subsequent crossings by only $10-20^{\circ}$, while the difference between the normals of pairs of neighboring crossings varies in the range of $50-150^{\circ}$ (Petrukovich et al., 2006).

It should also be noted that, due to the slip-type deformation, the current sheet thickness (along the tilted normal) decreases and the current density increases with the cosine of the sheet tilt angle (Fig. 20). Coherent slipping motion of all flux tubes in the same local time sector may produce observed radial elongation of sheet kinks (Fig. 19). As a result, during flapping events of this type, the spacecraft cross a thin, intensified and tilted current layer - a dynamic modification of the inner plasma sheet (see also Fig. 9 in Petrukovich et al., 2006). Statistical studies show that for more tilted sheets the guide component tends to be larger 
and the normal component smaller (Runov et al., 2006). As shown in Fig. 21, the $B_{m}$ values show clear dependence on the sign and value of the tilt angle. In agreement with the slippage scenario again, a large, stable $B_{z}$ mainly contributes to a large guide component at tilted sheets.

In conclusion, interpretations of fast (100 s-10 min) current sheet flappings may differ depending on observed magnetic field geometry, local plasma conditions, as well as the magnetospheric conditions. In many cases this phenomenon is consistent with the crossing of a thin, dynamic, embedded current layer, rather than a motion of the magnetotail plasma sheet as a whole. Quiet-time, strongly-tilted sheets form a significant subset of flapping events, and are consistent with a model of slip-type displacement of magnetic flux tubes, moving azimuthally. On the other hand, events with small $B_{z}$ and $B_{y}(1-2 \mathrm{nT})$ and/or small tilt are more difficult to analyze but some of them are not consistent with the slippage scenario. Alternative interpretations include crossings of bent thin current sheets, plasma flow channel boundaries (in cases of large tilts), or vertical motion of a thin horizontal plasma sheet. Investigations of such cases are necessary for a better understanding of current sheet flapping.

\subsection{High frequency oscillations observed by Cluster}

Various types of fluctuations may propagate in the plasma sheet, with periods ranging from seconds to minutes. They are often organized as wave packets or bursts and, in that sense, may be considered as transient phenomena in the plasma sheet. It is also widely recognized that they are closely related to magnetotail activity and, in particular, to BBFs. Examples of waves and fluctuations observed in the plasma sheet can be found in Bauer et al. (1995); Ohtani et al. (1996); Kepko and Kivelson (1999); Volwerk et al. (2004); see also the review by Lui (2002) concerning the relationship between wave activity and other transient phenomena.

Being an active system, the magnetotail current sheet almost permanently shaken by fluctuations with periods ranging from seconds to minutes. An example of particularly well-defined high frequency undulations is shown in Fig. 22. This event was observed by Cluster on 22 August 2001, around 09:40 UT, at $X \simeq-18.7 R_{E}$ in the morning side of the plasma sheet $\left(Y_{\mathrm{GSE}} \simeq-3.6 R_{E}\right)$. On panels 5 and 6 , the sound speed, the typical thickness of the sheet (deduced from a fit of the magnetic field with a Harris sheet), and the characteristic MHD scale are displayed.

Ground based data show that the magnetosphere is quiet from 08:00 to 09:30 UT and active after 09:30 UT, with perhaps a pseudo breakup at about 09:26 UT. A major substorm develops prior to 09:40 UT with onset at $\sim 09: 37$ UT, the activity then persisting during the whole crossing of the sheet. Quiet and active states of the sheet clearly correspond to the observation of different types of fluctuations.

During the quiet period, the fluctuations have typical temporal scales of a few minutes. When the magnetospheric ac-

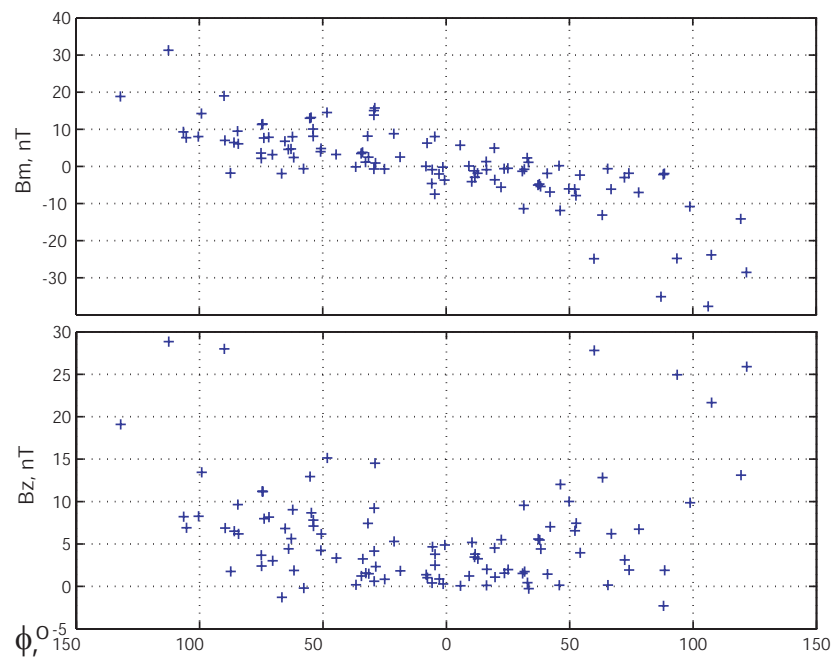

Fig. 21. Magnetic field components $B_{m}, B_{z}$ relative to the current sheet normal tilt in the $Y Z$ plane (zero corresponds to normal parallel to $Z_{\mathrm{GSM}}$ ). Events from the 2001 (Runov et al., 2006) and 2004 datasets are shown.

tivity develops (after 09:40 UT), very large amplitude waves with periods smaller than one minute are detected. Louarn et al. (2004) have shown that the long period fluctuations, with periods significantly larger than one minute, are unlikely to be MHD eigenmodes of the sheet since the wavelength of such modes would then largely exceed the ClusterEarth distance. These perturbations could be quasi-static adjustments of the sheet to modifications in the solar wind conditions. Conversely, the short period oscillations, observed during the peak of activity, are compatible with MHD eigenmodes with typical wavelength of $1-2 R_{E}$. During the corresponding period, the characteristic half-width $a$ of the sheet is typically $a=0.2 R_{E} \simeq 1280 \mathrm{~km}$. With a typical sound speed of $v_{s} \simeq 800 \mathrm{~km} / \mathrm{s}$, the MHD time scale is thus $\tau=a / v_{s}=1.6 \mathrm{~s}$, which corresponds to a frequency of $0.625 \mathrm{~Hz}$.

The details of these magnetic oscillations are presented in panels 7 to 9 of Fig. 22. Louarn et al. (2004) studied the time evolution of the sheet by fitting the 4 magnetic measurements with a Harris sheet model which leads to determine the position of the neutral sheet with respect to SC1 (z) and the half thickness of the sheet (1), as shown in panel 8. The "serpentine" oscillations of the sheet are displayed in panel 9 where the boundaries of the colored area are, respectively, $z+1$ and $\mathrm{z}-1$. This plot thus corresponds to the temporal evolution of the sheet at the position of Cluster. Fourier analysis of the position and of thickness of the sheet yields the spectrum shown in the bottom panels. The neutral sheet undulates between $\mathrm{SC} 1$ and SC4 with a typical period $20-25 \mathrm{~s}$ period (spectral peak at $0.04 \mathrm{~Hz}$ ). The sheet thickness fluctuates at the same frequency but also presents a peak at $0.13-0.14 \mathrm{~Hz}$. In terms of MHD eigenmodes, these results suggest that the oscillations are composed of two sausage-like oscillations, at 0.04 and $0.13 \mathrm{~Hz}$, and one kink-like oscillation, at $0.04 \mathrm{~Hz}$. 
Magnetic field (X comp.) - day 22/08/2001
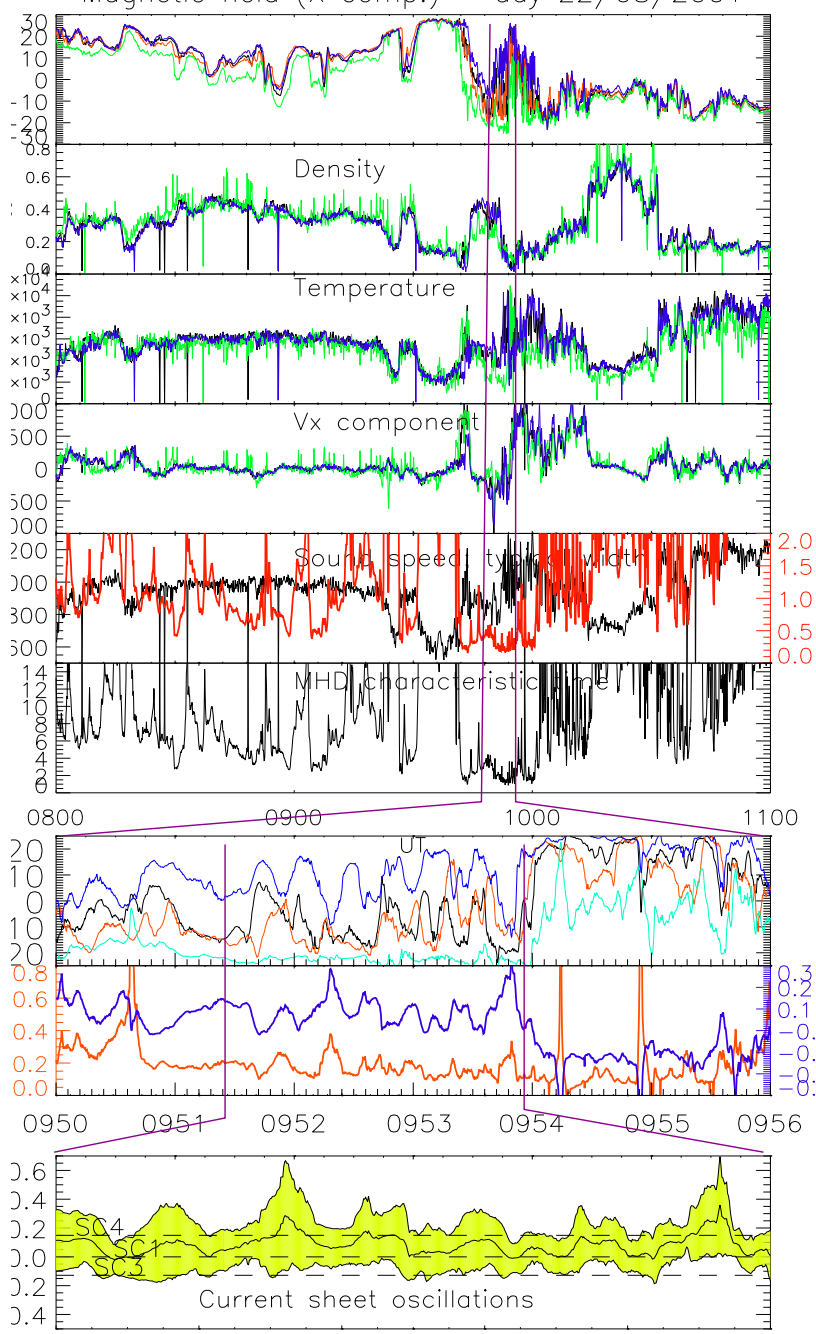

$0.4=$
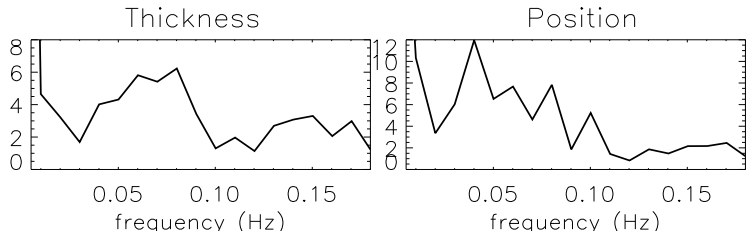

Fig. 22. Overview of the 22 August 2001 event. Panels 1 to 6: overview of the crossing. Panel (1): X component of the magnetic field (4 SC determinations), Panel (2): ion density (SC1, SC3 and SC4) Panel (3): ion temperature, Panel (4): ion velocity, Panel (5): sound speed (in black) and thickness of the sheet (in red), Panel (6): MHD characteristic time. Panels 7 to 9: Zoom on the oscillations. Panel (7): X component of the magnetic field, Panel (8): position (z, in blue) and half thickness (1, in red) of the sheet deduced from a fit with a Harris sheet, Panel (9): temporal evolution of the sheet. The vertical axis corresponds to $\mathrm{Z}$ (gsm). The colored area represents the sheet, localized between $\mathrm{z}-1$ (lower limit) and $\mathrm{z}+1$ (upper limit). The central line is z. Panel (10): spectral analysis of the position and thickness. (From Louarn et al., 2004).

\subsection{VDIS, TDIS, and beamlets}

Energization processes occurring in the magnetotail current sheet leave their imprints in the PSBL, producing complicated spatio-temporal plasma structures. These are clearly manifested in the variations of particle flux and spectra which are observed in the auroral PSBL (at the low-altitude end of the plasma sheet horns). In particular, observations in this region show a wealth of dispersed ion structures (in which the ions having different energies were observed at different latitudes or at different times), and are roughly split into two classes: Velocity Dispersed Ion Structures (VDIS) and Time Dispersed Ion Structures (TDIS). The dispersion is more easily formed for the $\mathrm{keV}$ ions whose characteristic dispersion time (minutes) is large enough to be observed during PSBL crossings. It is basically formed due to three main effects, as shown schematically in Fig. 23. First, the pure time-offlight (TOF) effect which spreads ions having different energies along the magnetic field line. Second, the velocity filter (VF) effect due to magnetotail convection, which produces the equatorward $\mathbf{E} \times \mathbf{B}$ drift of accelerated ions while they are moving from the source along the magnetic field lines. Third, the "place of birth" (BP) effect related to the ion acceleration mechanism whose efficiency may depend on the local conditions in the current sheet, such that the energy gained by the ions may vary with the location of their acceleration site. The VF effect produces the spatial structuring (layering) of ions according to their energy value, with the highest-energy ions near the PSBL-lobe interface and lower energy ions deeper in the PSBL. Such spatial dispersion is commonly associated with VDIS (Fig. 23a). The transient acceleration and pure TOF effect (more energetic particles are seen first) was initially considered as origin of TDIS structures (Fig. 23b), but in the presence of convection it also inevitably produces a latitudinal energy dispersion. Real events, in which temporal variations of acceleration are combined with variable boundary and plasma motions, always exhibit a mixture of temporal and spatial effects.

The temporal origin of Temporal Dispersed Ion Structures (TDIS) (Sauvaud et al., 1999) is easier to identify when observing at low altitudes in the strong magnetic field region.

Examples of TDIS-type sub-structures obtained during poleward crossing of Cluster perigee pass and equatoward crossing of Interball Auroral are shown in Fig. 24. The transient origin of the multiple, minute-scale, injections shown in this figure is evidenced by the fact that their sense of dispersion (energy decreasing with time) does not depend on the direction of the crossing through the auroral zone (in poleward or equatorward directions, compare Fig. 24a and b). Their temporal origin was also confirmed by the one-to-one correspondence of multiple dispersed beams with concurrent auroral activations in the conjugate ionosphere (Sergeev et al., 2000b). Its localized feature was emphasized by multipoint Cluster observation (Keiling et al., 2004). According to a summary by Sauvaud and Kovrazhkin (2004), TDIS are 

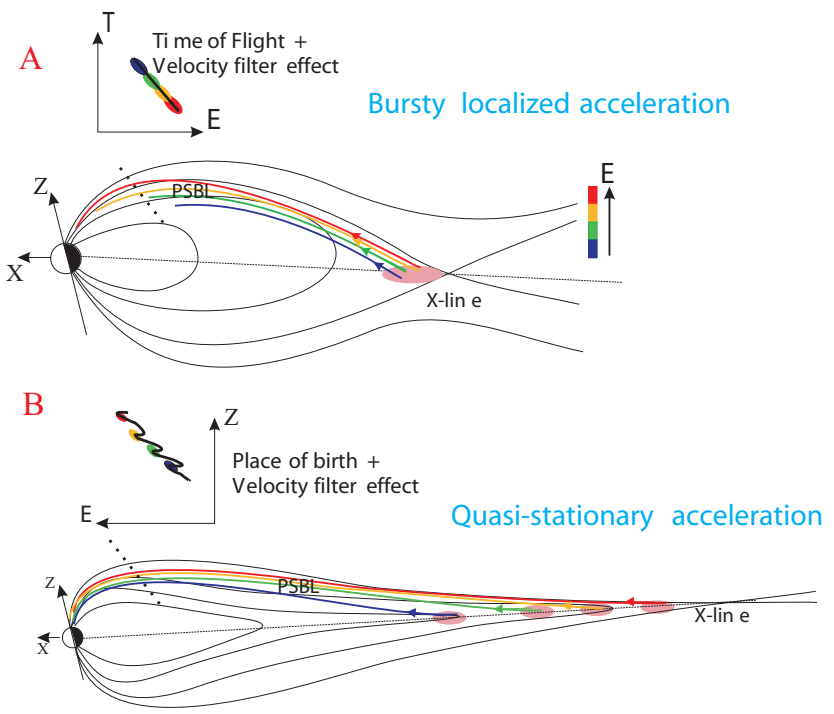

Fig. 23. Two schemes for the formation of ion dispersion in the PSBL. (A) Transient ion acceleration at a localized source located near an X-line (indicated by the pink oval) results in ions with wide distributions of parallel energies $(E)$ being ejected into the PSBL. In this case the dispersion is mostly formed by the Time-of-Flight and Velocity Filter effects. (B) Quasi-stationary ion acceleration in the wide vicinity of an X-line at multiple localized resonant sources; ions ejected from each particular resonance will have a relatively narrow distribution of their parallel energies. In this case the dispersion in the PSBL will be formed due to Place of Birth and Velocity Filter effects and multiple localized beams (beamlets) may be observed.

common in the poleward part of the oval (especially during substorms), have durations of 1-3 min and apparent recurrence period of $\sim 3 \mathrm{~min}$, consistent with the BBF temporal scales (Sect. 2.1, simultaneous conjugate observations of both phenomena were studied by Sergeev et al., 2000a). When neglecting the contribution of spatial effects, the TOF analyses gives injection distances of $8-40 R_{E}$ if observed at $R \sim 2-3 R_{E}$ (Sauvaud and Kovrazhkin, 2004), or 40-100 $R_{E}$ if observed at $R \sim 10-15 R_{E}$ in the tail (Kazama and Mukai, 2005). In the latter case, dispersed Earthward-directed ion beams with wide pitch-angle distributions were frequently associated with local Earthward bulk flows and turbulent magnetic dipolarizations. A considerable fraction of these Earthward beams coming from the magnetotail (40\% of the total) were accompanied by highly-collimated, tailwardmoving, cold dispersed ions, probably extracted from the ionosphere.

In many cases of sporadic injections, the contribution of spatial effects to the observed dispersion can be comparable or dominate over the pure TOF effect. A clear example of this was the very energetic, intense ion beam observed near the separatrix footpoint in the equatorial part of the oval during a strong near-Earth reconnection event observed by Cluster (Sergeev et al., 2007). The unusually steep dispersion slope
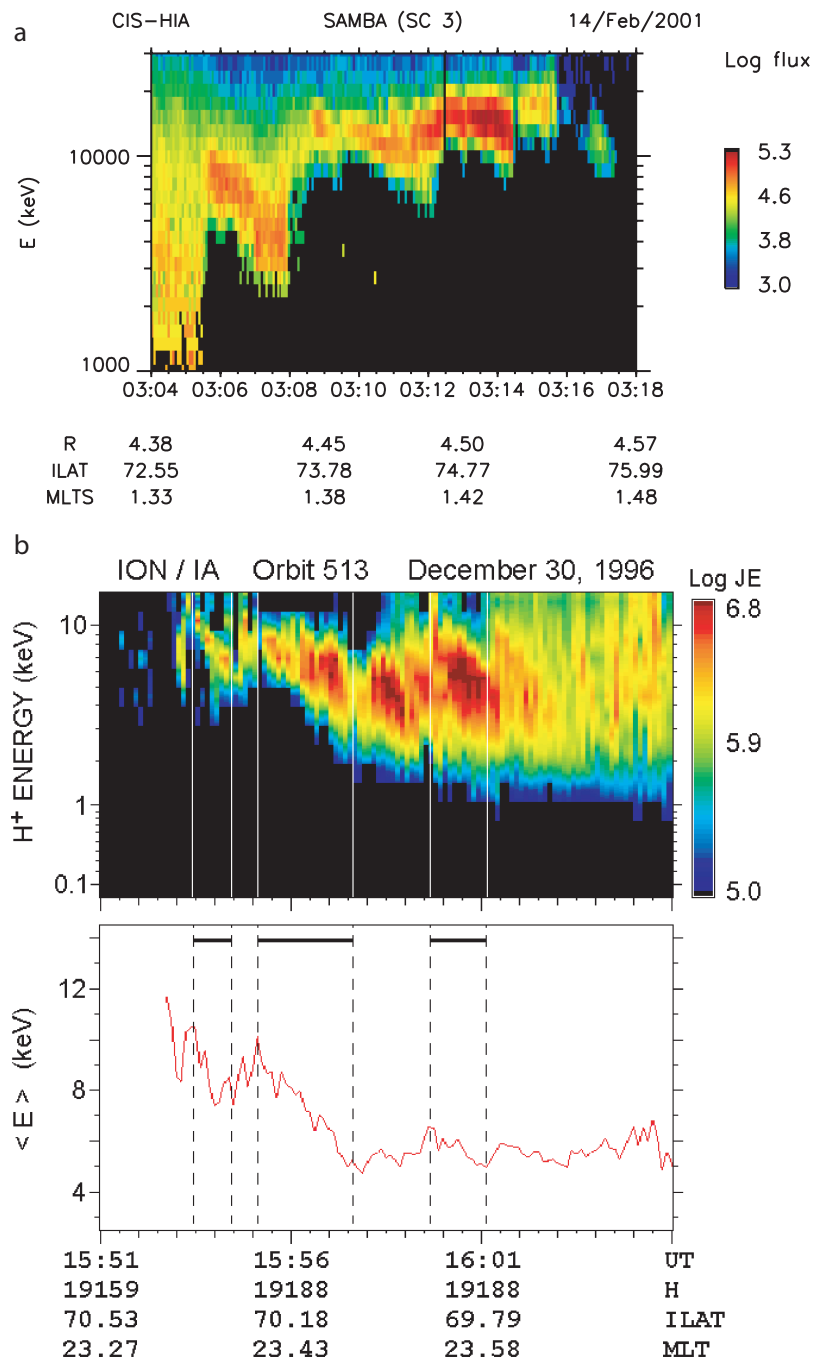

Fig. 24. (a) Example of a high energy VDIS structure measured on board Cluster during a poleward crossing of the auroral zone-polar cap boundary. VDIS consists of substructures where high-energy protons arrive first and the time-of-flight effect of protons generally dominates over the velocity filter effect. (b) Another example of a VDIS structure shown in proton energy-time spectrogram (top panel) and average energy (bottom panel) on board Interball Auroral during an equatorward crossing. The ion average energy decreases in time, while it contains substructures in which higher-energy protons arrive before the low-energy ones as expected from a timeofflight dispersion (From Sauvaud and Kovrazhkin, 2004).

(implying only $\sim 2 R_{E}$ source distance in the case of pure TOF interpretation) was basically formed by the spatial motion of the source footpoint (due to the poleward expansion of separatrix footpoint) with respect to the observing DMSP spacecraft.

The most well-known examples of VDIS, (Zelenyi et al., 1990) are those located near the poleward oval boundary (outer boundary of the PS). Such structures manifest 

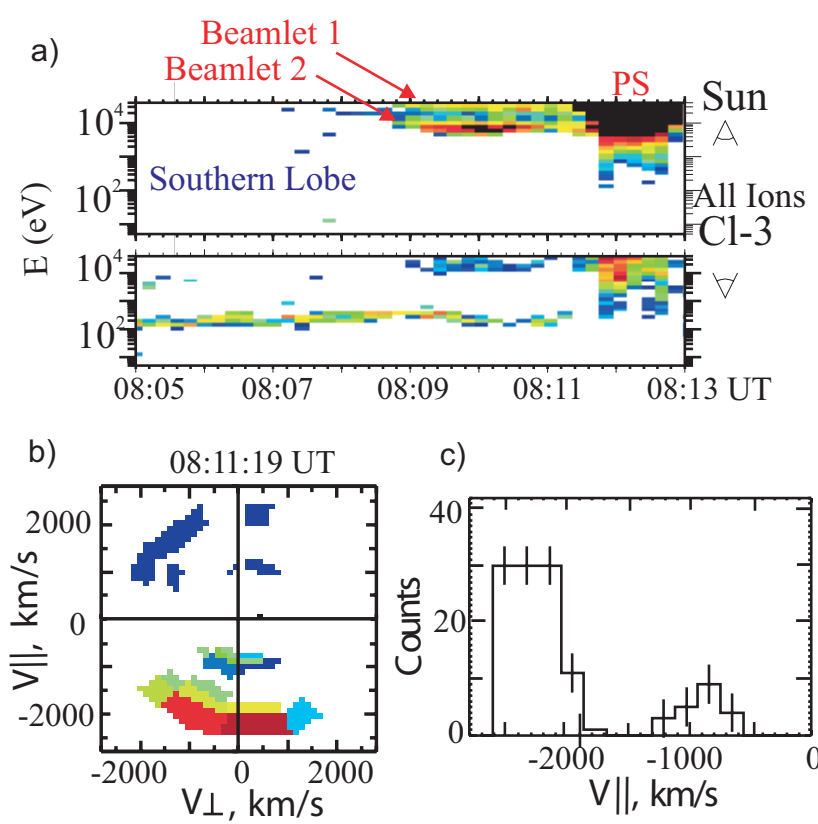

c)

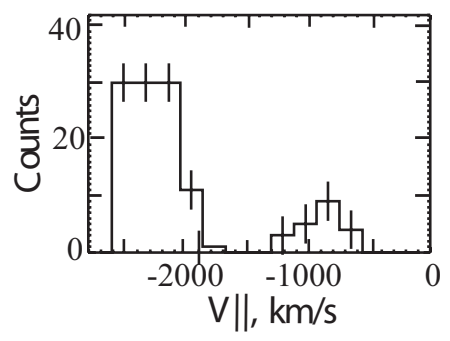

Fig. 25. Cluster observation of "double-peaked" ion distributions in the PSBL on March 9, 2001. E-T spectrograms of Earthward and tailward moving ions obtained by Cluster/HIA (a). 2-D (cut in $V_{\|}-$ $V_{\perp}$ plane, b) and 1-D (cut along magnetic field, c) $12 \mathrm{~s}$ accumulated ion distribution. (From Zelenyi et al., 2006).

increases in the low-energy cutoff with increasing latitude (Fig. 24) and have $\sim 1-2^{\circ}$ width in invariant latitude. They are most clearly visible during quiet and substorm recovery conditions (Sauvaud and Kovrazhkin, 2004). In the tail, the corresponding spatial dispersion is frequently observed near the plasma sheet-lobe interface for both ions and electrons (e.g., Onsager et al., 1990; Elphic et al., 1995), and recent Cluster observations confirm the related dispersion in lobe polar rain electron population (Alexeev et al., 2006). Other kinds of spatial dispersion structures in the main body of plasma sheet are also known from low-altitude observations (see e.g., Hirahara et al., 1996).

The properties of PSBL dispersed structures are also influenced by the "place of birth" effect. Two basic scenarios of ion acceleration were put forward to explain the observed features. Onsager et al. (1990) assumed a localized source (distant reconnection region) accelerating ions over a wide energy range. In this case, the dispersion of PSBL ions streaming Earthward will be solely due to the velocity filter effect. Another scenario, proposed by AshourAbdalla et al. (1993), suggested ion acceleration in multiple, spatially-localized sources distributed over a wide area Earthward of the distant X-line. Such a scenario is appropriate for a relatively stable and stretched magnetotail configuration with a large region of weak transverse magnetic field component in which effective non-adiabatic ion acceleration could occur.
Each source (termed resonance) accelerates ions in a relatively narrow energy range, controlled by the local conditions in the source (i.e. by the values of local magnetic and electric fields in the center of the current sheet). Since the magnetic field within the current sheet has a spatial profile, ions accelerated at different resonances have different energies. Taking into account these spatial profiles, a new model has been developed in which the narrow spatial localization and multiplicity of beams, called "beamlets", can be explained. Such non-adiabatic resonance acceleration processes in the current sheets are described below, and in Sect. 3.10 in more detail.

Recent Cluster observations in the magnetotail and the auroral extensions of the PSBL have provided evidence of spatial structuring and the presence of beamlets, which are often seen in the PSBL near Cluster apogee (Zelenyi et al., 2006). One such case is presented in Fig. 25 showing Cluster observations in the southern lobe (at $[-18,0,-4] R_{E}$ ) made on 1 September 2003. At 08:09 UT, the closely-spaced Cluster spacecraft entered the PSBL and observed two field-aligned ion beams streaming Earthward and clearly separated in energy (Fig. 25a). These two energetic ion populations, one with an energy of about $5 \mathrm{keV}$ and the other with an energy close to the instrument threshold $(\sim 30 \mathrm{keV})$ were simultaneously observed for $\sim 2.5 \mathrm{~min}$ by three closely-spaced Cluster satellites (due to their similarity only observations at one spacecraft are presented in Fig. 25). The 2-D cut of the 3-D ion velocity distribution functions in the plane containing both the magnetic field and the convection velocity (Fig. 25b) illustrate the beam nature of two ion populations streaming along the magnetic field. A cut of the distributions along the magnetic field direction (Fig. 25c) shows two clearly separated peaks of field-aligned ions at the velocities of $\sim 2400 \mathrm{~km} / \mathrm{s}$ and $\sim 1000 \mathrm{~km} / \mathrm{s}$. A similar double structure is also observed later in the reflected (tailward) beams. This feature exists in the ion distribution for at least $3 \mathrm{~min}$, suggesting a spatial origin. Due to the convection transporting the plasma across $B$ (velocity filter effect), which is always the case in the magnetotail, these two beams can not be accelerated at one and the same source and their simultaneous observation in the PSBL provides a strong argument in favor of simultaneous operation of at least two resonant sources in the tail current sheet.

\subsection{Energetic particles}

Another type of transient phenomenon, in the higher energy particles, has been observed in the Earth's magnetotail. In early satellite observations, Sarris et al. (1976) and Hones et al. (1976) reported anisotropic energetic particle bursts. Similar energetic electron bursts with energies $0.3 \sim 1.0 \mathrm{MeV}$ were also detected during southward turnings of the $B_{z}$ at $X=-20--30 R_{E}$ in the magnetotail (Terasawa and Nishida, 1976). Energetic particle events at $X \sim-30 R_{E}$ are also discussed by Baker and Stone $(1976,1977)$ based on the survey of electrons of $\varepsilon \geq 200 \mathrm{keV}$. They reported that the $\varepsilon \geq 1 \mathrm{MeV}$ 
electron enhancement are usually associated with neutral sheet crossings. This would suggest that the energetic particles are associated with magnetic reconnection. More recent, similar observations have been discussed (e.g., Möbius et al., 1983). Øieroset et al. (2001) reported comprehensive observations of energetic particles up to $\sim 300 \mathrm{keV}$ in the vicinity of an $X$-type region, and the fluxes of energetic electrons around the diffusion region.

Magnetic reconnection is understood as an engine of energetic particle production, although it is still an open question where the energetic particles are generated in the reconnection region. Figure 26 shows the profile of a plasma sheet crossing observed by Geotail, located at $X=-24 \mathrm{R}_{\mathrm{E}}$ in magnetotail. Shown in this figure, from the top, are the electron energy-time diagram, the magnetic field $B_{x}$, the plasmas bulk flow $V_{x}$, ion temperature $T_{i}$, and the integrated flux of electrons with energy $>35 \mathrm{keV}$. The satellite crosses close to an inferred X-type neutral sheet, indicated by the reversal in $V_{x}$ and associated weak $B_{x}$, around 17:50 UT. The key point is the integrated energetic particle flux in the bottom panel, showing that the flux of the energetic electrons is enhanced outside of the X-type neutral line.

Recently Imada et al. (2005) investigated the relationship between thermal plasma heating and energetic particle acceleration around the reconnection region in the tail, based on a superposed epoch analysis of Geotail observations. Figure 27 shows the profiles of the energetic electron flux $(>38 \mathrm{keV})$ and the electron temperature around the reconnection region. The energetic particles and high temperature plasma are observed in and around the reconnection region, but the enhanced energetic flux and higher temperature regions are situated around the reconnection outflow region downstream away from the center of the X-type neutral line. This analysis suggests that energetic particles are generated not only in the X-type region but also in a wide area surrounding the diffusion region. This behavior has been confirmed using multi-satellite Cluster observation by Imada et al. (2007). Specifically, they concluded that the transient energetic particle enhancement observed by Cluster is generated in the magnetic field pile-up region.

\section{Theory and models}

This section summarizes different theoretical approaches, modeling efforts and explanations of the physical processes relevant to the observations discussed in the previous section. From the plasma physics point of view, the transient and localized phenomena in the magnetotail relate also to processes which are kinetic (in the electron diffusion region in reconnection and the thin current sheet structure), two-fluid or Hall MHD (in the ion diffusion region) and MHD in a large part of the magnetosphere. Thus the mesoscale processes and the coupling among them can not be accomplished using a single model of the plasma. Since the present models and simula-

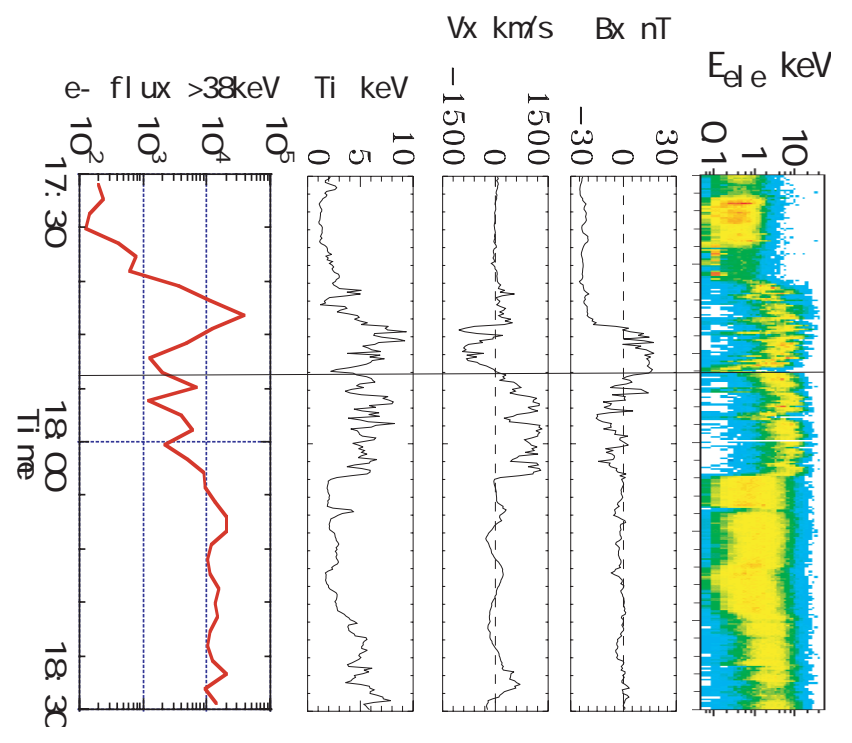

Fig. 26. The plasma sheet crossing data observed by Geotail on 10 December 1996. (Adopted from Hoshino et al., 2001).

tion codes are based on one of the plasma models, they have inherent limitations in providing an understanding the crossscale processes. In this section the theoretical efforts and models used to explain and interpret the observed transient and localized features are reviewed.

Although kinetic treatments are important in understanding collisionless reconnection and some current sheet instabilities, MHD models of reconnection and current sheet disturbances can be used to describe the signatures of the transient and localized processes (Sects. 3.1-3.8). On the other hand, the kinetic approach is essential to study the dynamics and acceleration of particles due to the reconnection and related processes, as discussed in Sects. 3.9 and 3.10. Global features of the magnetosphere, such as plasmoid formation, convection, etc., co-exist with ubiquitous multiscale features, often described in terms of power law dependences. A study based on the correlated data of the solar wind-magnetosphere interaction shows that the multiscale phenomena of the magnetosphere are not scale-free (Sect. 3.11).

The global MHD simulations (Lyon, 2000; Gombosi et al., 2003; Raeder, 2003) have provided a general framework for the understanding of the solar wind-magnetosphere coupling, in particular the connectivity among the differenet regions. In general such models have limitations in describing most of the localized and transient structures discussed in this review. In the case of the plasma bubbles (Sect. 3.6) the 3-D MHD model of the magnetotail (Birn et al., 2004) has provided an understanding of the plasma dynamics, and similar results have been obtained using the global MHD model (Shao et al., 2006). However, the global MHD simulations of substorms (Wiltberger et al., 2000), the thinning of the magnetail current yields thicknesses that are a few $R_{E}$, which is much wider than observed. 

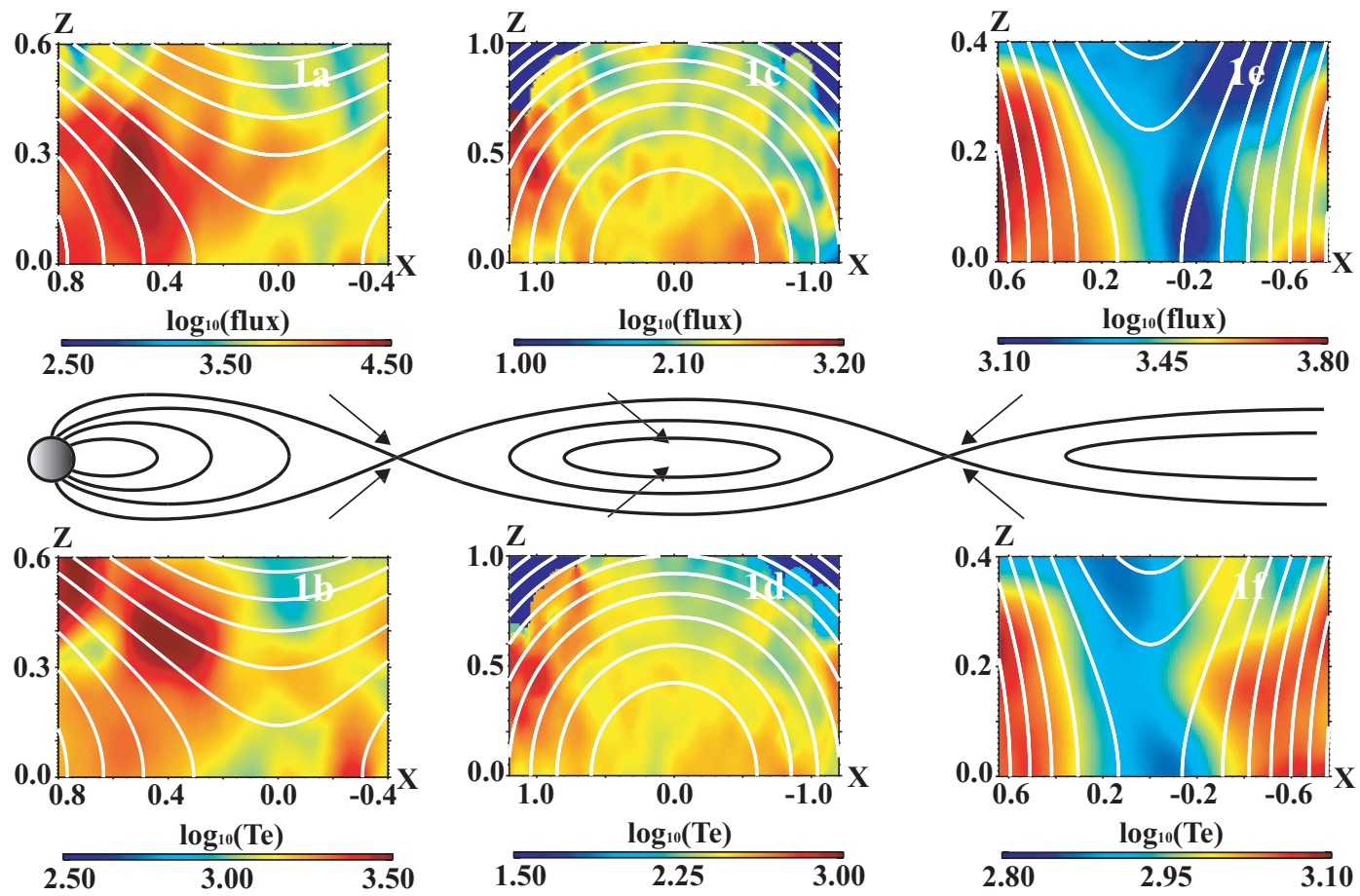

Fig. 27. Average profiles of the energetic electrons ( $>38 \mathrm{keV}$ ) (top) and temperature of the thermal electrons (eV) (bottom) observed in the near-Earth, the middle tail, and the distant tail. The cartoon of the magnetotail is shown in the middle panel. (From Imada et al., 2005).

\subsection{Analytical model of time-dependent reconnection}

The well-known Petschek's model of magnetic reconnection (Petschek, 1964) can be extended to incorporate a time varying reconnection rate, which can provide a basis for the interpretation of many transient phenomena. Such a model, illustrated in Fig. 28, involves a thin current sheet separating two different plasma and field regions (normal component $B_{z}$ is set to be zero). At some stage dissipation becomes important inside a small area, referred to as the diffusion region (panel a), where the reconnection is initiated. The localized initiation of reconnection and its subsequent evolution have the following features (Semenov et al., 2004b):

(i) Appearance of the reconnection electric field $E^{*}(t)$ in the diffusion region (its magnitude provides a measure of the reconnection rate).

(ii) Topological rearrangement of the magnetic field structure to form a region of reconnected flux tubes.

(iii) Generation of MHD waves propagating from the diffusion region.

(iv) Conversion of magnetic energy to plasma energy, mainly as propagating MHD waves.

(v) Convection of lobe plasma towards the current sheet and its rapid outflow along the current sheet (magnetic flux, plasma, energy, momentum and disturbances are transported from the inflow region to the outflow region).

(vi) Growth of the outflow region in size while it propagates along the current sheet produces perturbations in the inflow region (magnetic field compression above these structure plays important role in the overall energy balance).

At some stage the reconnection stops $\left(E^{*}\left(t_{0}\right)=0\right)$, when the outflow regions are detached from the site where the electric field was initiated, and propagate along the current sheet as solitary waves Fig. 28c.

Mathematically the time-varying reconnection model can be formulated as a solution of three closely-related problems (Semenov et al., 1983; Heyn and Semenov, 1996; Semenov et al., 2004a), as discussed below.

i. The Riemann Problem to identify the appropriate combination of non-linear MHD waves and specify the tangential $(\mathbf{v}, \mathbf{B})$ components in the outflow regions (Heyn et al., 1988). For the symmetric initial configuration of Fig. 28a, the following results for the plasma density $\rho$, gas pressure $p$, and the tangential plasma velocity component $v_{x}$ in the outflow region (Semenov et al., 1983; Biernat et al., 1987) are obtained:

$\rho=\rho_{0} \frac{\gamma(\beta+1)}{\gamma(\beta+1)-1}$

$v_{x}=v_{A}=B_{0} / \sqrt{\mu_{0} \rho_{0}}$,

where $\gamma$ is the polytropic index and $\beta$ is the plasma beta. These solutions do not depend on the reconnection rate, and therefore are time invariant.

ii. Surface wave analysis to determine the shape of the outflow region and the perpendicular $(\mathbf{v}, \mathbf{B})$ components 

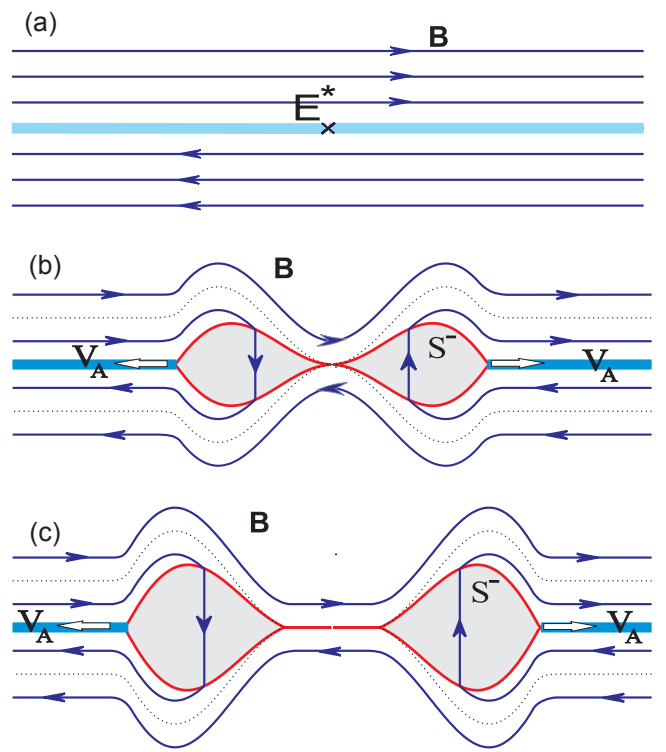

Fig. 28. Schematic illustrating the effects of time-varying reconnection in a symmetric geometry. In (a) reconnection is initiated through the appearance of a reconnection electric field in a localized part of the current sheet. In (b), during the switch-on phase, two outflow regions bounded by slow shocks $S^{-}$propagate along the current sheet away from the reconnection site. The structure of magnetic field and plasma flow in the vicinity of the X-line is very similar to the steady-state Petschek model (Petschek, 1964). In (c), the reconnection electric field has dropped to zero. During this switch-off phase, the outflow regions become detached from the reconnection site, but continue to move along the current sheet.

inside it from the linearized MHD equations, which are formally identical to the Kelvin-Helmholtz equation for surface waves (Heyn and Semenov, 1996). In addition to generating non-linear MHD waves (like those at the boundaries of outflow region) the reconnection also acts as a source for surface waves. For the symmetric case this yields (Semenov et al., 1983; Biernat et al., 1987)

$$
\begin{aligned}
& f(x)=\rho_{0} c / \rho v_{A} B_{0} x E^{*}\left(t-x / v_{A}\right) \\
& E(x, t)=E^{*}\left(t-x / v_{A}\right) \\
& B_{z}(x, t)=c / v_{A} E^{*}\left(t-x / v_{A}\right)
\end{aligned}
$$

where the function $f$ describes the shape of the slow shock and $E^{*}(t)$ is the assumed reconnection rate.

iii. External Disturbances. The two parts discussed above can be combined to provide the boundary conditions to compute the disturbances in the inflow region. The results (for example, for $\left.B_{z}(x, t)\right)$ can be presented as a convolution integral of the Green function $K(t, x, z)$ which consists of information about the initial current sheet, and the reconnection rate (Heyn and Semenov, 1996; Semenov et al., 2004a)

$B_{z}(x, z, t)=\int_{0}^{v_{A} t} K(t-\tau, x, z) E^{*}(\tau) d \tau$.

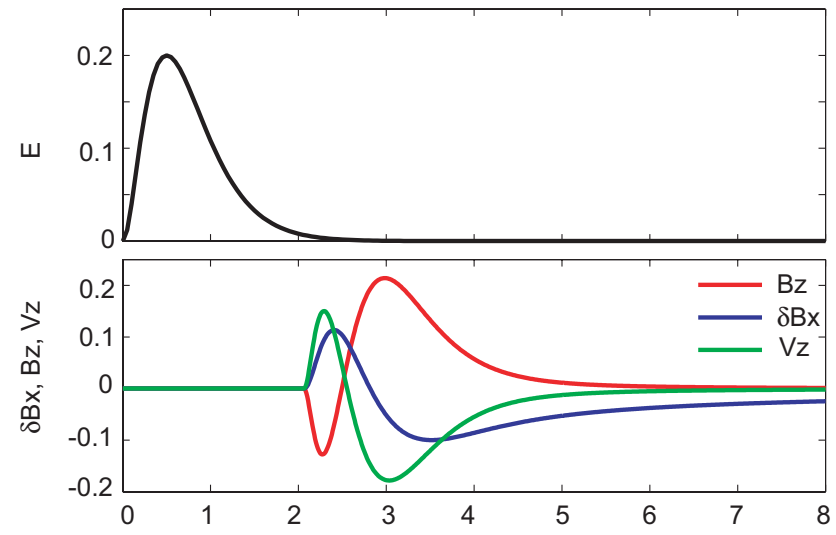

Fig. 29. Variations of the reconnection rate (top) and the corresponding disturbances of $\delta B_{x}=B_{x}-B_{0}, B_{z}$ and $v_{z}$ observed by an artificial spacecraft at the point $x=1, z=.5$ in the inflow region (bottom). Magnetic field is normalized to $B_{0}$, velocity to Alfvén velocity $v_{A}$, time to the duration of the pulse $t_{0}$, and distance to $v_{A} t_{0}$.

Predictions of the transient reconnection model. If the reconnection rate is specified we can compute the MHD perturbations in the inflow region. As an example, Fig. 29 shows variations of $B_{x}$ and $B_{z}$ components observed by an artificial spacecraft above the moving outflow regions. The $B_{z}$ component in the inflow region has a bipolar variation formed partly by the draping of magnetic field lines over the outflow region (Fig. 28b, c). The reconnection makes the $B_{z}$ variation asymmetric so that the positive pulse is bigger in comparison with the negative pulse for the geometry shown in Fig. 28. These features are similar to those frequently observed in the magnetotail (Sect. 2.4, Fig. 11). The propagating bulge-like outflow region also compresses the magnetic field (Fig. 29), and a combination of the $B_{x}-B_{z}$ variations is similar to the TCR signatures (Sect. 2.3, Figs. 6-8). The fast plasma inside the outflow region can be evidently identified with BBFs (Sect. 2.1). Thus the symmetric transient reconnection model reproduces the observed characteristics associated with BBFs, NFTEs and TCRs. Furthermore, it is evident that reconnection is inherently a current disruption process.

The disturbances $\left(B_{x}, B_{z}\right)$ in the inflow region are small, but a large region above them is affected, and therefore the total magnetic energy carried by TCR can be large (double the kinetic energy contained inside the outflow region, see Semenov et al., 2004b). The width in $z$ of the outflow region increases linearly with the distance from the $\mathrm{X}$-line. Close to the reconnection line it remains relatively small and $\left(B_{x}, B_{z}\right)$ variations are also small, while the amplitude of $\left(B_{x}, B_{z}\right)$ variations grow linearly with the distance from the $\mathrm{X}$-line even after the reconnection is switched-off. Hence NFTEs and TCRs will be observed only at some distance away from the X-line. 

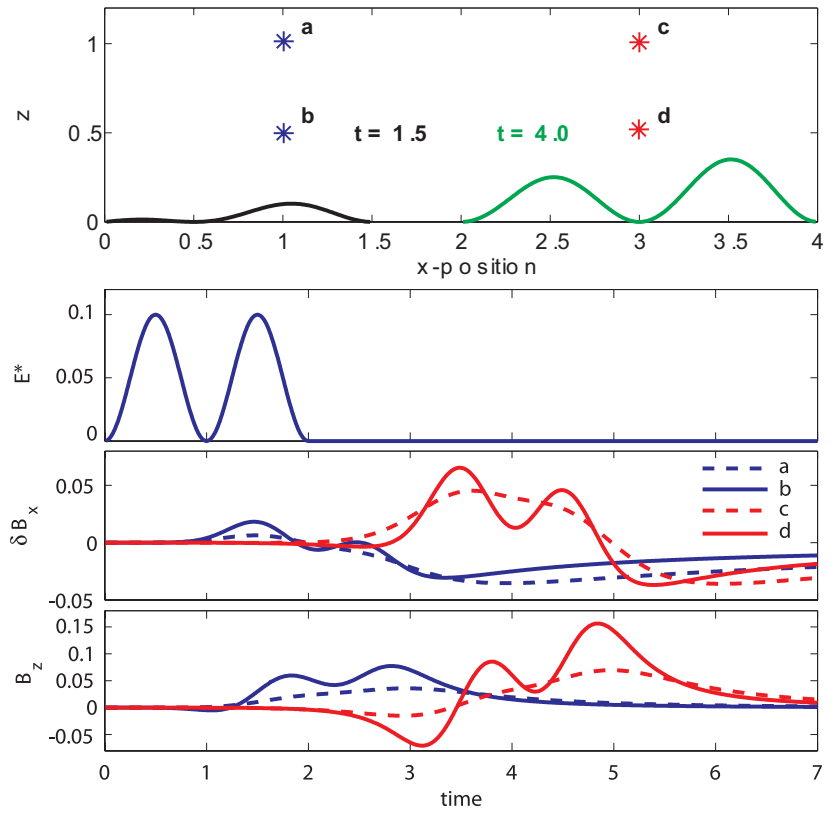

Fig. 30. Positions of artificial spacecraft a,b,c,d with respect to the outflow region (upper panel) produced by a double-pulsed reconnection rate (second panel). Corresponding disturbances of $\delta B_{x}$ and $B_{z}$ observed by the artificial spacecraft are shown in the two lower panels. The magnetic field is normalized to $B_{0}$, the velocity to the Alfvén velocity $v_{A}$, the time to the duration of the pulse $t_{0}$, and the distance to $v_{A} t_{0}$.

The resulting spatial variations are illustrated in Fig. 30, which compares the perturbations in the inflow region at different distances both along and across the current sheet. In contrast to Fig. 29, the reconnection rate is chosen here to be of double-pulse shape. This reveals how strongly the shape of magnetic perturbations depends on $Z$ : the details are smeared out at large $Z$, where the double-pulse origin of the perturbations is hard to distinguish. The important conclusion from Fig. 30 is how variable the waveforms in the inflow region are, even in simplest model and for simple reconnection rate behavior. This may provide a natural explanation for the different types of waveforms in the inflow region, including these shown in Sects. 2.1, 2.3, 2.4.

Reconstruction of reconnection rate: the inverse problem. The Eq. (6) can also be used as an integral equation to determine the reconnection rate $E^{*}(t)$ from the time series of magnetic field perturbations observed by a spacecraft in the inflow region. In the Laplace space it has the form of simple algebraic equation $B_{z}(p)=K(p) E(p)$, which is solved using the standard regularization theory. Thus, the reconnection rate $E^{*}(t)$ and also the position of the spacecraft relative to the X-line can be computed (Semenov et al., 2005).

As an illustration of this method, Fig. 31 shows the reconstruction results for a near-Earth reconnection event in which the Cluster $\mathrm{C} 2$ spacecraft was in the tailward outflow region, while the other spacecraft $(\mathrm{C} 1, \mathrm{C} 3, \mathrm{C} 4)$ were in the inflow

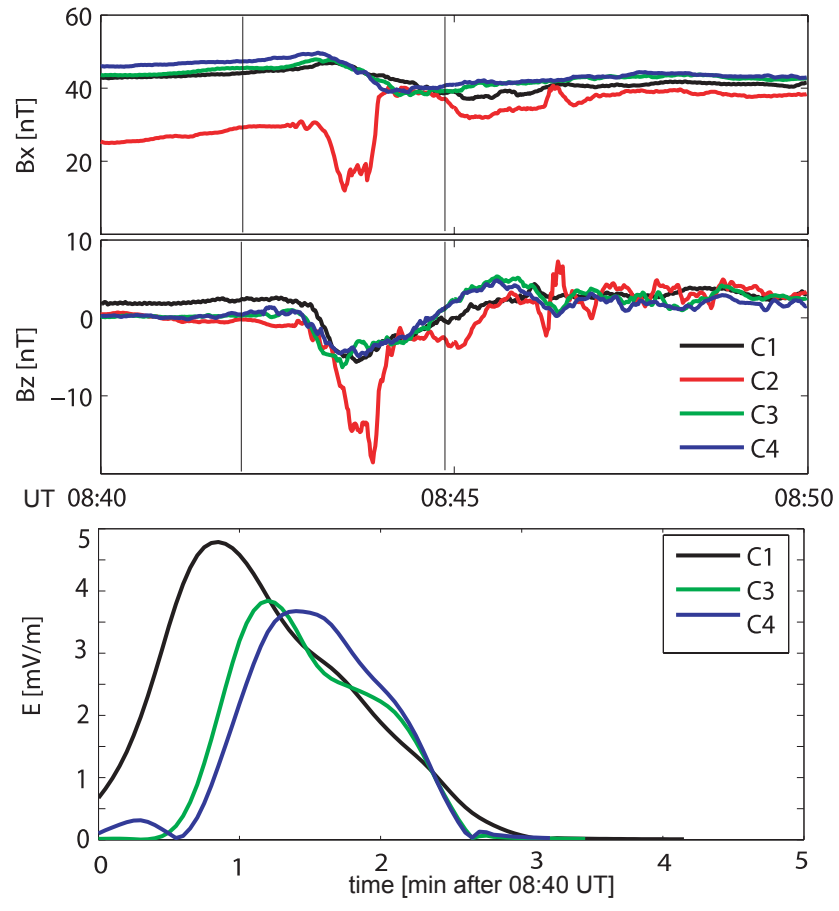

Fig. 31. The reconnection electric field reconstructed from Cluster observations on 26 September 2005.

region. The peak of the reconstructed reconnection rate was $3.7-4.8 \mathrm{mV} / \mathrm{m}$ and the pulse duration was from 2 to $3 \mathrm{~min}$ (Fig. 31). The reconstructed location of the $\mathrm{X}$-line recovered from the data was at $X=-9.3 R_{E}$ for $\mathrm{C} 1,-8.8 R_{E}$ for $\mathrm{C} 3$ and -11.3 for $\mathrm{C} 4$.

The simplest, symmetric 2-D model used here does not include a number of important effects, e.g., the finite thickness of the current sheet, the north-south asymmetry, the finite length of the reconnection line. Moreover the guide $B_{y}$ component and the normal $B_{z}$ component are set to be zero. Inclusion of these effects leads to a number of new effects. Most important among these are that the NS asymmetry and $B_{y}$ component lead to different speeds (or directions, in case of $B_{y}$ ) of the waves propagating in the two half-spaces and thus to tilts of the neutral sheet threaded by reconnected flux tubes. Some north-south sheet motions resembling flapping motions (Sect. 2.7) may also appear (see Sect. 3.2 for further discussion of some $B_{y}$ effects).

\subsection{Localized reconnection}

In another model of reconnection, the effects of an initial current sheet of finite thickness and non-zero guide field $B_{y}$ are included. While the time-dependent reconnection (Sect. 3.1) cannot produce negative $B_{z}$ at the exact center of the current sheet, reconnection localized in the dawn-dusk direction, in the presence of non-zero $B_{y}$, can reproduce such a $B_{z}$ perturbation at the leading edge of Earthward jets, such as those discussed in Sect. 2.2. 
Shirataka et al. (2006) studied the 3-D structural evolution resulting from reconnection in the tail, by conducting 3-D MHD simulations in which a non-zero, uniform $B_{y}$ component is initially present. For triggering the reconnection onset, an ad-hoc anomalous resistivity with a finite $y$ extent at a fixed $x$ position in the current sheet $(z=0)$ was used. In this situation, a pair of Earthward and tailward reconnection jets with finite widths emanates from the resistive region. Above and below the current sheet, there is no obstacle (pre-existing plasma in the sheet) to the Earthward jets. Therefore, hot plasma generated by reconnection streams along the reconnected field lines at a speed higher than that of the jet near the $z=0$ plane, where the pre-existing plasma blocks the Earthward jet. Consequently, two field-aligned jets of hot plasmas are produced, each above and below the plasma sheet. In the presence of a non-zero guide field, for example under positive $B_{y}$, the field-aligned jets above and below the plasma sheet are brought duskward and dawnward, respectively. In other words, the pair of the field-aligned jets becomes tilted with respect to the $z$ axis (Fig. 32a). The flux tubes filled with hot plasma have such an excess pressure that they expand in the $y-z$ plane. This expansion proceeds northward (southward) at $y<0(y>0)$ and twists the magnetic field lines embedded in the plasma sheet ahead of the jet, which are initially oriented purely in the dawn-dusk direction. Associated upward and downward shifts of the current sheet can be a potential mechanism for the current sheet flapping, discussed in Sect. 2.7. The bending of field lines develops as the Earthward jet comes into contact with the pre-existing plasma sheet, and eventually produces a southward $B_{z}$ component in regions immediately Earthward of the jet in the $z=0$ plane. On the other hand, a northward $B_{z}$ region is generated in the jet itself, owing to the pile-up of reconnected field lines onto the pre-existing plasma sheet. Since this southwardthen-northward $B_{z}$ structure propagates Earthward with the jet, it can be recorded by a spacecraft situated in the plasma sheet as a bipolar $B_{z}$ signature.

An important aspect of this model is that a single reconnection X-line is sufficient to produce the bipolar magnetic signature, contrary to the model proposed by Slavin et al. (2003b) that requires multiple X-lines (Sect. 3.3). Here the prerequisites for the negative $B_{z}$ to appear at the leading part of reconnection jets are: (i) a non-zero guide field in the middle of the current sheet, which eventually gets twisted to produce the negative $B_{z}$; (ii) a finite extent of the reconnection region, which generates narrow (3-D) jets; and (iii) a nonzero guide field in the lobe region, which leads to the tilt in the $(Y, Z)$ plane of the two field-aligned jets.

Figure $32 \mathrm{~b}$ shows time series data of $B_{y}, B_{z}$, number density, and the $x$ component of the flow velocity, recorded by an artificial spacecraft situated at 11.25 $R_{E}$ Earthward of the reconnection (anomalous resistivity) region. The initial $B_{y}$ and the width of the resistivity region are set to be $4 \mathrm{nT}$ and $3 R_{E}$, respectively, based on earlier observations of BBFs (Angelopoulos et al., 1997, see also Sect. 2.1). The virtual (a)

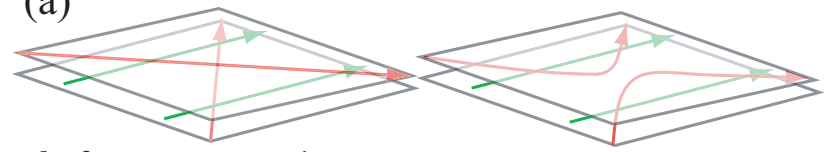

before reconnection after reconnection
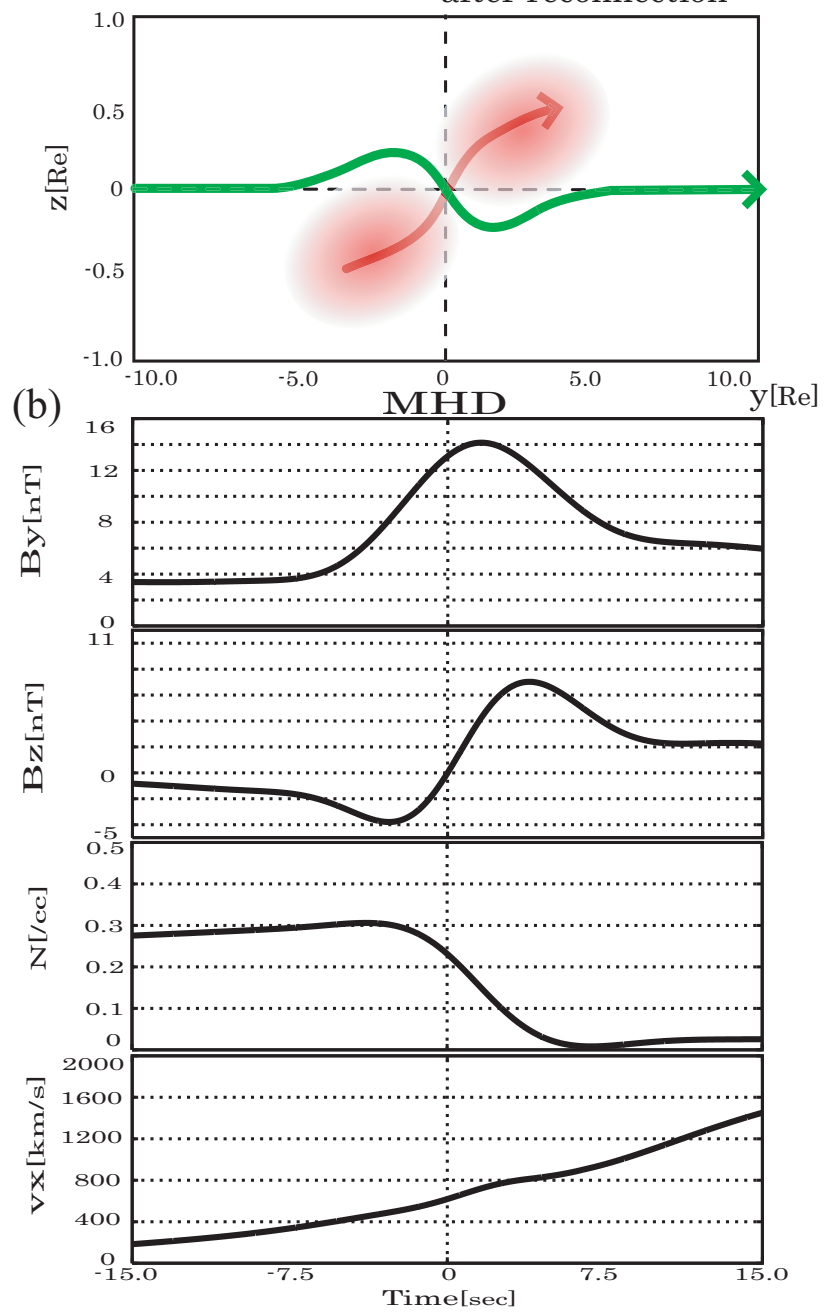

Fig. 32. (a) Schematic showing how negative $B_{z}$ can be produced at the jet leading edge. The red and green lines represent reconnected and current sheet embedded field lines, respectively. (b) Observation by a virtual satellite situated at the current sheet center. The result is from an MHD simulation with the guide field $B_{y 0}=4 \mathrm{nT}$ and the dawn-dusk extent of anomalous resistivity region of $3 R_{E}$. The initial half-thickness of the current sheet is set to be $0.5 R_{E}$. Note that the bipolar $B_{z}$ signature is reproduced, without invoking multiple reconnection X-lines. (From Shirataka et al., 2006).

spacecraft observation demonstrates that a southward-thennorthward $B_{z}$ perturbation occurs exactly when the speed of the Earthward flow increases. The time $T=0$ is defined here as the moment when $B_{z}$ changes its polarity from southward to northward. There is a finite $B_{y}$ component before the arrival of the jet, representing the initial guide-field, and this $B_{y}$ 


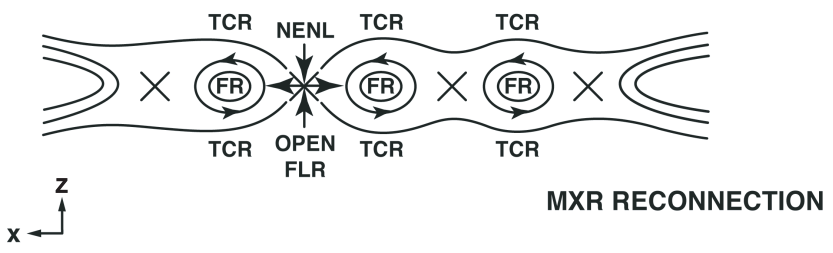

Fig. 33. Schematic representations of flux rope and TCR formation under the action of multiple X-line formation. (Adopted from Slavin et al., 2003b).

component peaks in the middle of the bipolar perturbation. A density peak occurs prior to the center of the bipolar signature. The flow velocity $V_{x}$ increases monotonically throughout the interval shown. It is seen therefore that the simulation reproduces all the essential features seen in the observations (Slavin et al., 2003b, see also Sect. 2.2 and Fig. 9). The peak in $B_{y}$ at $T \sim 0$ is due to the compression of the guide field by the convergence of $V_{x}$ at the front edge of the jet. The peak in density slightly earlier than the $B_{y}$ peak is due to the compression of the pre-existing plasma-sheet plasma by the jet. The time shift between the two peaks occurs because the initial $B_{y}$ is uniform throughout the simulation domain whereas the density is substantially higher in the plasma sheet than in the lobe region. This shift, seen in the observations as well, is what this localized reconnection model can reproduce but the multiple X-line reconnection model cannot (Ohtani et al., 2004).

Shirataka et al. (2006) have also shown that a profile similar to that shown in Fig. 32b is reproducible by Hall-MHD simulations as well, indicating that even in the situation where ion inertia effects may play a role, as in the tail, the bipolar magnetic structure at the leading edge of the jet can be generated as long as a non-zero guide field is present and the jet width is finite. It should be pointed out that the width of the reconnection jets, the guide-field intensity, and the magnitude of southward $B_{z}$ at the jet front edge are mutually related. Their parameter survey showed that the southward peak in $B_{z}$ increases as the guide field is intensified and as the reconnection jets become narrower. Such a relationship may be verified in future by a statistical study, although achieving this requires accurate estimation from measurements of the above three parameters, including the jet width that may be determined only by coordinated multi-point measurements (Nakamura et al., 2004a). Another remarkable feature is that, at $z \sim 0$, the bipolar $B_{z}$ signature occurs in the central part of the jets, but is not seen or is very weak at the dawn and dusk flanks of the jets. Confirmation of this may be possible on an event basis by Cluster observations, when the spacecraft located in the exact vicinity of the center of the plasma sheet are separated by a few $R_{E}$ in the dawn-dusk direction.

\subsection{Multiple X-lines}

When reconnection takes place at two or more X-lines simultaneously it is termed "multiple X-line reconnection" or "MXR" reconnection (Lee, 1995; Lee and Fu, 1985). Initially, such reconnection in the plasma sheet involves only closed plasma sheet flux tubes. The Alfvén speed is low at the center of the cross-tail current sheet so that the reconnection will proceed slowly and the outflow from the X-lines will be only $\sim 100 \mathrm{~km} / \mathrm{s}$, which is insufficient to cause significant dynamical interaction between the X-lines. However, as first noted by Schindler (1974), one of the X-lines will inevitably outpace the others in a nonlinear manner and begin to reconnect first the flux tubes in the outer plasma sheet and, finally, the lobe flux tubes where the Alfvén speed is typically several thousand $\mathrm{km} / \mathrm{s}$ (see Hesse et al., 1996). At that point, everything (X-lines with lower reconnection rates and any flux ropes formed between them) Earthward of the first X-line will be carried toward the Earth, and all material tailward of that point will be rapidly swept down the tail. Hence, the formation of flux ropes by MXR is in a sense only a preliminary event to the open flux reconnection at a single dominant neutral line in the NENL model of substorms (e.g., Baker et al., 1996). The existence of MXR is now supported not only by numerical simulations (Ohtani et al., 2004; Shay et al., 2003; Drake et al., 2006), but also by the recent observation of a multiple X-line event by Cluster (Owen et al., 2005; Eastwood et al., 2005). The occurrence of MXR may also provide the multiple sources needed to explain the TDIS signatures discussed in Sect. 2.9.

It was suggested by Slavin et al. (2003c) that the physical properties of the Cluster TCRs are consistent with their being caused by the rapid motion of multiple, small-scale flux ropes recently discovered in the plasma sheet using Geotail (Slavin et al., 2003b) and Cluster (Slavin et al., 2003a; Zong et al., 2004) observations. These observations can thus be interpreted in terms of the MXR model for the break-up of the near-Earth current sheet.

Figure 33 illustrates how small flux ropes may form due to reconnection at such multiple $\mathrm{X}$-lines. In this figure, $4 \mathrm{X}$ lines $(X)$ are depicted, and create 3 closed loop plasmoids or helical flux ropes (FR). The first of these X-lines to begin to reconnect open magnetic flux in the lobes becomes the near-Earth neutral line (NENL). Open field line reconnection at this X-line will subsequently sweep the other X-lines and associated flux ropes Earthward and tailward in its reconnection outflow jets, thus creating the TCR signatures in the exterior near-tail lobes (Lee and Fu, 1985; Elphic et al., 1986; Moldwin and Hughes, 1994; Slavin et al., 2003a).

However, we note that these near-tail TCRs could also be produced by highly-time dependent, "impulsive" reconnection, as suggested by Sergeev et al. $(1987,1992,2005)$ and described in Sect. 3.1. Definitive testing of these various hypotheses requires further multi-point measurements that provide simultaneous observations of both the lobe compression 
region and the fast-moving hot plasma bubble or flux rope in the central plasma sheet. Separations in the north - south direction of several times $10^{4} \mathrm{~km}$ will be necessary for these studies and may become available during the next few years of the Cluster extended mission.

\subsection{Reconstruction of equilibrium magnetic structures}

Magnetic field structures in equilibria, such as flux ropes in a relaxed state (Sect. 2.2), can be reconstructed from in situ measurements. The method is called Grad-Shafranov (GS) reconstruction (Sonnerup et al., 2006), and its basic version (Hau and Sonnerup, 1999) assumes that (i) the structure to be reconstructed is $2-\mathrm{D}$, i.e., is elongated in some direction, $z$, called the invariant axis, (ii) it is time independent, when seen in a co-moving frame, and (iii) inertia effects are negligible. Then, the force balance equation describing magnetohydrostatic equilibria $\mathbf{j} \times \mathbf{B}=\nabla p$ can be reduced to the so-called GS equation:

$\frac{\partial^{2} A}{\partial x^{2}}+\frac{\partial^{2} A}{\partial y^{2}}=-\mu_{0} \frac{d P_{t}}{d A}$

where $A$ is the partial vector potential, $A(x, y)$, and $P_{t}$ is the transverse pressure, $P_{t}=\left(p+B_{z}^{2} /\left(2 \mu_{0}\right)\right)$. The field lines projected onto the reconstruction $(x-y)$ plane are represented as equi- $A$ contour lines, and the axial field, $B_{z}$, as well as the plasma pressure, $p$, become functions of $A$ only.

Since, in contrast to the reconstruction of reconnection rate presented in Sect. 3.1, time independence of the structure is assumed, time series of data seen by spacecraft are interpreted as being due to spatial structures convected past the spacecraft. The GS Eq. (7) can then be solved as a spatial initial value (Cauchy) problem, using combined plasma and field measurements, and we obtain a 2-D map of $A, A(x, y)$. Moreover, when multi-point data from Cluster are available, a single optimal field map can be generated based on four independent field maps, each produced for each satellite (see Hasegawa et al., 2005, for details). The GS technique has been applied mostly to the magnetopause equilibrium, and it can be applied to the case of the magnetotail. In the case of the former, this techniques has been applied extensively to the the flux transfer events (FTEs), which are similar to the flux ropes and TCRs in the latter. In the following two cases associated with Earthward flows in the magnetotail are discussed.

Figure 34 shows a reconstruction result for $\mathrm{a}-/+B_{z}$ perturbation seen by Cluster during an Earthward flow. Since $\mathrm{C} 3$ was in the vicinity of the current sheet center while the other three $(\mathrm{C} 1, \mathrm{C} 2$, and $\mathrm{C} 4)$ were a few thousands $\mathrm{km}$ north of it, the entire core part of the structure was reasonably reconstructed. A circular flux rope having a diameter of $\sim 2 R_{E}$ is clearly seen in the reconstructed map. The invariant $(z)$ axis is determined to be approximately in the GSE $y$ direction. It is thus suggested that multiple $\mathrm{X}$-line reconnection

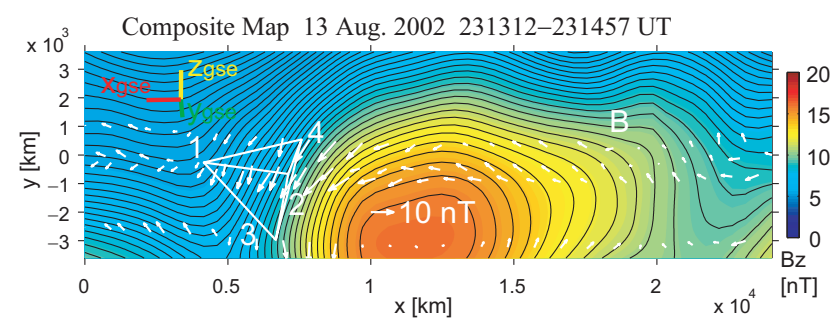

Fig. 34. Magnetic field map reconstructed from Cluster observations of a bipolar $-/+B_{z}$ signature on 13 August 2002, 23:13:1223:14:57 UT. Black lines show magnetic field lines transverse to the invariant $(z)$ axis, while colors show the out-of-plane field component. Measured field vectors are represented by white arrows and projected onto the reconstruction $(x-y)$ plane along the four spacecraft paths. The spacecraft move from left to right in the map. The optimally selected GS axes are in GSE: $x=(-0.998,0.052,0.019)$, $y=(-0.018,-0.635,0.773)$, and $z=(0.052,0.771,0.635)$.

(Sect. 3.3) was responsible for the creation of the $B_{z}$ structure in this event.

A similar observation during a BBF, but when Cluster was somewhat away from the current sheet center was presented by Snekvik et al. (2007). In this case, the reconstruction results (Fig. 35) show that the invariant axis was oriented roughly in the GSM $x$ direction, and that an intense Earthward-directed FAC filament was present while Cluster was engulfed in the BBF (right part of the maps, see also Fig. 12 and Sect. 2.5 in which the same event is discussed). It was therefore concluded that Cluster encountered the dawnside part of an FAC pair, formed presumably in association with the BBF.

As demonstrated here, the GS maps provide information about the shape, dimension, orientation, and magnetic topology of observed local structures, thus helping us to infer which mechanism is responsible for the localized structures. However, recent experiments using synthetic data from 3-D MHD simulations of localized reconnection (Sect. 3.2) show that on occasion, the GS method erroneously produces a flux rope-type (closed field loop) structure that does not in fact exist in the simulation. The result indicates the need to be cautious about interpretation of results, especially when data analysis techniques are based on some models and/or assumptions.

\subsection{Hall currents and shocks in reconnection regions}

The occurrence of Hall currents near a reconnection site has long been recognized (Sonnerup, 1979; Terasawa, 1983). These currents are expected to induce a quadrupole configuration of the magnetic-field component, $B_{y}$, transverse to the plane in which the main reconnection effect is seen (in the tail, typically the GSM $x-z$ plane). The presence of these quadrupole fields, and of the current loops responsible for them, have subsequently been verified in many numerical 

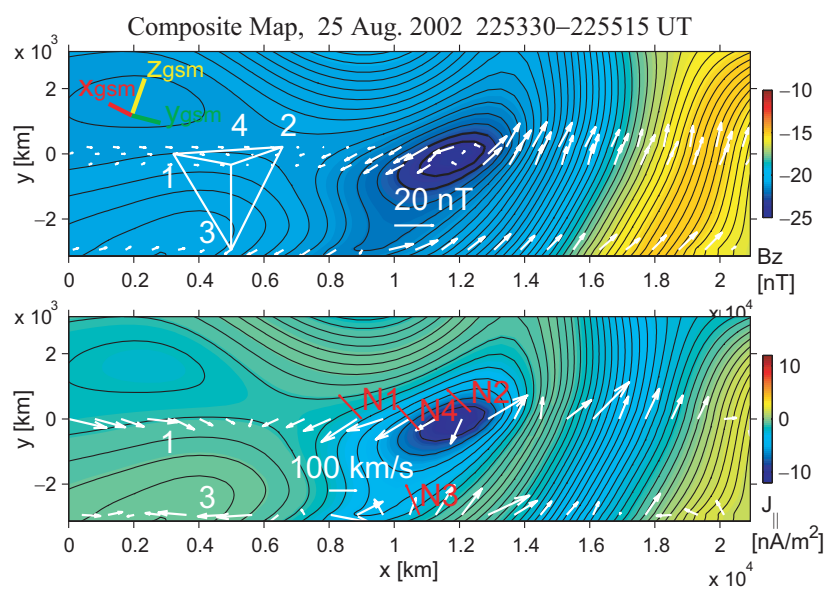

Fig. 35. Field maps for an FAC filament at the dawn flank of a BBF encountered by Cluster. Colors in the top and bottom panels show the out-of-plane field component $B_{z}$ and field-aligned current estimated from the recovered fields, respectively (see Snekvik et al., 2007, for details). White arrows represent measured fields and velocities in the top and bottom, respectively. Note that the axes $x, y$ and $z$ here are not in GSM, but in the GS reconstruction coordinates. The GS axes are in GSM: $x=(-0.585,0.737,0.338)$, $y=(0.295,-0.195,0.936)$, and $z=(0.755,0.647,-0.103)$.

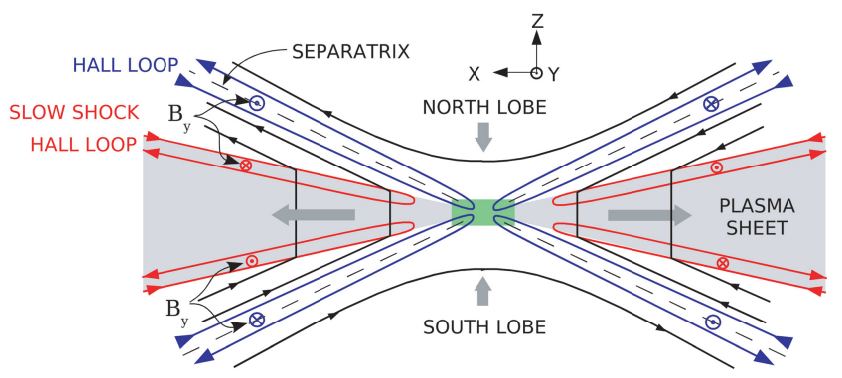

Fig. 36. Schematic drawing of Hall-current driven loops in the region surrounding a reconnection site (separatrix loops in blue; slow shock loops in red). Green box in the center is the ion "diffusion" region.

simulations (e.g., Hesse and Winske, 1994; Shay et al., 1999) and, more recently, also in spacecraft data taken near reconnection sites in the geomagnetic tail. Øieroset et al. (2001) used Wind data at $x \simeq-60 R_{E}$ to demonstrate the presence of the out-of-plane quadrupole fields within the ion diffusion region, with a magnitude of about $40 \%$ of the reconnecting field; Nagai et al. $(1998,2001,2003)$ used Geotail data at $x \simeq-25 R_{E}$ and somewhat further from the reconnection site to show their presence with an amplitude of about $20 \%$. Runov et al. (2003a) used Cluster observations to identify the quadropole field near a reconnection site. They found peak amplitudes of $50 \%$ or more and were able to identify a characteristic width comparable to the ion inertial scale. These values are roughly consistent with simulation results (e.g., Nagai et al., 2003).
In discussions of collision-less reconnection, the Hall effect is often couched in terms of electron-whistler dynamics (e.g., Shay et al., 1999) and appears to play an important role in allowing reconnection to occur at rates comparable to those predicted by Petschek (1964). The GEM magnetic reconnection challenge (Birn et al., 2001) examined the reconnection rate for a simple Harris equilibrium under a specified set of initial conditions using different simulation codes, viz. MHD, Hall MHD, hybrid and full particle codes. These simulations show that the codes that include the Hall effect produce fast reconnection and the rates are indistinguishable. In the absence of the Hall term in the generalized Ohm's law, the much slower rates of the Sweet-Parker model (Parker, 1957) of reconnection are observed. The mechanism whereby the Hall term allows fast reconnection to occur is not fully understood; a localized patch of enhanced resistivity, or localized non-isotropic pressure, may also lead to fast reconnection.

Away from the reconnection site, Petschek's model predicts the presence of two pairs of slow-mode shocks, which accelerate the incoming plasma to form two outflow jets pointing along $\pm x$, away from the reconnection site. These shocks are near the switch-off limit so that the outflow in the jets is approximately Alfvénic. In the presence of the Hall term in Ohm's law, the internal structure of these shocks exhibits the polarization of the ion-whistler mode (Coroniti, 1971), an effect that should be observable in the tail but, to date, has not been identified and discussed. Spacecraft passages through the slow shocks themselves have been reported from ISEE-3 data in the deep tail (Feldman et al., 1984, 1987) and from Cluster data at $x \simeq-19 R_{E}$ (Eriksson et al., 2004). If both the electron- and the ion-whistler polarizations are present at $x$-values well removed from that of the reconnection site, one would expect the out-of plane component (the GSM $y$-component) to display an overall octupole behavior around the site. Such behavior has not been reported to date.

A schematic drawing of a possible magnetic field and current configuration in the GSM $x-z$ plane and in the vicinity of a reconnection site is shown in Fig. 36. The first set of current loops encountered by the plasma as it flows toward the reconnection site from above or below can be understood as driven by differential motion, in the $\pm z$-direction, of electrons and ions. As the $x$-axis is approached from above or below, near the reconnection site (in what is often called the diffusion region), the ions are stopped over a distance comparable to the ion inertial length, whereas the electrons continue their motion until they come within an electron inertial length of the $x$-axis. The resulting currents in the $\pm z$-direction are closed by nearly field-aligned currents that appear to flow around the separatrices (Nagai et al., 2003) toward and away from the reconnection site, as shown schematically in the figure. Upstream of the separatrices, the currents are caused by a nearly field-aligned beam of low energy electrons flowing toward the reconnection site (Fujimoto et al., 2001; Øieroset et al., 2001; Nagai et al., 2003). Presumably they are pulled in to 
maintain charge neutrality in the ion diffusion region. They also drift in the reconnection electric field, $E_{y}$, toward and across the separatrices. The current branches downstream of the separatrices are associated with higher-energy electron beams leaving the reconnection site after having been accelerated there (Nagai et al., 2003) and, at the same time drifting towards $z=0$ in the reconnection electric field. Thus the model accommodates flow across the separatrices, as required during reconnection (Vasyliunas, 1975). Since the magnetic-field component in the direction perpendicular to a separatrix surface is zero at the surface itself but reverses sign across it, both branches of a Hall-driven current loop can be interpreted as electron-whistler waves standing in the flow. The upstream waves rotate the magnetic field to generate the quadrupole field component, $B_{y}$, and the downstream waves then rotate the field back to the $x-y$ plane. As discussed in Sect. 2.5, these current loops appear to extend to large distances from the reconnection site (Fujimoto et al., 2001) and, on the Earthward side, may actually close in the ionosphere, where their signatures may have been observed (Nagai et al., 2003; Treumann et al., 2006). On the tailward side, the extent of these currents could be limited by the presence of plasmoids or other reconnection sites.

Closer to the $x$-axis and somewhat further away from the diffusion region, another set of current loops is expected, as shown in Fig. 36. These loops form part of the slowmode shocks in Petschek's model. Their magnetic structure for isotropic pressure is illustrated in Fig. 37a, which shows the rotation of the magnetic field tangential to the shock surface as one travels with the plasma across the shock. The shock has an ion-whistler wave train attached to its downstream side. Its spatial extent and amplitude depend on the ratio of ion inertial length to resistive length, as illustrated in Fig. 37b. For small resistive lengths, the wave trains from the two shocks on opposite sides of the $x$-axis should intermingle to create a complicated out-of-plane magnetic field in the exit plasma jets, which are sandwiched between the Northern and Southern Hemisphere slow shocks. This effect has not been reported. Note however that there are circumstances, involving non-isotropic pressure, where the slow-mode shock can have a standing electron-whistler wave train attached to its upstream side (Walthour et al., 1994). Another unanswered question is how near the reconnection site the slow shocks will form, and how far from it they will extend. On the Earthward side, the inner magnetosphere forms an obstacle that will slow and deflect the Earthward jet. The deceleration could perhaps be accomplished in a fast-mode shock sitting across the jet (Yang and Sonnerup, 1976). The slow shocks would terminate at this location. In fact, the slow shocks are seldom seen at all on the Earthward side of a near-Earth reconnection site. On the tailward side, they are more frequently seen and could in principle extend to large distances. However they could also be terminated by the presence of plasmoids and additional reconnection sites.
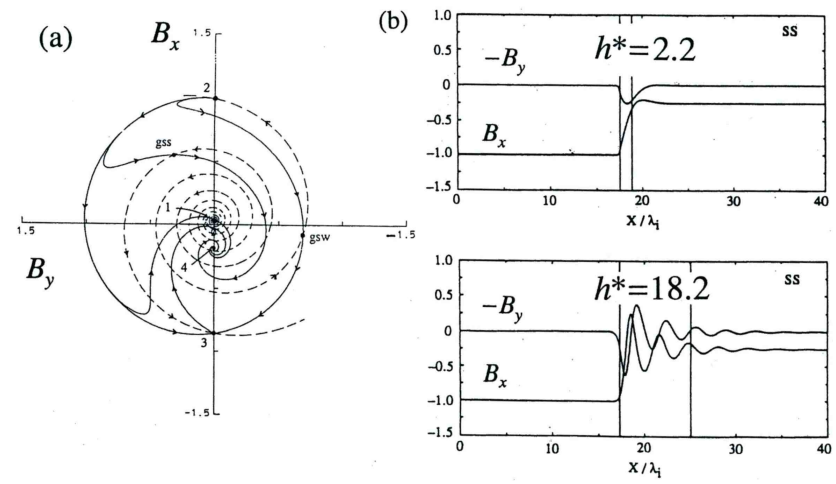

Fig. 37. Magnetic structure of shocks in Hall MHD. (a) Example showing the behavior of the magnetic field components in the shock plane (compare to lower left shock in Fig. 36) for small resistivity. The slow-shock upstream and downstream states are at points 3 and 4, respectively (also, fast shock: $1 \rightarrow 2$; intermediate shocks: $1 \rightarrow 3,2 \rightarrow 3,1 \rightarrow 4,2 \rightarrow 4$ ). The magnetic-field and flow components across the shock are both directed out of the plane of the paper (along $+\hat{z}$ ). (From Hau and Sonnerup, 1990). (b) Spatial profiles of the field components, $B_{x}$ and $B_{y}$, tangential to a slow shock (compare to lower left shock in Fig. 36) for $h^{*}=2.2$ and $h^{*}=18.2$, where $h^{*}$ is the ratio of ion-inertial length, $\lambda_{i}$, to resistive length, $\lambda_{r}$. Upstream parameter values are: Alfvén number $A_{3}=0.91$; shock angle $\theta_{3}=85^{\circ}$; plasma pressure to magnetic pressure $\beta_{3}=0.04$. (From Hau and Sonnerup, 1992).

In summary, the existence of the Hall-current produced quadrupole fields near the separatrices appears well established, whereas the out-of-plane fields associated with the slow shocks have, to our knowledge, not been reported to date. On the whole, the schematic in Fig. 36 should be a useful tool in the continued interpretation of reconnectionassociated phenomena in the geomagnetic tail.

\subsection{Bubble}

The formation of plasma bubbles is inevitable inside the large region of closed flux tubes in the magnetotail through which the plasma is transported to the inner magnetosphere. In the ideal slow-flow MHD theory the integral quantity $p V^{5 / 3}$, where $V$ is the volume of a magnetic flux tube, is conserved along the plasma flow streamline during adiabatic plasma transport. This quantity, $S=p V^{\gamma}$, is often referred to as the entropy parameter, (e.g., Wolf et al., 2006) and recent MHD and PIC simulations for a particular case of 2-D reconnection have shown that it is conserved (Birn et al., 2006). This entropy plays an important role in the MHD stability of closed magnetic configurations. In particular, the tail-like closed magnetic configuration (with negligible loss through the ends of flux tubes) is stable against the interchange instability if $S$ increases downtail (i.e. $d / d r\left(p V^{\gamma}\right)>0$ ). Validity of this condition for the Earth's magnetotail was verified by computations based on empirical magnetospheric models (Garner et al., 2003). However, this also implies that 2-D Earthward 

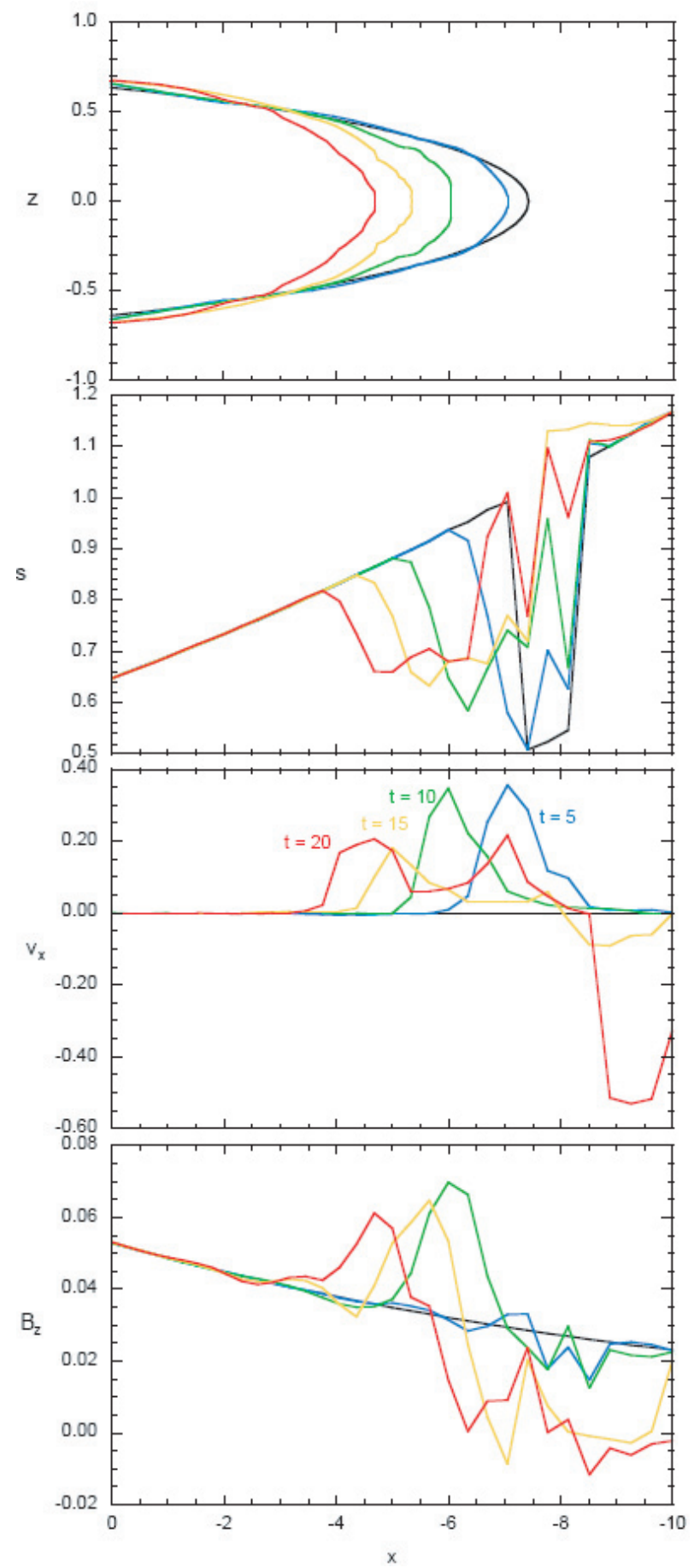

Fig. 38. Evolution of plasma-depleted tube with time in the numerical simulation of a plasma bubble. The bubble is easier to identify based on the depletion the entropy function $s=p^{1 / \gamma} / \rho$ The upper panel shows the motion and reconfiguration of flux tube at the inner edge of the bubble. Other panels show the evolution of entropy, flow velocity and magnetic field $B_{z}$-component in the equatorial plane. $\mathrm{T}=20$ corresponds to approximately $8 \mathrm{~min}$ of real time. (From Birn et al., 2004). convection is prohibited (suppressed), providing a so-called convection crisis (Erickson and Wolf, 1980): The magnetic configuration becomes progressively more stretched, providing the magnetic energy storage in the tail. This is a common view of how the substorm growth phase works. However, the situation changes if some process creates a plasma inhomogeneity, thus changing the entropy in the localized flux tube. This makes the plasma configuration interchange-unstable, with the localized plasma-depleted tube (called plasma bubble) moving Earthward, into the region of smaller $S$ and stronger magnetic field.

This problem was theoretically analyzed the for tail-like magnetic configuration using a thin filament MHD approximation (Chen and Wolf, 1999) or numerical simulations of localized bubble dynamics using a 3-D MHD code (Birn et al., 2004). These studies led to the following scenario, depicted in Fig. 38. Created by a depletion in plasma pressure (density or temperature), this localized underpopulated tube gets compressed to reach an equilibrium with the surrounding media in a short time $(\sim 1 \mathrm{~min})$. Both the depleted pressure and increased $B$ ( $B_{z}$ in the equatorial region) contribute to the low value of the entropy $S_{B}$ inside the bubble. After a redistribution of the plasma and fields over the bubble, it has stronger $B_{z}$, smaller $S_{B}$ and becomes more dipolar in shape compared to the neighboring plasma tubes. The enhanced $\mathbf{j} \times \mathbf{B}$ force drives the bubble Earthward as long as its entropy $S_{B}$ is less than $S_{0}(r)$, the entropy of the background plasma, which decreases Earthward. In other words, because of the depressed cross-tail current (less current carriers, stronger $B)$ the bubble is polarized, with negative (positive) electric charges on its dusk (dawn) flanks, which in turn implies a shear motion of plasma within the bubble with respect to the surrounding medium, as depicted in Figs. 39 and 40. The inhomogeneity inside the bubble causes the differential motions and deforms it, independently of initial cross-sectional shape, into an elongated and narrow channel of streaming plasma (Fig. 39). Numerical simulations confirmed that the main cause of the Earthward plasma motion in the bubble is the difference $S_{0}-S_{B}$ in the entropies. In particular, the Earthward motion is primarily controlled by such a depletion, whereas the initial speed of the bubble is a secondary factor (Birn et al., 2004). In the absence of a depletion, the kinetic energy of the fast flow will be dissipated rapidly by the compression/heating of plasma Earthward of the bubble. This explains why the depleted density and entropy inside the fast flowing plasma are observed in the BBFs, see, e.g., Fig. 2.

The final stage in the evolution of plasma bubbles is much less clear, as no detailed modeling results are available. It is anticipated that the internal cause of the inward motion will be exhausted after reaching the point where $S_{B}=S_{0}$, where the bubble becomes indistinguishable from the neighboring plasma tubes in terms of $p$ and $B$, and the accompanying processes may possibly include oscillatory motions around the braking point (Chen and Wolf, 1999). Diffusion-like 


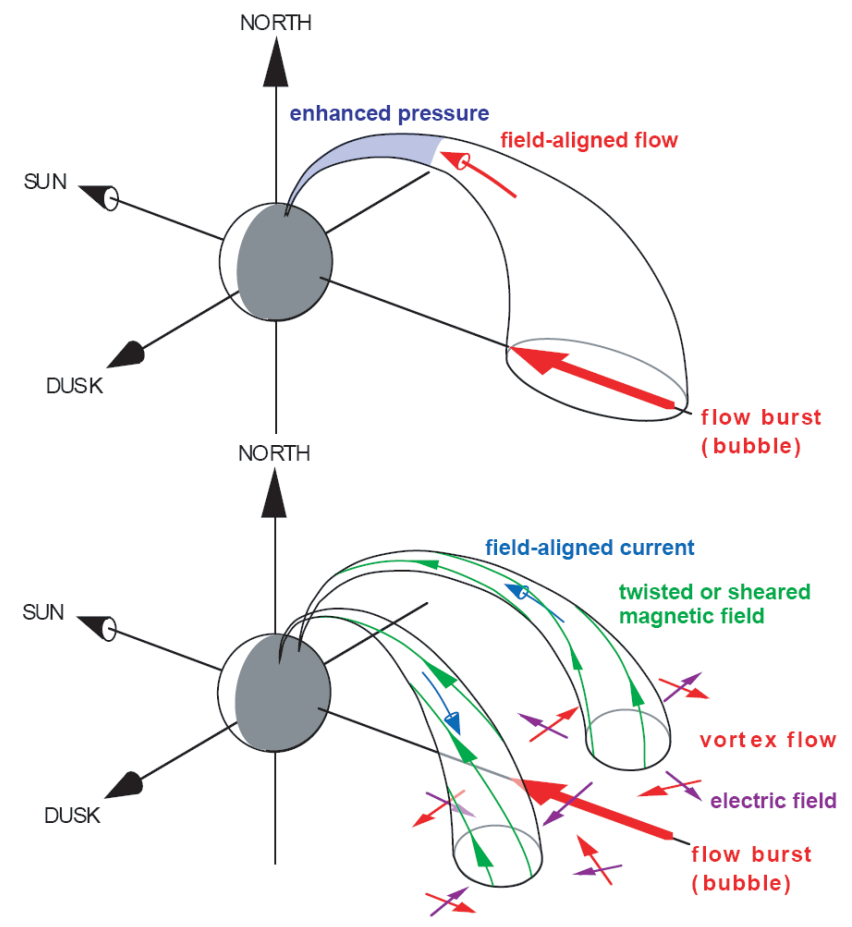

Fig. 39. Schematic of a plasma bubble. The upper panel emphasizes the plasma flows inside the bubble (including the field-aligned flow bringing to the temporary pressure increase in the bubble horn). Lower panel illustrates the distribution of flows (red arrows), E-field (magenta) and magnetic shear corresponding to the field-aligned currents at the bubble flanks. (From Birn et al., 2004).

processes (e.g., magnetic drifts) may contribute in smearing out the difference between $S_{B}$ and $S_{0}$, but their efficiency has not been investigated yet. An important detail is that sharp plasma gradients arising at the bubble front may also cause interchange and/or ballooning instabilities, which may make the bubble structured at different scales (TanDokoro and Fujimoto, 2005) and initiate/create the strong turbulence which is a well-known feature of the current disruption region.

The bubble is essentially a complicated 3-D structure, as shown in Fig. 39. The flow shear between the bubble and surrounding media create the magnetic shear (field-aligned current) which has a R1 sense (Figs. 39, 40). The similarity between the plasma bubble current system and the substorm current wedge is striking. The upward (downward) current is at the dusk (dawn) flank, and the total FAC reaches a few tenths of MA in the simulations (Birn et al., 2004). These simulations also showed the importance of plasma dynamics along the flux tube, with significant plasma compression occurring in the horns of the plasma bubble.

It is generally anticipated that main mechanism producing the bubbles could be magnetic reconnection, which reconnects flux tubes of lower density (taken from outer PS or the lobe) and provides the localized (in $X$ ) underpopulated closed plasma tubes on its Earthward side (see e.g., re-
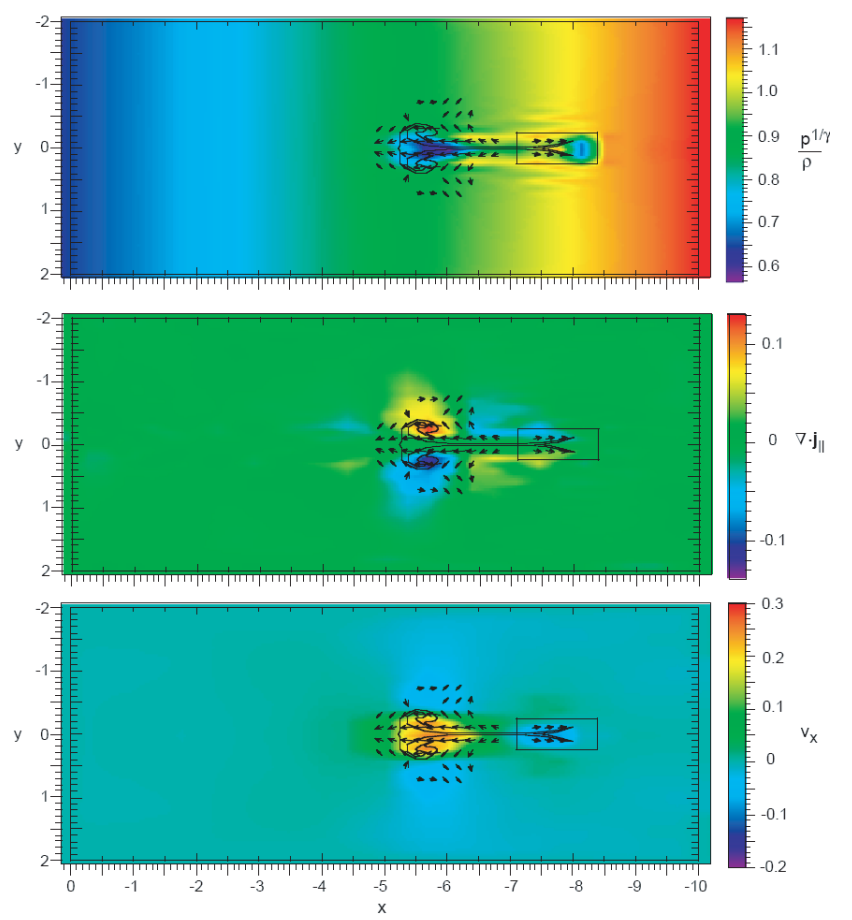

Fig. 40. Bubble properties in the equatorial plane at time $t=15$ (in normalized units) after its formation, including (from top to bottom): the entropy distribution, diversion of the parallel current density integrated over Z, and flow speed. (From Birn et al., 2004).

sults of numerical simulation in Fig. 32, and Birn and Hesse, 1996). The interchange motion of this reconnected, underpopulated tube then brings plasma inward into the closed flux tube region, e.g., into the ring current region. Although the above scenario includes interchange dynamics as a secondary process (in the sense of time sequence, but not of its importance), one should not exclude the possibility that interchange instability can sometimes form the bubbles. For example, in the plasma sheet regions with azimuthal pressure gradients which are required to support the large-scale fieldaligned currents (Golovchanskaya and Maltsev, 2003), the instability growth, direction of flow and propagation can all be changed as compared to the general case described above, making the interchange process easier to grow.

The origin of the small cross-tail scale of BBFs $\left(2-4 R_{E}\right.$, Sect. 2.1) is not yet completely understood. Some simulation studies suggest that narrow flow channels can be generated without explicitly prescribing the finite cross-tail extent of the reconnection region. Based on 3-D hybrid simulations, Shay et al. (2003) found that reconnection jets having a dawn-dusk width consistent with observations can be created spontaneously from random fluctuations present in the plasma sheet. However, the grid-cell size in the dawndusk $(y)$ direction used in their simulations was significantly larger than ion inertial length, so a concern remains that these results may not be numerically correct, i.e., the applicability 


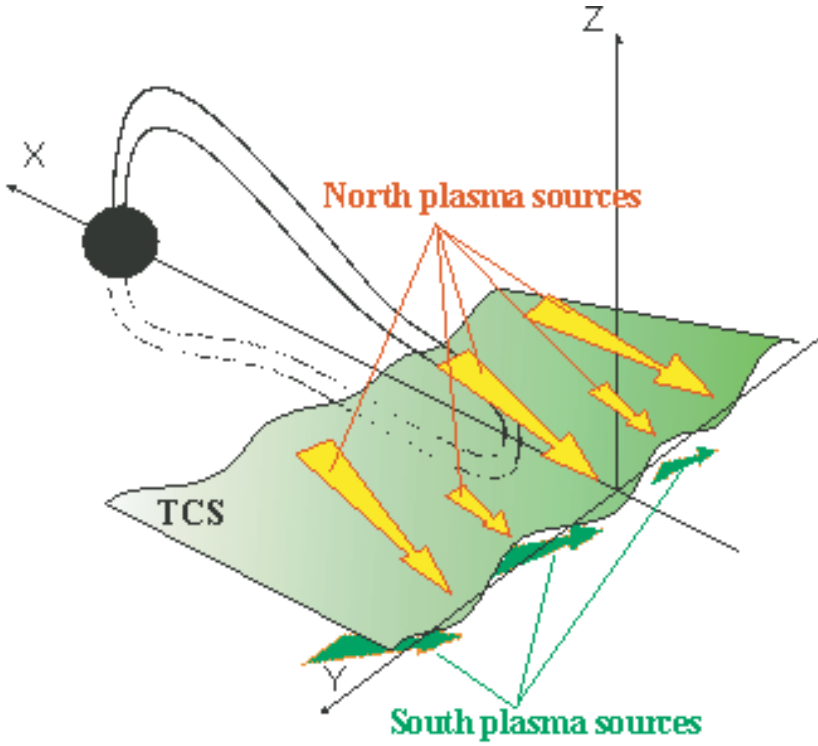

Fig. 41. Schematic of a mechanism of kink-like bending of the current sheet plane due to asymmetry of plasma sources in lobes.

of their result to the actual observations is questionable. On the other hand, Nakamura et al. (2002) show, based on 3-D hybrid simulations with a uniform anomalous resistivity in the $y$ direction, that the interface between the reconnection jet and pre-existing plasma sheet, both with a 2-D shape at the initial stage, is subject to interchange instability. Moreover, the instability creates magnetic bubble structures having a cross-tail width of a few $R_{E}$, reminiscent of bursty bulk flows. These results are significant in the sense that narrow flow channels can be produced without invoking a spatial localization in the cross-tail direction for the reconnection process.

\subsection{Current sheet flapping models}

The interpretations of the fast flapping events described in Sect. 2.7 may vary depending on the magnetic field geometry, the local plasma sheet conditions, as well as the magnetospheric conditions. Some observational features, such as strong tilts and flankward propagation, are unexpected discoveries and more work is needed to further clarify them, both experimentally and theoretically. Some mechanisms relevant to this phenomenon are reviewed in this section.

The flankward propagation of many flapping transients and its general similarity with spatial structure of BBFs indicates that they may have an internal source, located in the near-midnight sector of the magnetotail. For the direct MHD-type sheet deformation occurring during magnetic reconnection, it was suggested that in sheared magnetic configurations (with nonzero $B_{y}$ in the neutral sheet) the interaction of reconnected flux tubes with the sheet is asymmetric and this causes the up-down sheet motions on the flanks of the localized (in $Y$ ) reconnection region (Semenov et al., 1994; Shirataka et al., 2006) (see Sect. 3.2). However a direct relationship between the observed flappings and active processes in the plasma sheet, such as reconnection and flow bursts, is still unclear.

A number of wave generation mechanisms have also been discussed, including ion-ion kink (e.g., Karimabadi et al., 2003) or Kelvin-Helmholtz (e.g., Nakagawa and Nishida, 1989) instabilities, or a standing mode in the (neutral) current sheet (Fruit et al., 2002a,b). However, neither KelvinHelmholtz nor ion-ion kink waves propagate in opposite $( \pm Y)$ directions from the midnight region. The investigation of MHD eigenmodes of the Harris-type current sheet without a normal component (e.g., Lee et al., 1988; Louarn et al., 2004) also did not provide a solution, at least for the majority of observations.

PIC simulations of the evolution of non-Harris current sheets reproduces some elements of flapping, in particular the sheet surface corrugation with electric current rotation in the $Y Z$-plane (Sitnov et al., 2004, 2005). The current sheet thickness also temporally changes during flapping due to a combination of kink and sausage modes (Sitnov et al., 2005).

The ballooning-like mode in the high- $\beta$ plasma sheet with a large normal component of magnetic field was claimed to be able to propagate azimuthally in the flankward directions (Golovchanskaya and Maltsev, 2004). This model currently looks most suitable for explaining quasi-periodic, slip-type flapping observed in a quiet, thick plasma sheet.

Zelenyi et al. (2004); Malova et al. (2007) assumed that kink-type shapes of the current sheet can be driven by natural north-south asymmetry of the plasma sheet, and modified a self-consistent 1-D model of a thin forced current sheet to include asymmetric plasma sources. This asymmetry of plasma sources does not strongly influence the cross-tail current supported by meandering parts of ion orbits. However, the negative diamagnetic currents become much stronger at the source side due to the increase in plasma density in this region. To balance the pressure difference arising from this difference in the density, the current sheet shifts away from the source, relative to symmetric case.

This phenomenon might explain both dynamical oscillations of the position of current sheet and possibly radiallyelongated current sheet warping. In reality, northern and southern plasma sources might fluctuate corresponding to the variability of the mantle, plasma sheet, ionospheric sources, and/or solar wind. When the characteristic time scale of the temporal fluctuations is larger than the characteristic scale of the relaxation process, the entire thin current sheet will move up and down in $Z$ direction. Also sources with a finite scale along y direction may drive modifications of the current sheet, which are different in different local time sectors (Fig. 41). 


\subsection{Linear MHD response of the plasmasheet}

The plasma sheet fluctuations with periods ranging from seconds to minutes could correspond to: (1) MHD oscillations (Seboldt, 1990; Smith et al., 1997), (2) tearing modes (Pellat et al., 1991; Pritchett, 2001; Sitnov et al., 1998, 2000), and (3) various micro- or meso-scale instabilities such as: drift (Huba et al., 1980), cross-field (Lui, 1996), ballooning (Roux et al., 1991), and kinetic kink instability (Lapenta and Brackbill, 1997; Daughton, 2002). However, these waves do not have equally important energetic and structural consequences on the equilibrium of the sheet. For example, 1-D Harris sheets are stable to ideal MHD perturbations (Dahlburg et al., 1992). This, however, does not mean that MHD eigenmodes are negligible. The plasmasheet is an open system and, a priori, any external perturbations may trigger MHD eigenmodes with amplitudes that are directly linked to one of the initial perturbations. Fluctuations of the plasmasheet thus need to be considered as a mixture of stable modes and waves resulting from intrinsic instabilities. It is crucial to compare the criteria for their identification as well as quantitative models for interpreting their amplitudes. Such a model, based on a theory of the MHD linear response of 1D Harris sheet, is described below.

Although linear theories are unable to specify the amplitude of unstable waves, they can predict the amplitude of stable eigenmodes excited by known external perturbations. Thus assuming the Green function of the system and considering an external perturbation, the actual amplitudes of the excited eigenmodes are calculated. In the case of 1-D Harris sheet, this method has been developed by Fruit et al. (2002a,b) and applied to the interpretation of Cluster observations (Louarn et al., 2004; Fruit et al., 2004).

The method takes into account the inhomogeneity of the sheet, and the differential equation that describes the evolution of perturbations along the direction of inhomogeneity must be solved in the real space (Seboldt, 1990). After timeLaplace and space-Fourier transforms along the directions of homogeneity ( $x$ and $y$ ), the equation for the MHD perturbations is written in terms of the transverse displacement $\xi$ of the plasma (along $z$ ):

$\frac{d}{d z}\left[f_{\omega, k}(z) \frac{d \xi}{d z}\right]+g_{\omega, k}(z) \xi=S_{\omega, k}(z)$

where $f$ and $g$ are functions of $\omega, k_{x}, k_{y}$ and $z$. In the case of pure parallel propagation along $x\left(k_{y}=0\right)$ to which the following development is restricted, these coefficients become

$$
\begin{aligned}
& f_{\omega, k}=\rho_{e q}\left(v_{s}^{2}+v_{A}^{2}\right) \frac{\omega^{2}-k^{2} v_{c}^{2}}{\omega^{2}-k^{2} v_{s}^{2}}, \\
& g_{\omega, k}=\rho_{e q}\left(\omega^{2}-k^{2} v_{A}^{2}\right),
\end{aligned}
$$

where the different quantities $v_{S}, v_{A}$ and $v_{c}$ are the sound, Alfvén and cusp speeds, respectively. The cusp speed is given by

$v_{c}^{2}=\frac{v_{A}^{2} v_{s}^{2}}{v_{A}^{2}+v_{s}^{2}}$.

Equation (8) is a Sturm-Liouville equation. It can be shown that in some regions of the $(\boldsymbol{k}, \omega)$ space, when $f_{\omega, k}(z)$ does not vanish, it has a discrete spectrum of eigenvalues. The corresponding eigenfunctions form a complete orthogonal basis that can be used for expanding the Green function of the system. The second term of Eq. (8) is the source term related to the excitation. This equation was solved with boundary conditions corresponding to solutions embedded in the sheet, with the transverse displacement vanishing at infinity, and yields $\omega_{n}$ and $\psi_{n}(z)$, the eigenfrequencies and eigenfunctions, respectively. The Green function, which corresponds to the response to a unit source at a point $z^{\prime}$ and oscillating at the given $\omega$ and $\boldsymbol{k}$, can be constructed from the discrete set of eigenfunctions (Eq. (19) in Fruit et al., 2002a):

$G_{\omega, k}\left(z \mid z^{\prime}\right)=\sum_{n=0}^{+\infty} \frac{\psi_{n}(z) \psi_{n}\left(z^{\prime}\right)}{\omega^{2}-\omega_{n}^{2}}$.

As usual, the eigenfrequencies $\omega_{n}$ are the poles for the Green function considered as a function of the complex variable $\omega$. Hence the solution of Eq. (8) with a given source term becomes

$\hat{\xi}_{z}(\omega, k, z)=\int_{-\infty}^{+\infty} d z^{\prime} G_{\omega, k}\left(z \mid z^{\prime}\right) S_{\omega, k}\left(z^{\prime}\right)$

The reconstruction of the complete spatial/temporal fluctuation associated with the given source is then made by inverting the Laplace and Fourier transforms. The final result can be formally written as:

$$
\begin{array}{r}
\xi_{z}(t, x, z)=-\frac{i}{2 \pi} \sum_{n=0}^{\infty} \int_{-\infty}^{+\infty} d k e^{i k x} \\
\times \frac{\psi_{n}(z) \psi_{n}\left(z^{\prime}\right)}{2 \omega_{n}} \\
\times\left[S_{\omega_{n}, k} e^{-i \omega_{n} t}-S_{-\omega_{n}, k} e^{i \omega_{n} t}\right]
\end{array}
$$

Once the transverse displacement is computed, the magnetic field and the thermal pressure perturbations can be obtained. To compare the observations, shown in Fig. 22 with the MHD linear response model, Eq. (8) was solved with boundary conditions corresponding to evanescent modes in the lobes and an initial vertical displacement. The excited eigenmodes are then calculated. The corresponding fluctuations are shown in Fig. 42.

The colored isocontours of the thermal pressure (in $\mathrm{nPa}$ ) are displayed versus time together with three black lines corresponding to the iso-magnetic field, the central line being the neutral sheet. From this figure, one gets a picture of the 


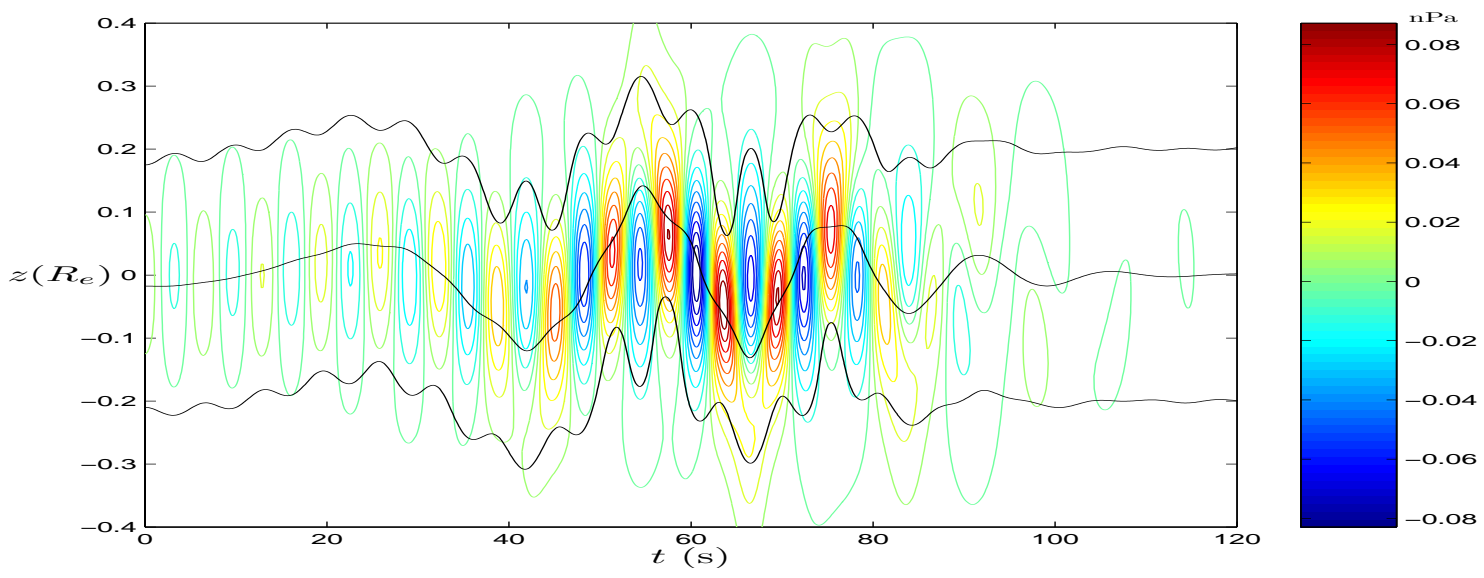

Fig. 42. Temporal evolution of the current sheet responding to an initial displacement located at $50 a=10 R_{e}$. The three black lines correspond to a constant $B_{x}$ component and the colored isocontours represent the thermal pressure fluctuations. (From Fruit et al., 2004).

temporal evolution of the plasma sheet at a fixed $x$ position. This plot is equivalent to the one on the lowermost panel of Fig. 22.

The purpose of the analysis was not to achieve an exact reproduction of the sheet configuration described in Fig. 22, but to compare the main characteristics of the oscillations with the observed ones. The theoretical response of the plasma sheet exhibits both kink and sausage oscillations. The kink mode has a period of $\sim 20 \mathrm{~s}$ and an amplitude of about $0.3 R_{E}$, which is in a very good agreement with the observations. The sausage oscillations affect the thickness of the sheet. They have period of the order of $6 \mathrm{~s}$ or a frequency of $\sim 0.16 \mathrm{~Hz}$, which is also close to the second peak at $0.13-$ $0.14 \mathrm{~Hz}$ observed in the experimental data. Furthermore, the ratio between the amplitude of the fundamental kink and the sausage mode (around 5) is close to the observed one.

The agreement between the observed frequencies and amplitudes of the peaks and the ones predicted by the model is remarkable. It was not obvious that an initial excitation characterized by a broad spectrum could generate well-defined sinusoidal oscillations in the frequency ranges actually observed. This shows that the Harris sheet behaves like a frequency filter, each mode being excited at specific frequencies. This model could thus give a relatively simple interpretation of some of the observed large compressional oscillations of the plasma sheet.

\subsection{Particle dynamics in reconnection region}

In general magnetic reconnection is associated with parallel electric field (Schindler et al., 1988; Sonnerup, 1988) and this has been demonstrated in the case of the magnetopause using global MHD simulations (Siscoe et al., 2001). The main mechanism is the meandering Speiser motion, through which the charged particles can gain energy from the dawn-dusk reconnection electric field during their gyro-motion under the reconnecting magnetic field (Speiser, 1965; Sonnerup,
1979). The time dependent fields of MHD simulations has been used to study the acceleration of test particles (Birn, 2007). The localized electric fields due to dipolarization has been found to energize both ions (non-adiabatic) and electrons (adiabatic) and can cause energetic particle injections into the near tail and inner magnetosphere. In the following other but related mechanisms of particle acceleration are discussed, focusing on processes directly connected to the reconnection and relevant also to mid- and far-tail observations.

In a simple model suggested by Lyons and Speiser (1982) the gain in particle energy can be easily calculated in terms of Fermi-type acceleration due to the reflections from the moving "mirror" which is the corresponding de Hoffman-Teller frame $V_{H T}=c E_{y} / B_{n}(x)$, where $E_{y}$ is the dawn-dusk electric field penetrating into the magnetotail and $B_{n}(x)$ is the local value of the transverse component of the magnetic field in the vicinity of the CS (i.e. magnetic reversal) plane. Finally, the energy gain of the non-adiabatic particles could be evaluated as

$\Delta W_{i}(x)=\frac{m_{i}\left(2 \cdot V_{C}(x)\right)^{2}}{2}=2 m_{i}\left(\frac{E_{y}}{B_{z}(x)}\right)^{2}$

Typically $\Delta W_{i}$ is of the order of few $\mathrm{keV}$, and initial ion energy $\sim$ few hundred $\mathrm{eV}$ could be neglected in these estimates.

As discussed in Sect. 2.10, the energetic, non-thermal electrons with $\mathrm{MeV}$ energies are known to commonly exist in the magnetotail, while the thermal plasma temperature is of the order of $\mathrm{keV}$. Therefore the typical thermal electron gyro-radius is quite small compared to the characteristic scale length of the plasma sheet, and the electron motion is basically adiabatic except for the magnetic diffusion region and the slow shock boundary between the lobe and the plasma sheet. The non-adiabatic motion is of major importance in non-thermal particle acceleration.

Several possible mechanisms may contribute to the nonadiabatic motion of particles. The particle scattering by 
turbulence is recognized as one of the essential processes for the break-down of adiabaticity of particle dynamics, and forms the basis of efficient energy transfer from waves to particles. During plasma sheet crossings by satellites, largeamplitude MHD turbulence and whistler bursts are often observed both in the plasma sheet and in the boundary layer (e.g., Gurnett et al., 1976), and the turbulence may play an important role in both plasma thermalization and energetic particle production (e.g., Kennel, 1995; Hultqvist et al., 1999).

Recently a comparative study of satellite observations and numerical simulations revealed that the small-scale fluctuations responsible for the non-adiabatic electron motion can be excited in the reconnection region. It is known that a strong Hall electric current is induced in the reconnection region when the plasma sheet thickness becomes as thin as the ion inertia length (see Sect. 3.5). The electron distribution function in the boundary layer between the lobe and the plasma sheet consists of both the cold electrons flowing into the X-type region and the accelerated electrons flowing out from the diffusion region. This bump-on tail distribution can excite a large-amplitude electrostatic wave (e.g., Hoshino et al., 2000), which is related to the observation of solitary electrostatic waves in the magnetotail (Cattell et al., 1994; Kojima et al., 1994; Cattell et al., 2005). In the plasma sheet, the velocity difference between ions and electrons may be regarded as the free energy source for wave generation as well (e.g., Coroniti, 1985; Drake et al., 2003). Such strong plasma thermalization may modify the structure in and around the diffusion region, which in turn affects the Hall electric current system and leads to the dynamic plasma sheet with transient plasma behavior. The scale of the electrostatic field found in the plasma sheet is of the order of several tens of Debye length, but the magnitude of the electric field is much larger than the reconnection electric field, i.e., the dawndusk electric field. Therefore, the micro-scale processes appear to control the global dynamics. This kind of multiscale coupling process is beginning to shed light not only on the plasma dynamics but also on the non-thermal particle acceleration.

Another class of non-adiabatic process occurs when the gyro-radius of the particle becomes comparable to the characteristic scale length of the magnetic field curvature (Büchner and Zelenyi, 1989; Delcourt et al., 1996). During magnetic reconnection, the curvature of the reconnecting magnetic field line, $l_{\text {cur }}$, becomes as small as the thickness of the plasma sheet, i.e., $l_{\mathrm{cur}} \sim B_{n} / B_{0} \lambda$, where $B_{0}$ and $B_{n}$ are the magnetic field in the lobe and the neutral sheet, and $\lambda$ is the plasma sheet thickness. Therefore, the ratio of the reconnecting magnetic field curvature to the gyroradius at the neutral sheet, $\kappa^{2}$, can be expressed by,

$\kappa^{2}=\left(\frac{B_{n}}{B_{0}}\right)^{2} \frac{\lambda}{r_{g}}$,
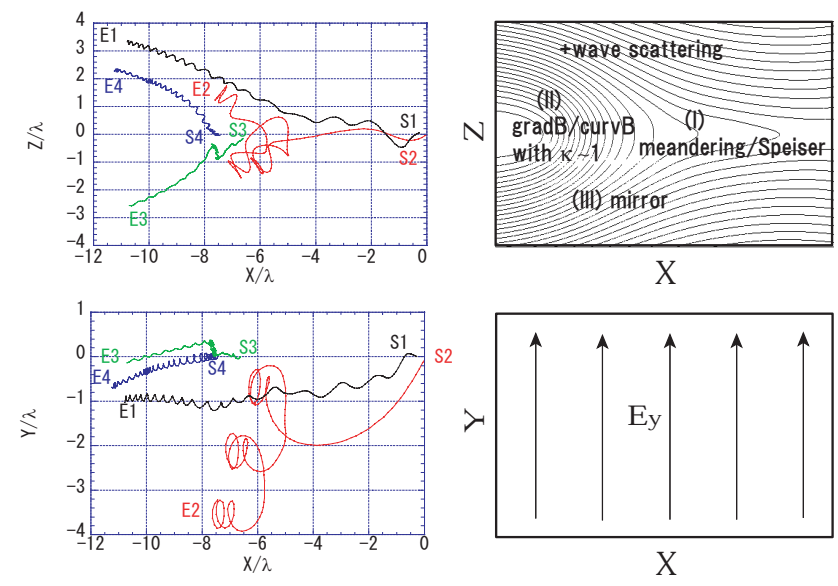

Fig. 43. Several electron trajectories in the reconnection region. (From Hoshino et al., 2001).

where $r_{g}$ is the gyro-radius defined by the lobe magnetic field $B_{0}$. It is recognized that most of thermal ions may enter the regime where their motion becomes chaotic, but this is not the case for electrons. However, suprathermal electrons may also show chaotic motion near the diffusion region, where the amplitude of the reconnecting magnetic field is small.

Most of the above electron acceleration processes have been demonstrated by the particle-in-cell (PIC) simulations (Drake et al., 2003, 2006), and non-thermal energy spectra for electrons have been obtained in the reconnection region. Figure 43 shows several typical electron trajectories around the reconnection region obtained by PIC simulation (Hoshino et al., 2001). The top left panel shows the electron orbit in the $x-z$ plane, with the reconnection magnetic field lines included, while the bottom left panel shows the orbit in the $x-y$ plane, where the convection electric field is induced. As a reference, the top right and bottom right panels show cartoons of the reconnecting magnetic field lines in the $x-z$ plane and the electric field $E_{y}$ in the $x-y$ plane, respectively. The particle denoted by S1-E1 is the most typical Speiser orbit that contributes to the PSBL electron beams. An electron is transported from the X-type region to the lobe boundary region almost along the magnetic field line. The particle can gain energy in the magnetic diffusion region near $-6<X / \lambda<0$, where $\lambda$ is the thickness of the plasma sheet, which is comparable with the ion inertia scale length. The trajectory with S2-E2 is a kind of chaotic orbit with $\kappa \sim 1$, and the energy gain is large, because the particle travels a large distance in the negative $y$ direction. The particle with $\kappa \sim 1$ can be scattered toward the large pitch angle region because of a centrifugal force perturbing the particle gyromotion in weak magnetic field regions. Such particles tend to stay in the plasma sheet for a longer time, which in turn contributes to their preferential acceleration during gradient/curvature drift motion. The trajectories of S3-E3 and S4-E4 are examples of cross-field diffusion and pitch-angle 


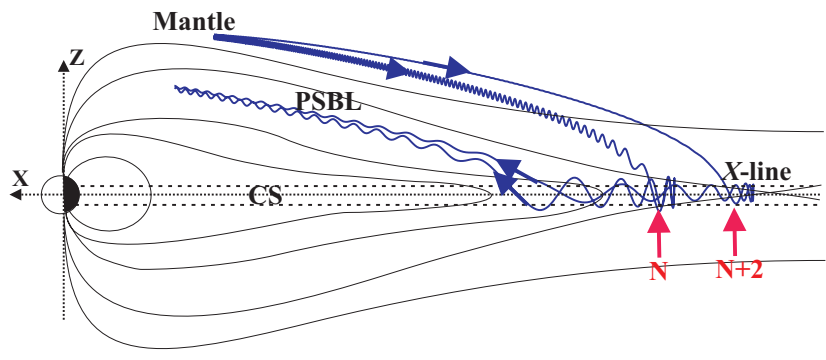

Fig. 44. Projection of typical trajectories of particles onto the $(X, Z)$ plane as they move towards the CS from the source in the mantle, then interact with the CS at different resonant sites (having numbers $N, N+2$ ) and are ejected from the CS forming field-aligned beamlets moving Earthward. These beamlets intersect at some $X$ location because of combining action of "place of birth" and velocity filter effects. The simulation of particle trajectories was performed in the 2-D Birn-Zwingmann equilibrium analytical magnetic field model using the constant uniform dawn-dusk electric field $E=0.1 \mathrm{mV} / \mathrm{m}$.

scattering, probably due to wave-particle interactions with the self-generated turbulent fields in the reconnection region. Note also that intense non-thermal energetic electrons have been observed by Cluster (Imada et al., 2007) in the magnetic field pile-up region where the reconnection jet collides with the pre-existing plasma sheet plasma at rest.

In addition to the above acceleration, large-amplitude polarization electrostatic waves can play an important role in electron energization as well. Recent Cluster studies reported that the amplitude of electric fields observed at the lobe/plasma sheet boundary are as large as $100 \mathrm{mV} / \mathrm{m}$ (Wygant et al., 2005). In association with the Hall electric current, a north-south polarization electric field perpendicular to the boundary can be generated. Hoshino (2005) argued that the polarization electric field induced at the boundary can contribute to the generation of suprathermal electrons, together with the non-adiabatic processes discussed above. Some electrons can be trapped by the polarization electric field, and during the trapping phase can gain energy from the reconnection electric fields through the so-called surfing acceleration (Sagdeev and Shapiro, 1973; Katsouleas and Dawson, 1983).

\subsection{Non-adiabatic ion acceleration in the current sheet}

In the vicinity of a magnetic X-line in the magnetotail current sheet, charged particles originating from the mantle and/or ionosphere experience strong non-adiabatic acceleration (Lyons and Speiser, 1982), and increases in their initial velocities by up to ten times (see Eq. 15). As a result of such non-adiabatic processes, fast ion beams (beamlets) moving along magnetic field lines are formed in the interface region between open lobe magnetic field lines and closed field lines, containing hot isotropic plasma of the plasma sheet (AshourAbdalla et al., 1993). It has been noted (Lui et al., 1977;
DeCoster and Frank, 1979) that these high-velocity ion structures are mostly confined to a $1-2 R_{E}$ layer near the boundary of the plasma sheet. This region, which is named the Plasma Sheet Boundary Layer (PSBL) (Eastman et al., 1984) has been studied actively.

The global PSBL structure is most clearly observed in the high-latitude auroral region where it is manifested as VDIS (Sect. 2.9, and Fig. 24 Zelenyi et al., 1990; Sauvaud et al., 1999; Keiling et al., 2004; Sauvaud and Kovrazhkin, 2004). In Sect. 2.9 various mechanisms leading to the ion energy dispersion in the auroral region were discussed, viz. both velocity filter and "place of birth" effects are responsible for the final dispersion acquired by particles precipitating to the auroral ionosphere.

Additional physical insight came after the discovery of socalled current sheet resonances (Burkhart and Chen, 1991; Ashour-Abdalla et al., 1993). It appears that particles interacting with the current sheet at a certain location $X_{0}$ could acquire an energy $\Delta W\left(X_{0}\right)$, but, as a rule, in a form of "thermal" energy. While this could explain the energization of plasma sheet particles, but to produce collimated, well-organized PSBL ion beams additional "resonant" conditions need to be met at the acceleration site. The physics behind these "resonance conditions" is relatively straightforward, viz. the appearance of regular islands in the generally stochastic particle dynamics in the current sheet.

At certain resonant locations, stochastic effects, which mostly influence particles during their entry to and exit from the current sheet region (see Fig. 44), these effects counteract each other and the particle behaves as if its motion is regular and gains its kinetic energy mostly in a field-aligned direction. Figure 44 presents particle trajectories simulated in the 2-D Birn-Zwingmann equilibrium analytical magnetic field (Zwingmann, 1983) using a constant uniform dawndusk electric field $(E=0.1 \mathrm{mV} / \mathrm{m})$. The behavior of ions is characterized by the parameter of adiabaticity $\kappa$ (Eq. 16). In the vicinity of the neutral plane the magnetic field can be considered to have a parabolic profile with the normal component $B_{z}$ depending on the distance from the Earth, $B_{z}=B_{z}(X)$.

For such simple models $\kappa$ can be expressed in the form

$\kappa=\left(\frac{e^{2}}{2 m_{i}}\right)^{1 / 4} B_{n} B_{0}^{-1 / 2} L^{1 / 2} \Delta W^{-1 / 4}$

Combining this expression with Eq. (15) for Speiser acceleration, one obtains

$\kappa(x)=\delta \cdot B_{n}(x)^{3 / 2}$,

where

$\delta=\sqrt{\frac{e}{m} \frac{L}{B_{0} E_{y}}}$.

A detailed analysis of the resonance conditions (AshourAbdalla et al., 1993; Zelenyi et al., 2007) shows that to 
minimize chaotic scattering and to produce energetic fieldaligned ion beams the local value of $\kappa(x)$ should satisfy the condition

$\kappa(x)_{R}^{N} \simeq C_{1} / N$,

where $N$ has an integer value and $C_{1}$ is a constant $\left(C_{1}=0.763\right)$. Combining Eqs. (15) (16) (17) yields the scaling for particle energies accelerated at the different resonant sites:

$W\left(X_{R}^{N}\right)=2 m_{i}\left(\frac{C_{1}}{\delta}\right)^{-4 / 3} E_{Y}^{2} N^{4 / 3}=C_{0} N^{4 / 3}$

This nonadiabatic resonant acceleration model therefore predicts the appearance of a sequence of resonant sites $X_{R}{ }^{1}$, $X_{R}{ }^{2}, X_{R}{ }^{3}, X_{R}{ }^{4} \ldots$, where localized, highly-accelerated and collimated ion beams with the energies $W\left(X_{R}{ }^{N}\right)$ are produced (see Fig. 44, where only two resonances $N$ and $N+2$ are indicated). Typical trajectories of the beamlet particles accelerated at even $(N=2,4,6, \ldots)$ and odd $(N=3,5,7, \ldots)$ are shown in Fig. 45 for cases $N=8$ (where the particle exits from the hemisphere opposite to the one into which it entered) and $N=15$ (where the particle exits from the the same hemisphere into which it previously entered).

The dispersion of each beam accelerated at particular resonance (referred to as a beamlet) is determined by a combination of the velocity filter effect and "place of birth" effect, acting in opposite ways. Their simultaneous operation may bring together at some location $(X, Z)$ beamlets accelerated at different resonances and having different energies $\left(V_{\|}\right)$(see Fig. 44 which shows the intersection of trajectories of beamlets accelerated at different resonances with numbers $N$ and $N+2$ ). Since at the current sheet resonant sites the energy-gain of ions almost corresponds to the kinetic energy of the field-aligned moving ions, a scaling law for the velocity of the subsequent resonant structure accelerated at the $N$-th resonance can be obtained as $V_{N}=C_{0}^{\prime} \cdot N^{2 / 3}$, where $C_{0}^{\prime}$ is a normalization factor.

Note that in the case of symmetric sources of accelerated particles in the northern and southern mantles, the beamlet observed near the Earth normally consists of ions coming from the different hemispheres and accelerated in neighboring resonances. However, in the general case of asymmetric (mantle) sources of particles populating the plasma sheet, the intensity of beamlets accelerated at neighboring $N$ and $N+1$ resonances might be different. Consider the case when the northern source dominates due to, for example, the seasonal effect. The particles coming from the Northern Hemisphere will be resonantly reflected, as almost coherent accelerated beams, to the Northern Hemisphere at, for example, odd resonances $(N=1,3,5, .$.$) and transmitted to the Southern Hemi-$ sphere at the even ones $(N=2,4,6, .$.$) . The differences in the$ particle trajectories in even and odd resonances are illustrated in Fig. 45. In the same way, particles originating from the southern mantle should be ejected to the opposite (Northern) hemisphere from even resonances but if the intensity of the
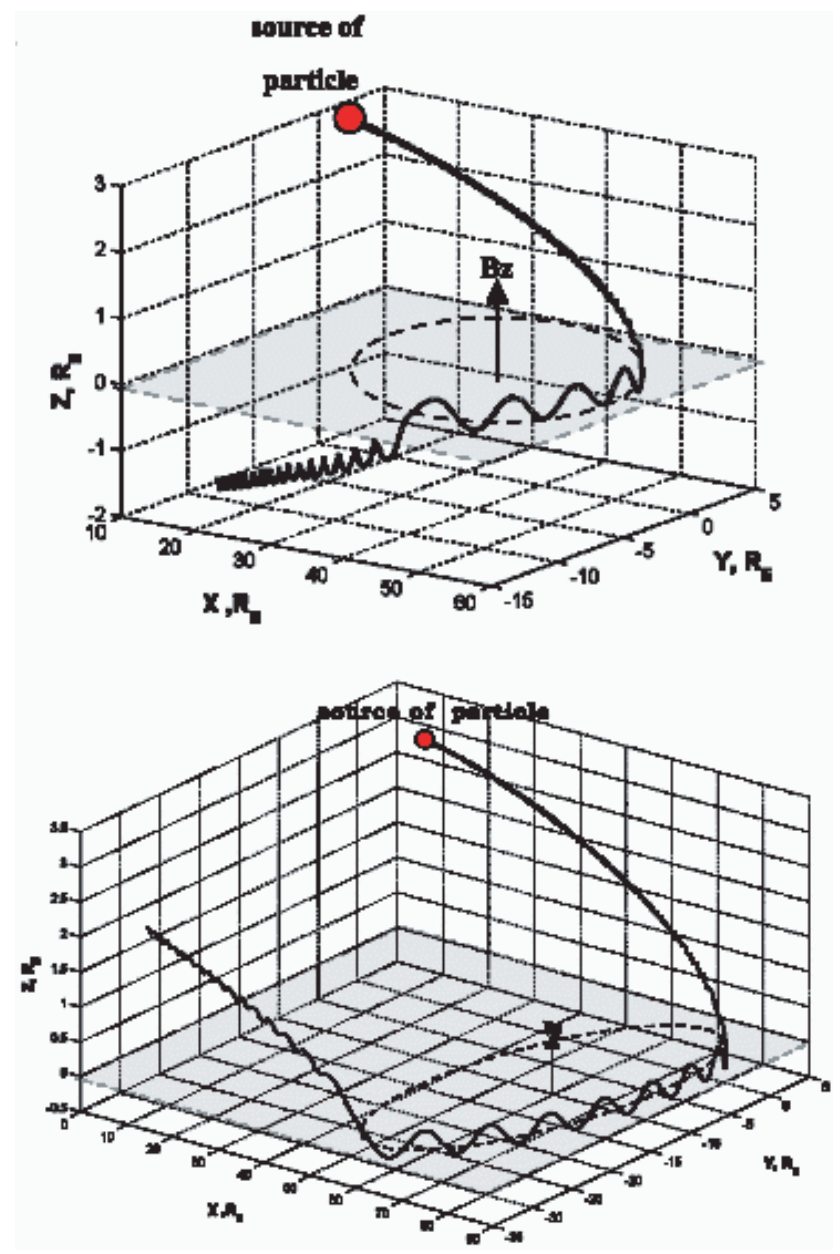

Fig. 45. Trajectories of non-adiabatic ions in the de Hoffman-Teller coordinate frame. The picture illustrates the combination of meandering motion and slow rotation in the $B_{z}$ field. Both trajectories shown correspond to the resonance condition when the chaotic scattering is almost absent. (a) even resonance $(N=8)$ : entry/exit are in opposite hemisphere; (b) odd resonance $(N=15)$ : entry/exit are in the same hemisphere.

southern source is weak, it is expected that the intensity of such beams will also be low and in the Northern Hemisphere, leading to weak or even an absence of beams accelerated at the even resonances. Thus in the PSBL of a particular hemisphere will yield a set of beamlets accelerated at $N$ and $N+2$ resonances.

Cluster observation of the beamlets (Sect. 2.9) was directly compared with the resonance theory by Malova et al. (2007). In order to compare the observations with the resonance scaling law predicted by the theory, the ratio, $V_{1} / V_{2}$, where $V_{1}$ is the ion parallel velocity corresponding to low-speed maximum of the double-peaked distribution function and $V_{2}$ is the parallel velocity corresponding to the high-speed maximum, is calculated from the observations and compared with the resonance level, $\left[\frac{N}{N+2}\right]^{2 / 3}$, where $N$ is the order of the 


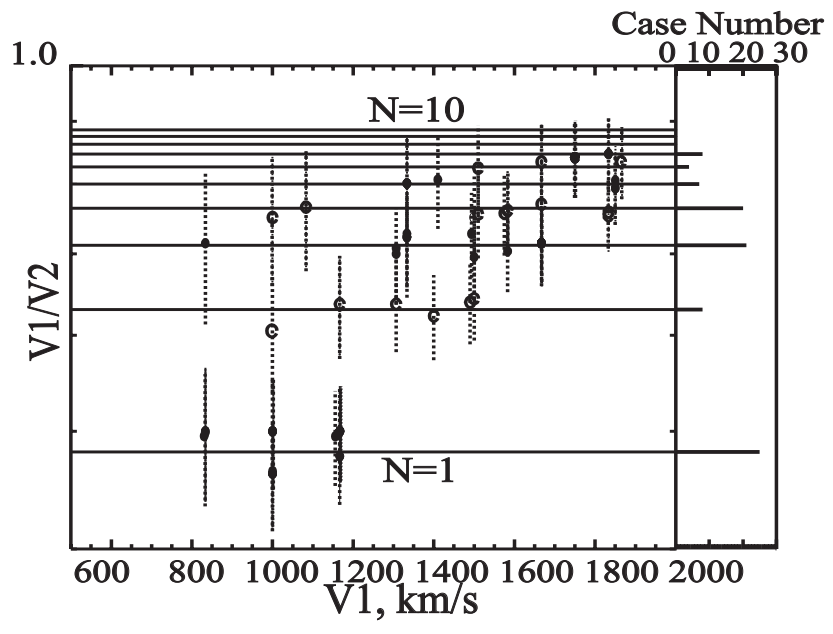

Fig. 46. Statistical verification of the scaling law for resonance structures predicted by Ashour-Abdalla et al. (1993). $V_{1} / V_{2}$ (empty and black circles) is plotted against $V_{1}$. The resonance level, $\left[\frac{N}{N+2}\right]^{2 / 3}$, for $N=1, . ., 10$ are shown by horizontal lines. The solid bars shown in the right part of the figure indicate the number of measurements of double-peaked distributions corresponding to the each particular resonance level. (From Zelenyi et al., 2006).

resonance. For the case of ion distributions consisting of two peaks, well separated in velocity space, an asymmetry of the mantle sources mentioned before was assumed. Data from 93 measurements of double-peaked distribution functions, which looked similar to that presented in Fig. 25, were analyzed.

Figure 46 shows the distribution of $V_{1} / V_{2}$ versus $V_{1}$. The levels of $\left[\frac{N}{N+2}\right]^{2 / 3}$ for different $\mathrm{N}(N=1, . ., 10)$ are shown by the horizontal lines. Vertical dotted lines indicate the error of $V_{1} / V_{2}$ caused by the finite width of each energy channel. On the right side of the figure, the number of cases with "doublepeaked" distributions used for the statistics for each resonance level are shown with solid bars. The ratio of $V_{1} / V_{2}$ are in good agreement with the resonant theory, independent of the velocity of the low-speed beamlet $\left(V_{1}\right)$. However, as $N$ increases the interval between the neighboring resonance lines shrinks and becomes less than the measurement error of the $V_{1} / V_{2}$ estimate. For $N \geq 3$ the comparison is already questionable. As presented above, for higher velocities (i.e. higher $N$ values) beamlets begin to get smeared and higher order beamlets become undistinguishable not only due to uncertainties of their detection, but also due to intrinsic overlapping of adjacent higher-order resonances.

In general, available magnetotail data conform with the predictions of the theory, although in the auroral region experimental evidence for beamlet scaling are not very abundant. One of the reasons for this is that for observations made near the Earth, where ion beams produced by different sources could overlap, resonance structures in the ion distributions are generally smeared out. The search for manifesta- tions of resonant effects in the PSBL region is very important for in-situ investigations of this phenomenon.

\subsection{Multiscale properties of the magnetosphere}

The mesoscale phenomena in the magnetosphere have been discussed in the previous sections based on their phenomenology and the physical mechanisms underlying the transient and localized processes. These processes constitute the multiscale phenomena of the magnetosphere consisting of micro-, meso- and macro- scale processes. Although a majority of the observed characteristics discussed in Sect. 2 were explained in the framework of MHD processes, kinetic processes in thin current sheets, for example, are also essential in the understanding of some of these observations. On the largest scale, the global features of the magnetosphere, such as plasmoid formation and release, correspond to a coherent response that affects the entire magnetosphere and arise from the strong coupling between its different components (Siscoe, 1991). The conspicuous global response of the magnetosphere, however, should be viewed as a component of the multiscale phenomena (Sharma et al., 2005b).

The magnetospheric multiscale phenomena has three main origins, viz. driving by the turbulent solar wind, nonlinearity, and cross-scale coupling among the plasma processes. Due to the wide range of space and time scales underlying the plasma processes in the magnetosphere, the multiscale phenomena are not understood well in terms of first principle models. This is particularly true of the coupling among the fluctuations with different scales, and has prompted different approaches to the understanding of their origins and consequences (Sharma et al., 2005a). In one approach the fluctuations are viewed as manifestations of turbulence (Borovsky et al., 1997; Borovsky and Funsten, 2003), with the objective of understanding transport processes and their role in magnetic reconnection and magnetic field structure in the magnetotail. These studies have addressed the nature of the turbulence, i.e., eddy or Alfvén wave turbulence, and the results favor eddy turbulence. The transient nature of the bursty bulk flows (BBF), discussed in Sect. 2.1 is akin to intermittency in turbulence, both in terms of the burstiness or variability in time, as well as the amount of energy associated with them. This has motivated studies of Cluster magnetic field observations using techniques based on multi-fractality (Vörös et al., 2003). The properties of the local intermittence measure (LIM) computed from the $B_{x}$ component of the magnetic field from Cluster $\mathrm{C} 3$ spacecraft show significant variation during the periods with distinct characteristics, for example, during the period shown in Fig. 47, the current sheet had a bifurcated structure and was subject to flapping between 10:55 and 11:05 UT on 29 August 2001 (Runov et al., 2003b) and the LIM values for the small scale (red line) and large scale (blue line) variations differ significantly. This indicates weak cross-scale coupling or dominantly coherent features, as expected during a large-scale flapping of the current sheet. 


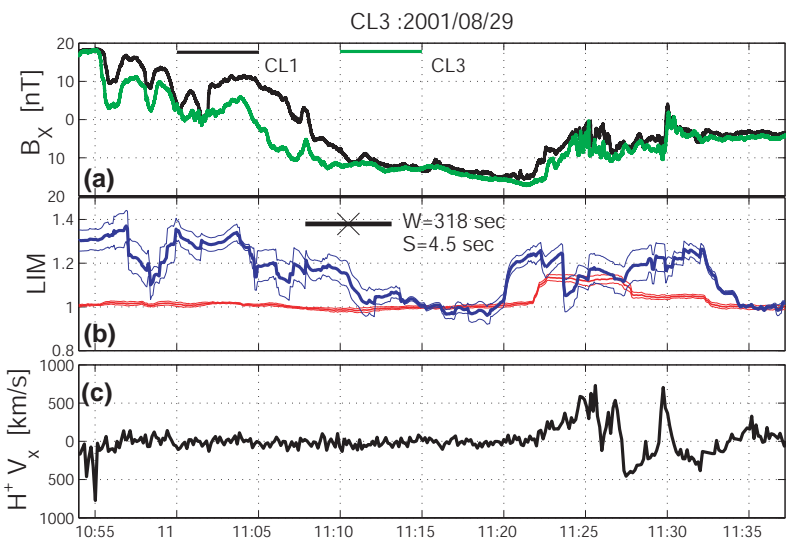

Fig. 47. The magnetic field component $B_{x}$ measured by Cluster 1 and 3 spacecraft on 29 August 2001 and the local intermittence measure (LIM) from C3. The current sheet was bifurcated in structure and was subject to flapping between 10:55 and 11:05 UT (Runov et al., 2003b). The $B_{x}$ components at $\mathrm{C} 1$ and $\mathrm{C} 3$ (panel a) have similar variations but at different levels. The LIM values for the small-scale (red line) and large-scale (blue) variations differ significantly during the current sheet flapping and bifurcation, indicating weak cross-scale coupling or dominantly coherent features. However, the two measures are close when $B_{x}$ is quiet or is accompanied by flow bursts, as shown by proton data in panel (c). The latter indicates turbulence, with the expected strong cross-scale coupling. (From Vörös et al., 2003).

However the two measures have similar values for the rest of the epoch, viz. when $B_{x}$ is quiet or is accompanied by flow bursts, as shown by proton data in panel (c). This again is expected as there is strong cross-scale coupling in turbulence. These results show that the fluctuations can not be characterized simply as manifestations of turbulence alone.

In another approach motivated by the need for a more comprehensive model, extensive observational data, especially long time series data from ground-based and spacecraftborne measurements, have been used to model the global and multiscale phenomena of the magnetosphere based on the recent developments in the theory of nonlinear dynamics and complexity (Sharma et al., 2005b). These models describe the dynamics, albeit in a reconstructed state space, and embody the features inherent in the data, independent of modeling assumptions. In this approach the dynamics of the magnetosphere is described in a state space constructed from the data, and the dominant or global features are obtained by averaging or coarse-graining over relevant scales, thus generating a mean-field type description (Ukhorskiy et al., 2004; Sharma et al., 2005b).

The multiscale features of the magnetosphere are often expressed in terms of power law distributions of the scale sizes. Such power law distributions have been obtained from the extensive data of auroral electrojet indices and solar wind variables. The power law distributions has led to views of the magnetosphere as an avalanching system (Uritsky et al.,

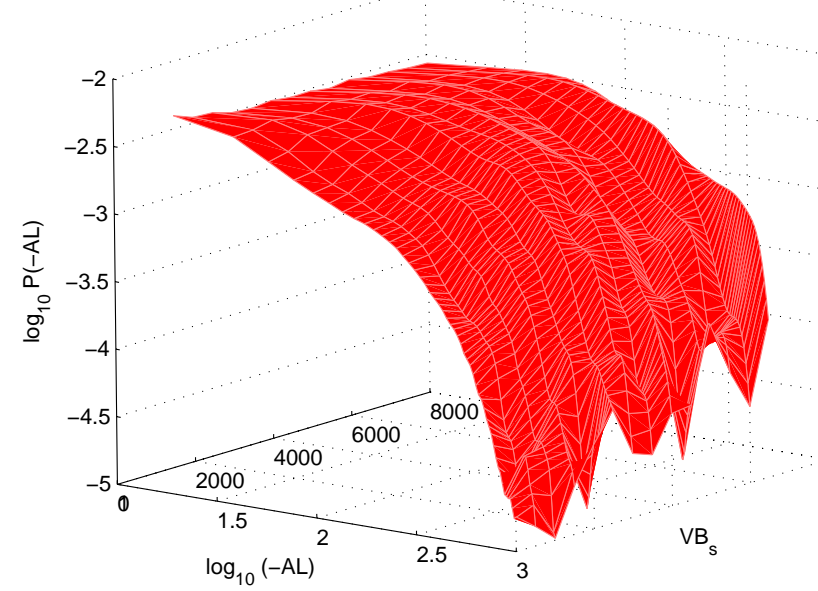

Fig. 48. Magnetospheric activity during substorms. The probability distribution function of AL index for different levels of solar wind induced electric field $V B_{z}$. The shape of the probability distribution function depends on the solar wind $V B_{z}$, and the deviation from the power law is stronger for stronger driving by the solar wind. The magnetospheric response does not exhibit scale-free response. (From Sharma et al., 2006).

2002). The burst life time distribution of the solar wind variables show scale-free behavior but the magnetosphere exhibits a significant deviation with preferred lifetimes comparable to substorm time scales, showing the co-existence of global and multiscale features (Freeman et al., 2000). The probability distribution functions (pdf) of magnetospheric response to different levels of solar wind driving provide a simple but important insight into the dynamic nature of the magnetosphere. Such pdfs are readily computed using the AL index as the magnetospheric response to the solar wind electric field $V B_{z}$, and are shown in Fig. 48. Studies of correlated data of the solar wind and the magnetosphere show a lack of scale-free behavior over the whole range of data and multiple scaling indices are needed to describe the entire spectrum (Hnat et al., 2003; Zaslavsky et al., 2007).

Many features of the magnetotail can be viewed within the framework of a general theory of open systems which are in non-equilibrium steady states (NESS). In such systems the steady state is attained only on the average and they exhibit substantial internal variability. The variability is not just small fluctuations on the top of an equilibrium but is an essential element in maintaining such quasi-equilibria. Such multiscale properties are inherent in the magnetosphere (Sharma et al., 2005b; Zelenyi and Milovanov, 2004) and the mesoscale processes discussed in this paper form a major part. 


\section{Discussion}

As shown in the previous sections, the classical 2-D Hones or Dungey type magnetotail pictures are not sufficient to explain some essential 3-D features of magnetotail dynamics. The long list of the mesoscale transients discussed in Sect. 2 indicates the complexity of the magnetotail processes, which has different roots and consequences. The most obvious reason for this complexity is that different processes (reconnection, instabilities such as interchange mode) can produce mesoscale structures in a number of ways. Reconnection itself can operate in an impulsive (Sect. 3.1), localized (Sect. 3.2), or mulitple (Sect. 3.3) regime, and these different versions of reconnection may form similar magnetic perturbations by very different ways. A clear example is the bipolar signatures in $B_{z}$ associated with BBFs which is discussed in Sects. 2.1, $2.2,2.4$ and 2.5. A consequence is that such variations, which have appeared under different names, may even be the manifestation of the same process. Hence, although it might be tempting, searching for a single consistent picture may be futile. It is also not the purpose of this paper to promote a particular interpretation but to recognize that several different manifestations of the processes lead to a multitude of possible signatures. A caution would be that one should not rely exclusively on the magnetic field perturbation analysis (as often is the case due to practical reasons) to identify the generation mechanism.

Another obvious reason for the complexity is that the separation of spatial and temporal components of the variations is still more of an art than a routine procedure. A clear example is the observation of the PSBL, whose position is influenced by the variations of the reconnection rate, by other types of the boundary motions (e.g., kink-like flapping oscillation etc.) and by field-aligned dynamics of particle beam and electromagnetic waves, and where reliable separation of temporal and spatial origins is not yet achieved. For example, accelerated ion beams at the plasma sheet-lobe interface are always presented in terms of cross cuts of their spatialtemporal pattern along the spacecraft trajectory and no standard way exists to separate between TDIS, VDIS (Sect. 2.9) or place of birth effects from their mixture. Cluster observations open new possibilities for such studies, but one should recognize that much more work needs to be done to fully exploit the possibilities of multi-spacecraft analysis.

The extensive Cluster data need to be analyzed in a more quantitative way. Simple cartoons and simple static paradigms have very limited significance in the application to transient localized mesoscale structures. For example, even the simplest version of transient reconnection model predicts not only perturbation timing and amplitude variations in space, but also the characteristic waveform (e.g., bipolar or unipolar $B_{z}$ variation, or one or two pulse appearance in $B_{x}$ and $B_{z}$ waveforms in Fig. 31) depends on distances from the source (which are always not known). Correspondingly, our conclusions about the source of perturbations should be based on the spatial gradient information and quantitative relationships among different physical parameters. Several new advanced quantitative tools are being developed based on reconstruction analysis technique to obtain reconnection rate (Sect. 3.1) and topology/scale size of the plasma structures (Sect. 3.4), and these can predict the characteristics of the source in a larger context. Using these new tools and using the known separations of the spacecraft, more quantitative multi-point event analysis can characterize plasma boundaries and their temporal evolution. This is essential for further understanding of the transients in the magnetotail and to reveal the relationships between the different physical mechanisms and their signatures. Thus, the future studies require (1) the use (and extension) of quantitative methods utilizing multipoint observations, and (2) stronger interaction between data analysis and simulations of meso-scale structures in order to test available methods and to enrich the physical models.

Transient processes produced by reconnection and other instabilities have been considered also to play an important role in the dayside magnetopause and flanks. Recent advances in these areas based on Cluster observations are reviewed by Paschmann et al. (2005). It should be noted that although these dayside/flank processes are not directly coupled to the nightside transients themselves, they can provide essential background conditions for the transient processes discussed in this paper. Examples are the Kelvin-Helmholtz waves and reconnection along the tail flanks, providing additional entry mechanism of the solar wind plasma affecting the preconditioning of the plasma sheet. Such preconditioning of the plasma sheet is set also by the energy/charge dependent motion of the particles, causing a natural dawn-dusk asymmetry in the plasma sheet dynamics. Thus, to understand the nightside transient processes in the context of larger scale magnetospheric dynamics, further multi-point missions are needed.

\section{Summary}

The mesoscale phenomena reviewed in this paper provide the main links between the microscale processes responsible for the initiation of magnetospheric phenomena and their large scale manifestations. The connection between the micro- and macro-scales take place through many paths and magnetic reconnection is one of the common threads between bursty bulk flows, traveling compression regions, flux ropes, night side flux transfer events and transient field-aligned currents. These phenomena provide important means for studying reconnection in collisionless plasmas, including the reconnection rate and associated current system. The magnetotail current sheet exhibits dynamical features, such as flapping and oscillations, and its evolution or disruption are crucial to the understanding of magnetospheric phenomena. The acceleration and energization of particles accompanying the sudden 
release of magnetic energy take place during magnetic reconnection and in thin current sheets. The energetic particles observed in the near-Earth tail region can however originate from the non-adiabatic acceleration in current sheets beyond the reconnection region and then propagate along the field lines as highly structured beamlets.

The new advances, mainly enabled by the Cluster observations, underlines the important role of multi-point measurements. Furthermore, the Magnetospheric Multiscale (MMS) mission will explore the whole range of microscale processes associated with magnetic reconnection on scales down to the electron gyroradius. On the broader scale, the THEMIS (Time History of Events and Macroscale Interactions during Substorms) will investigate the large-scale magnetotail and substorm dynamics and solar wind - magnetosphere coupling, thus specifying the wider context of the magnetospheric processes.

Acknowledgements. The authors thank the International Space Science Institute (ISSI, Bern, Switzerland) for making this review possible by funding two meetings of an international team of scientists (ISSI Team 91). We are grateful to the ISSI staff for their excellent support during the team meetings. The research at the participating institutions are supported by many grants: NASA Cluster Theory Guest Investigator grant NNG-05-GG26G to Dartmouth College; NASA Geospace Science grants (NNG-04-GE37G and NNX-07AF42G) and NSF grant DMS 0417800 to University of Maryland, College Park; NASA contract NAS5-02099 to UCLA; RFBR grants 06-05-90631, 07-02-91703, 07-02-00319 and 06-02-72561 to Institute for Space Research, Moscow; 07-05-00776, 07-05-91703, and 2190 (Intergeophysics) to St. Petersburg University, St. Petersburg. The team at CESR/CNRS, Toulouse thanks CNRS, CNES and Paul Sabatier University for support.

Topical Editor I. A. Daglis thanks two anonymous referees for their help in evaluating this paper.

\section{References}

Alexeev, I. V., Owen, C. J., Fazakerley, A. N., Runov, A., Dewhurst, J. P., Balogh, A., Rème, H., Klecker, B., and Kistler, L.: Cluster observations of currents in the plasma sheet during reconnection, Geophys. Res. Lett., 32, L03101, doi:10.1029/2004GL021420, 2005.

Alexeev, I. V., Sergeev, V., Owen, C. J., Fazakerley, A., Lucek, E., and Rème, H.: Remote sensing of a magnetotail reconnection Xline using polar rain electrons, Geophys. Res. Lett., 33, L19105, doi:10.1029/2006GL027243, 2006.

Amm, O., Nakamura, R., Frey, H. U., Ogawa, Y., Kubyshkina, M., Balogh, A., and Rème, H.: Substorm topology in the ionosphere and magnetosphere during a flux rope event in the magnetotail, Ann. Geophys., 24, 735-750, 2006, http://www.ann-geophys.net/24/735/2006/.

Angelopoulos, V., Baumjohann, W., Kennel, C. F., Coroniti, F. V., Kivelson, M. G., Pellat, R., Walker, R. J., Lühr, H., and Paschmann, G.: Bursty bulk flows in the inner central plasma sheet, J. Geophys. Res., 97, 4027-4039, 1992.

Angelopoulos, V., Kennel, C. F., Coroniti, F. V., Pellat, R., Kivelson, M. G., Walker, R. J., Russell, C. T., Baumjohann, W., Feld- man, W. C., and Gosling, J. T.: Statistical characteristics of bursty bulk flow events, J. Geophys. Res., 99, 21 257-21 280, 1994.

Angelopoulos, V., Phan, T. D., Larson, D. E., et al.: Magnetotail flow bursts: association to global magnetospheric circulation, relationship to ionospheric activity and direct evidence for localization, Geophys. Res. Lett., 24, 2271-2274, 1997.

Ashour-Abdalla, M., Berchem, J. P., Buchner, J., and Zelenyi, L. M.: Shaping of the magnetotail from the mantle: Global and local structuring, J. Geophys. Res., 98, 5651-5676, 1993.

Baker, D. N. and Stone, E. C.: Energetic electron anisotropies in the magnetotail - Identification of open and closed field lines, Geophys. Res. Lett., 3, 557-560, 1976.

Baker, D. N. and Stone, E. C.: Observations of energetic electrons E no less than about $200 \mathrm{keV}$ in the Earth's magnetotail: Plasma sheet and fireball observations, J. Geophys. Res., 82, 1532-1546, 1977.

Baker, D. N., Pulkkinen, T. I., Angelopoulos, V., Baumjohann, W., and McPherron, R. L.: Neutral line model of substorms: Past results and present view, J. Geophys. Res., 101, 12 975-13010, 1996.

Bauer, T. M., Baumjohann, W., Treumann, R. A., Sckopke, N., and Lühr, H.: Low-frequency waves in the near-Earth plasma sheet, J. Geophys. Res., 100, 9605-9618, 1995.

Baumjohann, W.: The near-Earth plasma sheet: An AMPTEE/IRM perspective, Space Sci. Rev., 64, 141-163, 1993.

Baumjohann, W., Paschmann, G., and Lühr, H.: Characteristics of high-speed flows in the plasma sheet, J. Geophys. Res., 95, 3801-3809, 1990.

Belehaki, A. and Tsagouri, I.: Magnetosphere energetics during substorm events: IMP 8 and GEOTAIL observations, J. Atmos. Terr. Phys., 63, 657-670, 2001.

Biernat, H. K., Heyn, M. F., and Semenov, V. S.: Unsteady Petschek reconnection, J. Geophys. Res., 92, 3392-3396, 1987.

Birn, J.: Simulations of reconnection in the magnetotail, in: Reconnection of Magnetic fields, edited by: Birn, J. and Priest, E., pp. 201-208, Cambridge Univ. Press, Cambridge, 2007.

Birn, J. and Hesse, M.: Details of current disruption and diversion in simulations of magnetotail dynamics, J. Geophys. Res., 101, 15345-15358, 1996.

Birn, J., Hesse, M., and Schindler, K.: Filamentary structure of a three-dimensional plasmoid, J. Geophys. Res., 94, 241-251, 1989.

Birn, J., Drake, J., Shay, M. A., Rogers, B. N., Denton, R. E., Hesse, M., Kuznetsova, M., Ma, Z. W., Bhattacharee, A., Otto, A., Prichett, P. L.: Geospace Environment Modeling (GEM) Magnetic reconnection challenge, J. Geophys. Res., 106, 3715-3719, 2001.

Birn, J., Raeder, J., Wang, Y. L., Wolf, R. A., and Hesse, M.: On the propagation of bubbles in the geomagnetic tail, Ann. Geophys., 22, 1773-1786, 2004, http://www.ann-geophys.net/22/1773/2004/.

Birn, J., Hesse, M., and Schindler, K.: On the role of entropy conservation and entropy loss governing substorm phases, in: Proceedings of the Eght International Conference on Substorms, edited by: Syrjasuo, M. and Donovan, E., pp. 19-24, University of Calgary, Calgary, Canada, 2006.

Borälv, E., Opgenoorth, H. J., Kauristie, K., Lester, M., Bosqued, J.-M., Dewhurst, J. P., Owen, C. J., Dunlop, M., Slavin, J. A., 
Fazakerley, A., and Perry, C.: Correlation between ground-based observations of substorm signatures and magnetotail dynamics, Ann. Geophys., 23, 997-1011, 2005, http://www.ann-geophys.net/23/997/2005/.

Borovsky, J. E. and Funsten, H. O.: MHD turbulence in the Earth's plasma sheet: Dynamics, dissipation, and driving, J. Geophys. Res., 108, 1284, doi:10.1029/2002JA009625, 2003.

Borovsky, J. E., Elphic, R. C., Funsten, H. O., and Thomsen, M. F.: The Earth's plasma sheet as a lobaratory for flow turbulence in high- $\beta$ MHD, J. Plasma Phys., 57, 1-34, 1997.

Büchner, J. and Zelenyi, L. M.: Regular and chaotic charged particle motion in magnetotaillike field reversals, 1, Basic theory of trapped motion, J. Geophys. Res., 94, 11 821-11 842, 1989.

Burkhart, G. R. and Chen, J.: Differential memory in the Earth's magnetotail, J. Geophys. Res., 96, 14,033, 1991.

Burlaga, L. F.: Magnetic Clouds and Force free fields with constant alpha, J. Geophys. Res., 93, 7217-7224, 1988.

Cao, J. B., Ma, Y. D., Parks, G., Rème, H., Dandouras, I., Nakamura, R., Zhang, T. L., Zong, Q., Lucek, E., Carr, C. M., Liu, Z. X., and Zhou, G. C.: Joint observations by Cluster satellites of bursty bulk flows in the magnetotail, J. Geophys. Res., 111, A04206, doi:10.1029/2005JA011322, 2006.

Cattell, C., Mozer, F., Tsuruda, K., Hayakawa, H., Nakamura, M., Okada, T., Kokubun, S., and Yamamoto, T.: Geotail observations of spiky electric fields and low-frequency waves in the plasma sheet and plasma sheet boundary, Geophys. Res. Lett., 21, 29872990, 1994.

Cattell, C., Dombeck, J., Wygant, J., Drake, J. F., Swisdak, M., Goldstein, M. L., Keith, W., Fazakerley, A., André, M., Lucek, E., and Balogh, A.: Cluster observations of electron holes in association with magnetotail reconnection and comparison to simulations, J. Geophys. Res., 110, A01211, doi: 10.1029/2004JA010519, 2005.

Chandresakhar, S. and Woltjer, L.: On Force-Free Magnetic Fields, Proc. Natl. Acad. Sci. USA, 44(4), 285-289, 1958.

Chanteur, G. and Harvey, C. C.: Spatial interpolation for four spacecraft: Application to magnetic gradients, in: Analysis Methods for Multi-Spacecraft Data, edited by: Paschmann, G. and Daly, P., pp. 349-369, ESA, Noordwijk, 1998.

Chen, C. X. and Wolf, R. A.: Theory of thin filament motion in Earth's magnetotail and its application to bursty bulk flows, J. Geophys. Res., 104, 14 613-14 626, 1999.

Consolini, G., Kerzschmar, M., Lui, A. T. Y., Zimbardo, G., and Macek, W. M.: On the magnetic field fluctuations during magnetospheric tail current disruption: a statistical approach, J. Geophys. Res., 110, A07202, doi:10.1029/2004JA010947, 2005.

Coroniti, F. V.: Laminar wave-train structure of collisionless magnetic slow shocks, Nucl. Fusion, 11, 261-283, 1971.

Coroniti, F. V.: Explosive tail reconnection - The growth and expansion phases of magnetospheric substorms, J. Geophys. Res., 90, 7427-7447, 1985.

Cowley, S. W. H.: Asymmetry effects associated with the $\mathrm{x}-$ component of the IMF in a magnetically open magnetosphere, Planet. Space Sci., 29, 809-818, 1981.

Dahlburg, R. B., Antiochos, S. K., and Zang, T. A.: Secondary instability in three-dimensional magnetic reconnection, Phys. Fluids B, 4, 3902-3914, 1992.

Daughton, W.: Nonlinear dynamics of thin current sheets, Phys. Plasmas, 9, 3668-3678, 2002.
DeCoster, R. J. and Frank, L. A.: Observations pertaining to the dynamics of the plasma sheet, J. Geophys. Res., 84, 5099-5121, 1979.

Delcourt, D. C., Sauvaud, J.-A., Vaisberg, O. L., Avanov, L. A., Burch, J. L., and White, J. H.: Signatures of impulsive convection in the magnetospheric lobes, Geophys. Res. Lett., 23, 129132, 1996

Drake, J. F., Swisdak, M., Cattell, C., Shay, M. A., Rogers, B. N., and Zeiler, A.: Formation of Electron Holes and Particle Energization During Magnetic Reconnection, Science, 299, 873-877, 2003.

Drake, J. F., Swisdak, M., Schoeffler, K. M., Rogers, B. N., and Kobayashi, S.: Formation of secondary islands during magnetic reconnection, Geophys. Res. Lett., 33, L13105, doi:10.1029/ 2006GL025957, 2006.

Dunlop, M. W. and Woodward, T. I.: Cluster Magnetic Field Analysis Techniques, in: Cluster-II Workshop: Multiscale/Multipoint Plasma Measurements, Proceedings of the Workshop held at Imperial College, London, UK, 22-24 September 1999. Paris: European Space Agency (ESA), 2000, ESA-SP, vol. 449, ISBN: 9290927968, p.351, edited by: Harris, R. A., p. 351, 2000.

Eastman, T. E., Frank, L. A., Peterson, W. K., and Lennartsson, W.: The plasma sheet boundary layer, J. Geophys. Res., 89, 15531564, 1984.

Eastwood, J. P., Sibeck, D. G., Slavin, J. A., Goldstein, M. L., Lavraud, B., Sitnov, M., Imber, S., Balogh, A., Lucek, E. A., and Dandouras, I.: Observations of multiple X-line structure in the Earth's magnetotail current sheet: A Cluster case study, Geophys. Res. Lett., 32, L11105, doi:10.1029/2005GL022509, 2005.

Elphic, R. C., Mutch, P. A., and Russell, C. T.: Observations of field-aligned currents at the plasma sheet boundary: An ISEE-1 and 2 survey, Geophys. Res. Lett., 12, 631-634, 1985.

Elphic, R. C., Russell, C. T., Cattell, C. A., Takahasi, K., and Bame, S. J.: ISEE-1 and 2 observations of magnetic flux ropes in the magnetotail - FTE's in the plasma sheet?, Geophys. Res. Lett., 13, 648-651, 1986.

Elphic, R. C., Onsager, T. G., Thomsen, M. F., and Gosling, J. T.: Nature and location of the source of plasma sheet boundary layer ion beams, J. Geophys. Res., 100, 1857-1869, 1995.

Erickson, G. M. and Wolf, R. A.: Is steady convection possible in the Earth's magnetotail, Geophys. Res. Lett., 7, 897-900, 1980.

Eriksson, S., Öieroset, M., Baker, D. N., Mouikis, C., Vaivads, A., Dunlop, M. W., Rème, H., Ergun, R. E., and Balogh, A.: Walén and slow mode shock analyses in the near-Earth magnetotail in connection with a substorm onset on 27 August 2001, J. Geophys. Res., 109, A05212, doi:10.1029/2004JA010534, 2004.

Escoubet, C. P., Schmidt, R., and Goldstein, M. L.: Cluster - Science and Mission Overview, Space Sci. Rev., 79, 11-32, 1997.

Escoubet, C. P., Fehringer, M., and Goldstein, M. L.: The Cluster mission, Ann. Geophys., 19, 1197-1200, 2001, http://www.ann-geophys.net/19/1197/2001/.

Feldman, W. C., Bame, S. J., Baker, D. N., Birn, J., Gosling, J. T., Hones, Jr., E. W., McComas, D. J., Zwickl, R. D., Slavin, J. A., and Smith, E. J.: Evidence for slow-mode shocks in the deep geomagnetic tail, Geophys. Res. Lett., 11, 599-602, 1984.

Feldman, W. C., Tokar, R. L., Birn, J., Hones Jr., E. W., Bame, S. J., and Russell, C. T.: Structure of a slow mode shock observed in the plasma sheet boundary layer, J. Geophys. Res., 92, 83-94, 1987. 
Frank, L. A., Paterson, W. R., Ackerson, K. L., Kokubun, S., Yamamoto, T., Fairfield, D. H., and Lepping, R. P.: Observations of plasmas associated with the magnetic signature of a plasmoid in the distant magnetotail, Geophys. Res. Lett., 21, 2967-2970, 1994.

Frank, L. A., Paterson, W. R., Kokubun, S., Yamamoto, T., and Lepping, R. P.: Direct detection of the current in a magnetotail flux rope, Geophys. Res. Lett., 22, 2697-2700, 1995.

Freeman, M. P., Watkins, N. W., and Riley, D. J.: Evidence for a solar wind origin of the power law burst lifetime distribution of the AE indices, Geophys. Res. Lett., 27, 1087-1090, 2000.

Fruit, G., Louarn, P., Tur, A., and Quéau, D. L.: On the propagation of magnetohydrodynamic perturbations in a Harris-type current sheet 1. Propagation on discrete modes and signal reconstruction, J. Geophys. Res., 107, 1411, doi:10.1029/2001JA009212, 2002a.

Fruit, G., Louarn, P., Tur, A., and Quéau, D. L.: On the propagation of magnetohydrodynamic perturbations in a Harris-type current sheet: 2. Propagation on continuous modes and resonant absorption, J. Geophys. Res., 107, 1412, doi:10.1029/2001JA009215, $2002 b$.

Fruit, G., Louarn, P., Budnik, E., Sauvaud, J., Jacquey, C., Le Queau, D., Reme, H., Lucek, E., Balogh, A., and CornilleauWehrlin, N.: On the propagation of low frequency fluctuations in the plasma sheet: 2. Characterization of the MHD eigenmodes and physical implications, J. Geophys. Res., 109, A03217, doi: 10.1029/2003JA10229, 2004.

Fujimoto, M., Nagai, T., Yokokawa, N., Yamade, Y., Mukai, T., Saito, Y., and Kokubun, S.: Tailward electrons at the lobe-plasma sheet interface detected upon dipolarizations, J. Geophys. Res., 106, 21 255-21 262, 2001.

Garner, T. W., Wolf, R. A., Spiro, R. W., Thomsen, M. F., and Korth, H.: Pressure balance inconsistency exhibited in a statistical model of magnetospheric plasma, J. Geophys. Res., 108, 1331, doi:10.1029/2003JA009877, 2003.

Golovchanskaya, I. V. and Maltsev, Y. P.: Interchange instability in the presence of the field-aligned currents: Application to the auroral arc formation, J. Geophys. Res., 108, 1106, doi:10.1029/ 2002JA009505, 2003.

Golovchanskaya, I. V. and Maltsev, Y. P.: On the identification of plasma sheet flapping waves observed by Cluster, Geophys. Res. Lett, 32, L02102, doi:10.1029/2004GL021552, 2004.

Gombosi, T., De Zeeuw, D. L., Powell, K. G., Ridley, A. J., Sokolov, I. V., Stout, Q. F., Toth, G.: Adaptivr Mesh Refinement MHD for Global Space Weather Simulations, in: Space Plasma Simulations, edited by: Buechner, J., Dum, C., and Scholer, M., pp. 251-279, Springer, Berlin, 2003.

Grocott, A., Yeoman, T. K., Nakamura, R., Cowley, S. W. H., Frey, H. U., Rème, H., and Klecker, B.: Multi-instrument observations of the ionospheric counterpart of a bursty bulk flow in the nearEarth plasma sheet, Ann. Geophys., 22, 1061-1075, 2004, http://www.ann-geophys.net/22/1061/2004/.

Gurnett, D. A., Frank, L. A., and Lepping, R. P.: Plasma waves in the distant magnetotail, J. Geophys. Res., 81, 6059-6071, 1976.

Harvey, C. C.: Spatial gradients and volumetric tensor, in: Analysis Methods for Multi-Spacecraft Data, edited by: Paschmann, G. and Daly, P., pp. 307-322, ESA, Noordwijk, 1998.

Hasegawa, H., Sonnerup, B. U. Ö., Klecker, B., Paschmann, G., Dunlop, M. W., and Rème, H.: Optimal reconstruction of magnetopause structures from Cluster data, Ann. Geophys., 23, 973-
982, 2005,

http://www.ann-geophys.net/23/973/2005/.

Hau, L.-N. and Sonnerup, B. U. O.: The structure of resistivedispersive intermediate shocks, J. Geophys. Res., 95, 1879118 808, 1990.

Hau, L.-N. and Sonnerup, B. U. O.: The thickness of resistivedispersive shocks, J. Geophys.Res., 97, 8269-8275, 1992.

Hau, L.-N. and Sonnerup, B. U. Ö.: Two-dimensional coherent structures in the magnetopause: Recovery of static equilibria from single-spacecraft data, J. Geophys. Res., 104, 6899-6917, 1999.

Henderson, P. D., Owen, C. J., Alexeev, I. V., Slavin, J., Fazakerley, A. N., Lucek, E., and Rème, H.: Cluster observations of flux rope structures in the near-tail, Ann. Geophys., 24, 651-666, 2006a, http://www.ann-geophys.net/24/651/2006/.

Henderson, P. D., Owen, C. J., Lahiff, A. D., Alexeev, I. V., Fazakerley, A. N., Lucek, E., and Rème, H.: Cluster PEACE observations of electron pressure tensor divergence in the magnetotail, Geophys. Res. Lett., 33, L22106, doi:10.1029/2006GL027868, 2006b.

Hesse, M. and Kivelson, M. G.: The Formation and Structure of Flux Ropes in the Magnetotail, in: New Perspectives on the Earth's Magnetotail, edited by: Nishida, A., Baker, D. N., and Cowley, S. W. H., p. 139, 1998.

Hesse, M. and Winske, D.: Hybrid simulations of collison-less reconnection in current sheets, J. Geophys. Res., 99, $11177-$ $11192,1994$.

Hesse, M., Birn, J., Baker, D. N., and Slavin, J. A.: MHD simulations of the transition of magnetic reconnection from closed to open field lines, J. Geophys. Res., 101, 10 805-10 816, 1996.

Heyn, M. F. and Semenov, V. S.: Rapid reconnection in compressible plasma, Phys. Plasmas, 3, 2725-2741, 1996.

Heyn, M. F., Biernat, H. K., Rijnbeek, R. P., and Semenov, V. S.: The structure of reconnection layers, J. Plasma Physics, 40, 235252, 1988.

Hirahara, M., Mukai, T., Nagai, T., Kaya, N., Hayakawa, H., and Fukunishi, H.: Two types of ion energy dispersions observed in the nightside auroral regions during geomagnetically disturbed periods, J. Geophys. Res, 101, 7749-7768, 1996.

Hnat, B., Chapman, S. C., Rowlands, G., Watkins, N. W., and Freeman, M. P.: Scaling in long term data sets of geomagnetic indices and solar wind $\epsilon$ as seen by WIND spacecraft, Geophys. Res. Lett., 30, 2174, doi:10.1029/2003GL018209, 2003.

Hones, E. W.: Substorm processes in the magnetotail: Comments on "On hot tenuous plasmas, fireballs, and boundary layers in the Earth's magnetotail” by Frank et al., J. Geophys. Res., 82, 5633-5643, 1977.

Hones, E. W., Higbie, P. R., and Palmer, I. D.: Energetic protons of magnetospheric origin in the plasma sheet associated with substorms, J. Geophys. Res., 81, 3866-3874, 1976.

Hones, E. W., Baker, D. N., Bame, S. J., Feldman, W. C., Gosling, J. T., McComas, D. J., Zwickl, R. D., Slavin, J. A., Smith, E. J., and Tsurutani, B. T.: Structure of the magnetotail at 220 Earth radii and its response to geomagnetic activity, Geophys. Res. Lett., 11, 5-7, 1984.

Hoshino, M.: Electron surfing acceleration in magnetic reconnection, J. Geophys. Res., 110, A10215, doi:10.1029/ 2005JA011229, 2005.

Hoshino, M., Mukai, T., Nishida, A., Kokubun, S., and Yamamoto, 
T.: Non-Gyrotropic Ions as Evidence for An X-Type Neutral Region, Adv. Space Res., 26, 425-430, 2000.

Hoshino, M., Mukai, T., Terasawa, T., and Shinohara, I.: Superthermal electron acceleration in magnetic reconnection, J. Geophys. Res., 106, 25 972-25 997, 2001.

Huang, C.-S., Foster, J. C., Reeves, G. D., Le, G., Frey, H. U., Pollock, C. J., and Jahn, J.-M.: Periodic magnetospheric substorms: Multiple space-based and ground-based instrumental observations, J. Geophys. Res., 108, 1411, doi:10.1029/2003JA009992, 2003.

Huba, J. D., Drake, J. F., and Gladd, N. T.: Lower-hibrid-drift instability in field reversed plasmas, Phys. Fluids, 23, 552-561, 1980.

Hughes, W. J. and Sibeck, D. G.: On the 3-dimensional structure of plasmoids, Geophys. Res. Lett., 14, 636-639, 1987.

Hultqvist, B., Oieroset, M., Paschmann, G., and Treumann, R.: Magnetospheric Plasma Sources and Losses, pp. 1-483, Kluwer Academic Publisher, the Netherland, 1999.

Ieda, A., Machida, S., Mukai, T., Saito, Y., Yamamoto, T., Nishida, A., Terasawa, T., and Kokubun, S.: Statistical analysis of the plasmoid evolution with Geotail observations, J. Geophys. Res., 103, 4453-4466, 1998.

Ieda, A., Fairfield, D. H., Mukai, T., Saito, Y., Kokubun, S., Liou, K., Meng, C.-I., Parks, G. K., and Brittnacher, M. J.: Plasmoid ejection and auroral brightenings, J. Geophys. Res., 106, 38453857, 2001.

Iijima, T. and Potemra, T. A.: Large-scale characteristics of fieldaligned currents associated with substorms, J. Geophys. Res., 83, 599-615, 1978.

Imada, S., Hoshino, M., and Mukai, T.: Average profiles of energetic and thermal electrons in the magnetotail reconnection regions, Geophys. Res. Lett., 32, L09101, doi:10.1029/ 2005GL022594, 2005.

Imada, S., Nakamura, R., Daly, P. W., Hoshino, M., Baumjohann, W., Mühlbachler, S., Balogh, A., and Rème, H.: Energetic electron acceleration in the downstream reconnection outflow region, J. Geophys. Res., 112, A03202, doi:10.1029/2006JA011847, 2007.

Jacquey, C., Sauvaud, J.-A., and Dandouras, J.: Location and propagation of the magnetotail current disruption during substorm expansion: analysis and simulation of ISEE multi-onset event, Geophys. Res. Lett., 18, 389-392, 1991.

Jacquey, C., Sauvaud, J.-A., Dandouras, J., and Korth, A.: Tailward propagating cross-tail current disruption and dynamics of nearEarth tail: Multi-point measurement analysis, Geophys. Res. Lett., 20, 983-986, 1993.

Jacquey, C., Sauvaud, J. A., Popescu, D., Reme, H., Sibeck, D. G., Klimov, S. I., Romanov, S. A., Lepping, R. P., and Reeves, G. D.: Large scale response of the magnetotail to a substorm expansion: Interball and IMP-8 observations on November 24 1996, in: Substorms-4, edited by: Kokubun, S. and Kamide, Y., pp. 155-158, Kluwer Academic Publishers, 1998.

Karimabadi, H., Pritchett, P. L., Daughton, W., and KraussVarban, D.: Ion-ion kink instability in the magnetotail: 2 Threedimensional full particle and hybrid simulations and comparison with observations, J. Geophys. Res., 108, 1401, doi:10.1029/ 2003JA010109, 2003.

Katsouleas, T. and Dawson, J. M.: Unlimited Electron Acceleration in Laser-Driven Plasma Waves, Phys. Rev. Lett., 51, 846, 1983.

Kauristie, K., A.Sergeev, V., Kubyshkina, M., Pulkkinen, T. I., An- gelopoulos, V., Phan, T., Lin, R. P., and Slavin, J. A.: Ionospheric current signatures of transient plasma sheet flows, J. Geophys. Res., 105, 10 677-25 904, 2000.

Kauristie, K., Sergeev, V. A., Amm, O., Kubyshkina, M. V., Jussila, J., Donovan, E., and Liou, K.: Bursty bulk flow intrusion to the inner plasma sheet as inferred from auroral observations, J. Geophys. Res., 108, 1040, doi:10.1029/2002JA009371, 2003.

Kawano, H., Yamamoto, T., Kokubun, S., Tsuruda, K., Lui, A. T. Y., Williams, D. J., Yumoto, K., Hayakawa, H., Nakamura, M., and Okada, T.: A flux rope followed by recurring encounters with traveling compression regions: GEOTAIL observations, Geophys. Res. Lett., 21, 2891-2894, 1994.

Kazama, Y. and Mukai, T.: Simultaneous appearance of energydispersed ion signatures of ionospheric and magnetotail origins in the near-Earth plasma sheet, J. Geophys. Res., 110, A07213, doi:10.1029/2004JA010820, 2005.

Keiling, A., Rème, H., Dandouras, I., Bosqued, J. M., Parks, G. K., McCarthy, M., Kistler, L., Amata, E., Klecker, B., Korth, A. and Lundin, R.: Transient ion beamlet injections into spatially separated PSBL flux tubes observed by Cluster-CIS, Geophys. Res. Lett., 31, L12804, doi:10.1029/2004GL020192, 2004.

Keiling, A., Parks, G. K., Rème, H., Dandouras, I., Wilber, M., Kistler, L., Owen, C., Fazakerley, A. N., Maksimovic, E. L. M., and Cornilleau-Wehrlin, N.: Energy-dispersed ions in the plasma sheet boundary layer and associated phenomena: Ion heating, electron acceleration, Alfvén waves, broadband waves, perpendicular electric field spikes, and auroral emissions, Ann Geophys., 24, 2685-2707, 2006.

Kennel, C. F.: Convection and Substorms, Oxford University Press, 1995.

Kepko, L. and Kivelson, M.: Generation of Pi2 pulsations by bursty bulk flows, J. Geophys. Res., 104, 25 021-25 034, 1999.

Khurana, K. K., Kivelson, M. G., Frank, L. A., and Paterson, W. R.: Observations of magnetic flux ropes and associated currents in Earth's magnetotail with the Galileo spacecraft, Geophys. Res. Lett., 22, 2087-2090, 1995.

Kivelson, M. G., Kennel, C. F., McPherron, R. L., Russell, C. T., Southwood, D. J., Walker, R. J., Khurana, K. K., Coleman, P. J., Hammond, C. M., and Angelopoulos, V.: The Galileo Earth encounter - Magnetometer and allied measurements, J. Geophys. Res., 98, 11 299-11318, 1993.

Kojima, H., Matsumoto, H., Miyatake, T., Nagano, I., Fujita, A., Frank, L. A., Mukai, T., Paterson, W. R., Saito, Y., and Machida, S.: Relation between electrostatic solitary waves and hot plasma flow in the plasma sheet boundary layer: GEOTAIL observations, Geophys. Res. Lett., 21, 2919-2922, 1994.

Lapenta, G. and Brackbill, J. U.: A kinetic theory for the drift-kink instability, J. Geophys. Res., 102, 27 099-27 108, 1997.

Lee, L. C.: A Review of Magnetic Reconnection: MHD Models, p. 139-153, Phys. Magnetopause, 1995.

Lee, L. C. and Fu, Z. F.: A theory of magnetic flux transfer at the Earth's magnetopause, Geophys. Res. Lett., 12, 105-108, 1985.

Lee, L. C., Wang, S., Wei, C. Q., and Tsurutani, B. T.: Streaming sausage, kink and tearing instabilities in a current sheet with application to the Earth's magnetotail, J. Geophys. Res., 93, 73547365, 1988.

Lepping, R. P., Burlaga, L. F., and Jones, J. A.: Magnetic field structure of interplanetary magnetic clouds at 1 AU, J. Geophys. Res., 95, 11 957-11965, 1990. 
Lepping, R. P., Fairfield, D. H., Jones, J., Frank, L. A., Paterson, W. R., Kokubun, S., and Yamamoto, T.: Cross-tail magnetic flux ropes as observed by the GEOTAIL spacecraft, Geophys. Res. Lett., 22, 1193-1196, 1995.

Lopez, R. E. and Lui, A. T. Y.: A multisatellite case study of the expansion of a substorm current wedge in the near-Earth magnetotail, J. Geophys. Res., 95, 8009-8017, 1990.

Louarn, P., Fruit, G., Budnik, E., Sauvaud, J.-A., Jacquey, C., Quéau, D. L., Rème, H., Lucek, E., Balogh, A., et al.: On the propagation of low frequency fluctuations in the plasmasheet: 1 . CLUSTER observations and magnetohydrodynamic analysis, J. Geophys. Res., 109, A03216, doi:10.1029/2003JA010228, 2004.

Lui, A. T. Y.: Characteristics of the cross-tail current in the Earth's magnetotail, in: Magnetospheric Currents, AGU Geophysical Monograph 28, edited by: Potemra, T., pp. 158-170, AGU, Washington, D.C., 1984.

Lui, A. T. Y.: Multiscale phenomena in the near-Earth magnetosphere, J. Atmos. Sol. Terr. Phys., 64, 125-143, 2002.

Lui, A. T. Y.: Current disruption in the Earth's magnetosphere: Observations and models, J. Geophys. Res., 101, 13067-13088, 1996.

Lui, A. T. Y., Hones, Jr, E. W., Yasuhara, F., Akasofu, S.-I., and Bame, S. J.: Magnetotail plasma flow during plasma sheet expansions: VELA 5 and 6 and IMP 6 observations, J. Geophys. Res., 82, 1235-1247, 1977.

Lui, A. T. Y., Meng, C.-I., and Akasofu, S.-I.: Wavy nature of the magnetotail neutral sheet, Geophys. Res. Lett., 5, 279-282, 1978.

Lui, A. T. Y., Brittnacher, M. J., Christon, S. P., Eastman, T. E., Kokubun, S., Liou, K., McEntire, R. W., Meng, C.-I., Newell, P. T., Parks, G. K., Yamamoto, T., and Williams, D. J.: Ionospheric current signatures of magnetic flux rope in the magnetotail, Geophys. Res. Lett., 19, 3733-3736, 1998.

Lyon, J.G.: The Solar Wind-Magnetosphere-Ionosphere System, Science, 288, 1987-1991, 2000.

Lyons, L. R. and Speiser, T. W.: Evidence for current sheet acceleration in the geomagnetic tail, J. Geophys. Res., 87, 2276-2286, 1982.

Maezawa, K.: Magnetotail boundary motion associated with geomagnetic substorms, J. Geophys. Res., 80, 3543-3548, 1975.

Malova H. V., Zelenyi, L. M., Popov, V. Y., Delcourt, D. C., Petrukovich, A. A., and Runov, A.: Asymmetric thin current sheets in the Earth's magnetotail, Geophys. Res. Lett., 34, L16108, doi:10.1029/2007GL030011, 2007.

Möbius, E., Scholer, M., Hovestadt, D., Paschmann, G., and Glöckler, G.: Energetic particles in the vicinity of a possible neutral line in the plasma sheet, J. Geophys. Res., 88, 7742-7752, 1983.

Moldwin, M. B. and Hughes, W. J.: Plasmoids as magnetic flux ropes, J. Geophys. Res., 96, 14 051-14 064, 1991.

Moldwin, M. B. and Hughes, W. J.: Multi-satellite observations of plasmoids - IMP 8 and ISEE 3, Geophys. Res. Lett., 19, 10811084, 1992.

Moldwin, M. B. and Hughes, W. J.: Observations of Earthward and tailward propagating flux rope plasmoids: Expanding the plasmoid model of geomagnetic substorms, J. Geophys. Res., 99, 183-198, 1994.

Mukai, T., Fujimoto, M., Kokubun, S., Machida, S., Maezawa, K., Nishida, A., Saito, Y., Terasawa, T., and Yamamoto, T.: Structure and kinetic properties of plasmoids and their boundary regions, J. Geomag. Geoelectr., 48, 541-560, 1996.

Murphy, N., Slavin, J. A., Baker, D. N., and Hughes, W. J.: Enhancements of energetic ions associated with travelling compression regions in the deep geomagnetic tail, J. Geophys. Res., 92, 64-70, 1987.

Nagai, T., Takahashi, K., Kawano, H., Yamamoto, T., Kokubun, S., and Nishida, A.: Initial GEOTAIL survey of magnetic substorm signatures in the magnetotail, Geophys. Res. Lett., 21, 29912994, 1994.

Nagai, T., Fujimoto, M., Saito, Y., Machida, S., Terasawa, T., Nakamura, R., Yamamoto, T., Mukai, T., Nishida, A., and Kokubun, S.: Structure and dynamics of magnetic reconnection for substorm onsets with Geotail observations, J. Geophys. Res., 103, 4419-4440, 1998.

Nagai, T., Singer, H. J., Mukai, T., Yamamoto, T., and Kokubun, S.: Development of substorms in the near-Earth tail, Adv. Space Res., 25, 1651-1662, 2000.

Nagai, T., Shinohara, I., Fujimoto, M., Hoshino, M., Saito, Y., Machida, S., and Mukai, T.: Geotail observations of the Hall current system: Evidence of magnetic reconnection in the magnetotail, J. Geophys. Res., 106, 25 929-25 950, 2001.

Nagai, T., Shinohara, I., Fujimoto, M., Machida, S., Nakamura, R., Saito, Y., and Mukai, T.: Structure of the Hall current system in the vicinity of the magnetic reconnection site, J. Geophys. Res., 108, 1357, doi:10.1029/2003JA009900, 2003.

Nakagawa, T. and Nishida, A.: Southward magnetic field in the neutral sheet produced by wavy motions propagating in the dawndusk direction, Geophys. Res. Lett., 16, 1265-1268, 1989.

Nakamura, M. S., Matsumoto, H., and Fujimoto, M.: Interchange instability at the leading part of reconnection jets, Geophys. Res. Lett., 29, 1247, doi:10.1029/2001GL013780, 2002.

Nakamura, R., Baumjohann, W., Brittnacher, M., Sergeev, V. A., Kubyshkina, M., Mukai, T., and Liou, K.: Flow bursts and auroral activations: Onset timing and foot point location, J. Geophys. Res., 106, 10 777-10 789, 2001a.

Nakamura, R., Baumjohann, W., Schödel, R., Brittnacher, M., Sergeev, V. A., Kubyshkina, M., Mukai, T., and Liou, K.: Earthward flow bursts, auroral streamers, and small expansions, J. Geophys. Res., 106, 10 791-10 802, 2001b.

Nakamura, R., Baumjohann, W., Mouikis, C., Kistler, L. M., Runov, A., Volwerk, M., Asano, Y., Vörös, Z., Zhang, T. L., Klecker, B., Rème, H., and Balogh, A.: Spatial scale of high-speed flows in the plasma sheet observed by Cluster, Geophys. Res. Lett., 31, L09894, doi:10.1029/2004GL019558, 2004a.

Nakamura, R., Baumjohann, W., Nagai, T., Fujimoto, M., Mukai, T., Klecker, B., Treumann, R., Balogh, A., Rème, H., Sauvaud, J.-A., Kistler, L., Mouikis, C., Owen, C. J., Fazakerley, A. N., Dewhurst, J. P., and Bogdanova, Y.: Flow shear near the boundary of the plasma sheet observed by Cluster and Geotail, J. Geophys. Res, 109, A05204, doi:10.1029/2003JA010174, 2004b.

Nakamura, R., Amm, O., Laakso, H., Draper, N., Lester, M., Grocott, A., Klecker, B., McCrea, I. W., Balogh, A., Rème, H., and André, M.: Localized fast flow disturbance observed in the plasma sheet and in the ionosphere, Ann. Geophys., 23, 553566,2005 , http://www.ann-geophys.net/23/553/2005/.

Ohtani, S. I. and Raeder, J.: Tail current surge: New insights from a global MHD simulation and comparison with satel- 
lite observations, J. Geophys. Res., 109, A01207, doi:10.1029/ 2002JA009750, 2004.

Ohtani, S., Kokubun, S., Elphic, R. C., and Russell, C. T.: Fieldaligned current signatures in the near-tail region, 1, ISEE observations in the plasma sheet boundary layer, J. Geophys. Res., 93, 9709-9720, 1988.

Ohtani, S., Takahashi, K., Zanetti, I. J., Potemra, T. A., McEntire, R. W., and Iijima, T.: Tail current disruption in the geosynchronous region, in: Magnetospheric substorms, Geophys Monogr. Ser. Vol 64, edited by: Kan, J. R., Potemta, T. A., Kokubun, S., and Iijima, T., pp. 131-137, AGU, Washington, DC, 1991.

Ohtani, S., Kokubun, S., and Russell, C. T.: Radial expansion of the tail current disruption during substorms: A new approach to the substorm onset region, J. Geophys. Res., 97, 3129-3136, 1992.

Ohtani, S., Higuchi, T., Lui, A. T. Y., and Takahashi, K.: Magnetic fluctuations associated with tail current disruption: Fractal analysis, J. Geophys. Res., 100, 19 135-19 145, 1996.

Ohtani, S. I., Shay, M. A., and Mukai, T.: Temporal structure of the fast convective flow in the plasma sheet: Comparison between observations and two-fluid simulations, J. Geophys. Res., 109, A03210, doi:10.1029/2003JA010002, 2004.

Øieroset, M., Phan, T. D., Fujimoto, M., Lin, R. P., and Lepping, R. P.: In situ detection of collisionless reconnection in the Earth's magnetotail, Nature, 412, 414-417, 2001.

Onsager, T. G., Thomsen, M. F., Gosling, J. T., and Bame, S. J.: Electron distributions in the plasma sheet boundary layer: Timeof-flight effects, Geophys. Res. Lett., 17, 1837-1840, 1990.

Owen, C. J. and Slavin, J. A.: Energetic ion events associated with traveling compression regions, in: Proceedings of International Conference on Substorms, pp. 365-370, ESA SP-335, 1992.

Owen, C. J., Slavin, J. A., Fazakerley, A. N., Danlop, M. W., and Balogh, A.: Cluster electron observations of the separatrix layer during traveling compression regions, Geophys. Res. Lett, 32, L03104, doi:10.1029/2004GL021767, 2005.

Parker, E.: Sweet's mechanism for merging magnetic field in conducting fluids, J. Geophys.Res., 62, 509-520, 1957.

Paschmann, G., Schwartz, S. J., Escoubet, C. P., and S. Haaland (Eds.): Outer Magnetospheric Boundaries: Cluster Results Series, Space Sciences Series of ISSI, vol. 20, ISSI, Bern, Switzerland, 2005.

Pellat, R., Coroniti, F. V., and Pritchett, P. L.: Does ion tearing exist?, Geophys. Res. Lett, 18, 143-146, 1991.

Petrukovich, A. A., Baumjohann, W., Nakamura, R., Schödel, R., and Mukai, T.: Are earthward bursty bulk flows convective or field-aligned?, J. Geophys. Res., 106, 21 211-21 215, 2001.

Petrukovich, A. A., Baumjohann, W., Nakamura, R., Balogh, A., Mukai, T., Glassmeier, K.-H., Rème, H., and Klecker, B.: Plasma sheet structure during strongly northward IMF, J. Geophys. Res., 108, 1258, doi:10.1029/2002JA009738, 2003.

Petrukovich, A. A., Zhang, T. L., Baumjohann, W., Nakamura, R., Runov, A., Balogh, A., and Carr, C.: Oscillatory magnetic flux tube slippage in the plasma sheet, Ann. Geophys., 24, 16951704, 2006,

http://www.ann-geophys.net/24/1695/2006/.

Petschek, H. E.: Magnetic field annihilation, in: AAS/NASA Symposium on the Physics of Solar Flares SP-50, W. N. Ness (Editor) , pp. 425-439, NASA, Washington, D.C., 1964.

Pritchett, P. L.: Collisionless magnetic reconnection in a three- dimensional open system, J. Geophys. Res., 106, 25 961-25 978, 2001.

Raeder, J.: Global Magnetohydrodynamics - A Tutorial, in: Space Plasma Simulations, J. Buechner, C. Dum and M. Scholer (Editors), p. 212-246, Springer, Berlin, 2003.

Raj, A., Phan, R., Lin, R. P., and Angelopoulos, V.: Wind survey of high-speed bulk flows and field-aligned beams in the nearEarth plasma sheet, J. Geophys. Res., 107, 1419, doi:10.1029/ 2001JA007547, 2002.

Richardson, I. G., Owen, C. J., and Slavin, J. A.: Energetic ( $>0.2$ $\mathrm{MeV}$ ) electron bursts in the deep geomagnetic tail observed by the Goddard Space Flight Center experiment on ISEE 3: Association with geomagnetic substorms, J. Geophys. Res., 101, 2723-2740, 1996.

Roux, A., Perraut, S., Robert, P., Morane, A., Pedersen, A., Korth A., Kremser, G., Aparicio, B., Rodgers, D., and Pellinen, R.: Plasma sheet instability related to the westward traveling surge, J. Geophys. Res., 96, 17 697-17 714, 1991.

Runov, A., Nakamura, R., Baumjohann, W., Treumann, R. A., Zhang, T. L., Volwerk, M., Vörös, Z., Balogh, A., Glassmeier, K.-H., Klecker, B., Rème, H., and Kistler, L.: Current sheet structure near magnetic X-line observed by Cluster, Geophys. Res. Lett., 30, 1579, doi:10.1029/2002GL016730, 2003a.

Runov, A., Nakamura, R., Baumjohann, W., Zhang, T. L., Volwerk, M., Eichelberger, H.-U., and Balogh, A.: Cluster observation of a bifurcated current sheet, Geophys. Res. Lett., 30, 1036, doi: 10.1029/2002GL016136, 2003b.

Runov, A., Sergeev, V. A., Baumjohann, W., Nakamura, R., Apatenkov, S., Asano, Y., Volwerk, M., Vörös, Z., Zhang, T. L., Petrukovich, A., Balogh, A., Sauvaud, J.-A., Klecker, B., and Rème, H.: Electric current and magnetic field geometry in flapping magnetotail current sheets, Ann. Geophys., 23, 1391-1403, 2005 , http://www.ann-geophys.net/23/1391/2005/.

Runov, A., Sergeev, V. A., Nakamura, R., Baumjohann, W., Apatenkov, S., Asano, Y., Takada, T., Volwerk, M., Vörös, Z., Zhang, T. L., Sauvaud, J.-A., Rème, H., and Balogh, A.: Local structure of the magnetotail current sheet: 2001 Cluster observations, Ann. Geophys., 24, 247-262, 2006, http://www.ann-geophys.net/24/247/2006/.

Sagdeev, R. Z. and Shapiro, V. D.: Influence of Transverse Magnetic Field on Landau Damping, JETP Lett., 17, 279-282, 1973.

Sarris, E. T., Krimigis, S. M., and Armstrong, T. P.: Observations of magnetospheric bursts of high-energy protons and electrons at approximately 35 Earth radii with Imp 7, J. Geophys. Res., 81, 2341-2355, 1976

Sauvaud, J.-A.: A multisatellite study of the electromagnetic field changes associated with substorms in the tail lobe, Ann. Geophys., submitted, 2008.

Sauvaud, J.-A. and Kovrazhkin, R. A.: Two types of energydispersed ion structures at the plasma sheet boundary, J. Geophys. Res., 109, A12213, doi:10.1029/2003JA010333, 2004.

Sauvaud, J.-A. and Winckler, J. R.: Dynamics of plasma, energetic particles, and fields near synchronous orbit in the nighttime sector during magnetospheric substorms, J. Geophys. Res., 85, 2043-2056, 1980

Sauvaud, J.-A., Jacquey, C., Beutier, T., Owen, C., Lepping, R. P., Russell, C. T., and Belian, R. J.: Large scale dynamics of the magnetospheric tail induced by substorms: A multisatellite 
study, J. Geom. Geoelec., 48, 675-686, 1996.

Sauvaud, J.-A., Popescu, D., Delcourt, D. C., Parks, G. K., Brittnacher, M., Sergeev, V. A., Kovrazhkin, R. A., Mukai, T., and Kokubun, S.: Sporadic plasma sheet ion injections into the high altitude auroral bulge - satellite observations, J. Geophys. Res., 104, 28 565-28 586, 1999.

Schindler, K.: A theory of the substorm mechanism, J. Geophys. Res., 79, 2803-2810, 1974.

Schindler, K., Hesse, M., and Birn, J.: General magnetic reconnection, parallel electric fields, and helicity, J. Geophys. Res., 93, 5547-5557, 1988.

Schödel, R., Baumjohann, W., Nakamura, R., Sergeev, V. A., and Mukai, T.: Rapid flux transport in the central plasma sheet, J. Geophys. Res., 106, 301-313, 2001.

Seboldt, W.: Nonlocal analysis of low-frequency waves in the plasma sheet, J. Geophys. Res., 95, 10 471-10 479, 1990.

Semenov, V. S., Heyn, M. F., and Kubyshkin, I. V.: Time-Dependet Reconnection of the Magnetic Field Lines, Sov. Astron., 60, 1138-1147, 1983.

Semenov, V. S., Farrugia, C. J., Biernat, H. K., et al.: Reconnection associated surface waves at the magnetopause, Geophys. Res. Lett., 21, 2437-2440, 1994.

Semenov, V. S., Heyn, M. F., and Ivanov, I. B.: Magnetic reconnection with space and time varying reconnection rates in a compressible plasmas, Phys. Plasmas, 11, 62-70, 2004a.

Semenov, V. S., Kubyshkin, I. V., Rijnbeek, R. P., and Biernat, H. K.: Analytical theory of unsteady Petschek-type reconnection, in: Physics of Magnetic Reconnection in High-Temerature Plasmas, edited by: Ugai, M., pp. 35-68, Research Signpost, Trivandrum, India, 2004b.

Semenov, V. S., Penz, T., Heyn, M. F., Ivanov, I. B., Kubyshkin, I. V., Biernat, H. K., Ivanova, V. V., and Rijnbeek, R. P.: Reconstruction of the reconnection rate from magnetic field disturbances in an incompressible plasma, in: Solar-planetary relations, edited by: Biernat, H. K., Lammer, H., Vogl, D. F., and Mühlbachler, S., pp. 261-302, Research Signpost, Trivandrum, India, 2005.

Sergeev, V. A.: Bursty Bulk Flows and their ionospheric footprints, in: Multiscale Processes in the Earth's Magnetosphere: From Interball to Cluster, edited by: Sauvaud, J.-A. and Nemecek, Z., pp. 289-306, Kluver Academic Publisher, Netherlands, 2004.

Sergeev, V. A.: Bursty Bulk Flows and their ionospheric footprints, in: Multiscale Processes in the Earth's Magnetosphere: From Interball to Cluster, J.-A. Sauvaud, Z. Nemecek (Editors), pp. 289-306, Kluver Academic Publisher, Netherlands, 2004.

Sergeev, V. A., Semenov, V. S., and Sidneva, M. V.: Impulsive reconnection in the magnetotail during substorm expansion, Planet. Space Sci., 35, 1199-1212, 1987.

Sergeev, V., Elphic, R. C., Mozer, F. S., Saint-Marc, A., and Sauvaud, J.-A.: A two-satellite study of nightside flux transfer events in the plasma sheet, Planet. Space Sci., 40, 1551-1572, 1992.

Sergeev, V. A., Angelopulous, V., Gosling, J. T., Cattell, C. A., and Russell, C. T.: Detection of localized,plasma-depleted flux tubes or bubbles in the midtail plasma sheet, J. Geophys. Res., 101, 10 817-10 826, 1996.

Sergeev, V. A., Sauvaud, J.-A., Popescu, D., Kovrazhkin, R. A., Liou, K., Newell, P., Brittnacher, M., G. Parks, R. N., Mukai, T., and Reeves, G. D.: Multiple-spacecraft observation of a narrow transient plasma jet in the Earth's plasma sheet, Geophys. Res. Lett., 27, 851-855, 2000a.

Sergeev, V. A., Sauvaud, J.-A., Popescu, D., Kovrazhkin, R. A., Lutsenko, V. N., Zelenyi, L. M., Syrjasuo, M., Viljanen, A., Pulkkinen, T. I., Kudela, K., Kokubun, S., and Mukai, T.: Plasma sheet ion injections into the auroral bulge: Correlative study of spacecraft and ground observations, J. Geophys. Res., 105, 18 465-18 482, 2000b.

Sergeev, V. A., Baumjohann, W., and Shiokawa, K.: Bi-directional electron distributions associated with near-tail flux transport, Geophys. Res. Lett., 28, 3813-3816, 2001.

Sergeev, V., Liou, K., Newell, P. T., Ohtani, S.-I., Hairston, M. R., and Rich, F.: Auroral streamers: Characteristics of associated precipitation, convection and field-aligned currents, Ann. Geophys., 22, 537-548, 2004a, http://www.ann-geophys.net/22/537/2004/.

Sergeev, V., Runov, A., Baumjohann, W., Nakamura, R., Zhang, T. L., Balogh, A., Louarn, P., Sauvaud, J.-A., and Rème, H.: Orientation and propagation of current sheet oscillations, Geophys. Res. Lett., 31, L05807, doi:10.1029/2003GL019346, 2004 b.

Sergeev, V. A., Kubyshkina, M. V., Baumjohann, W., Nakamura, R., Amm, O., Pulkkinen, T., Angelopoulos, V., Mende, S. B., Klecker, B., Nagai, T., Sauvaud, J.-A., Slavin, J. A., and Thomsen, M. F.: Transition from substorm growth to substorm expansion phase as observed with a radial configuration of ISTP and Cluster spacecraft, Ann. Geophys., 23, 2183-2198, 2005, http://www.ann-geophys.net/23/2183/2005/.

Sergeev, V. A., Sormakov, D. A., Apatenkov, S. V., Baumjohann, W., Nakamura, R., Runov, A., Mukai, T., and Nagai, T.: Survey of large-amplitude flapping motions in the midtail current sheet, Ann. Geophys., 24, 2015-2024, 2006, http://www.ann-geophys.net/24/2015/2006/.

Sergeev, V., Semenov, V., Kubyshkina, M., Ivanova, V., Baumjohann, W., Nakamura, R., Penz, T., Runov, A., Zhang, T., Glassmeier, K., Angelopoulos, V., Frey, H., Sauvaud, J., Daly, P., Cao, J., Singer, H., and Lucek, E.: Observation of repeated intense near-Earth reconnection on closed field lines with Cluster, Double Star and other spacecraft, Geophys. Res. Lett., 34, L0213, doi:10.1029/2006GL028452, 2007.

Shao, X., Sharma, A. S., Chen, J.: Bursty Bulk Flows (BBF) related features during magnetospheric substorm: An integrated view from global MHD simulations, AGu Fall Meeting, Abs. SMSM51B-1406, 2006.

Sharma, A. S., Baker, D. N., and Borovsky, J. E.: Nonequilibrium phenomena in the magnetosphere: Phase transition, selforganized criticality and turbulence, in: Nonequilibrium Phenomena in Plasmas, edited by: Sharma, A. S. and Kaw, P. K., pp. 3-22, Springer, 2005a.

Sharma, A. S., Ukhorskiy, A. Y., and Sitnov, M. I.: Global and multiscale phenomena of the magnetosphere, in: Nonequilibrium Phenomena in Plasams, edited by: Sharma, A. S. and Kaw, P. K., pp. 117-144, Springer, 2005b.

Sharma, A. S., Zelenyi, L. M., Malova, H. V., Popov, V. Y., and Delcourt, D. C.: Multiscale structure of thin current sheets: Multiscale "Matreshka" model, in: Proc. 8 th Int. Conf. Substorms, M. Syrjasuo and E. Donovan (Editors), pp. 117-144, University of Calgary, Calgary, Canada, 2006.

Shay, M. A., Drake, J. F., Rogers, B. N., and Denton, R. E.: The scaling of collisionless, magnetic reconnection for large systems, 
Geophys. Res. Lett, 26, 2163-2166, 1999.

Shay, M. A., Drake, J. F., Swisdak, M., Dorland, W., and Rogers, B. N.: Inherently three dimensional magnetic reconnection: A mechanism for bursty bulk flows?, Geophys. Res. Lett., 30, 1345, doi:10.1029/2002GL016267, 2003.

Shirai, H., Takada, T. K., Kamide, Y., and Mukai, T.: Enhancements of lobe ion density and velocity associated with plasmoids, J. Geophys. Res., 106, 29 935-29942, 2001.

Shirataka, N., Fujimoto, M., Hasegawa, H., and TanDokoro, R.: Reproducing the bi-polar magnetic signature at the jet leading edge by 3-D reconnection with non-zero guide field, J. Geophys. Res., 111, A07201, doi:10.1029/2005JA011521, 2006.

Sibeck, D. G., Siscoe, G. L., Slavin, J. A., Smith, E. J., Bame, S. J., and Scarf, F. L.: Magnetotail flux ropes, Geophys. Res. Lett., 11, 1090-1093, 1984.

Siscoe, G.: The magnetosphere: A union of interdependent parts, EOS Trans. AGU, 72, 494-495, 1991.

Siscoe, G., Erickson, G. M., Sonnerup, B. U. O., Maynard, N. C., Siebert, K. D., Weimer, D. R., and White, W. W.: Global role of $E_{\|}$in magnetopause reconnection: An explicit demonstration, J. Geophys. Res., 106, 13 015-13 022, 2001.

Sitnov, M. I., Malova, H. V., and Sharma, A. S.: Role of the temperature ratio in the linear stability of the quasi-neutral sheet tearing mode, Geophys. Res. Lett., 25, 269-272, 1998.

Sitnov, M. I., Zelenyi, L. M., Malova, H. V., and Sharma, A. S.: Thin current sheet embedded within a thicker plasma sheet: Selfconsistent kinetic theory, J. Geophys. Res., 105, 13 029-13 044, 2000

Sitnov, M. I., Swisdak, M., Drake, J. F., Guzdar, P. N., and Rogers, B. N.: A model of the bifurcated current sheet: 2 . Flapping motion, Geophys. Res. Lett., 31, L09805, doi:10.1029/ 2004GL019473, 2004.

Sitnov, M. I., Swisdak, M., Guzdar, P. N., and Runov, A.: Structure and dynamics of a new class of thin current sheets, J. Geophys. Res., 111, A08204, doi:10.1029/2005JA011517, 2005.

Slavin, J. A., Smith, E. J., Tsurutani, B. T., Sibeck, D. G., Singer, H. J., Baker, D. N., Gosling, J. T., Hones, E. W., and Scarf, F. L.: Substorm associated traveling compression regions in the distant tail - ISEE-3 geotail observations, Geophys. Res. Lett., 11, 657660, 1984.

Slavin, J. A., Baker, D. N., Fairfield, D. H., Craven, J. D., Frank, L. A., Elphic, R. C., Galvin, A. B., Hughes, W. J., Manka, R. H., and Smith, E. J.: CDAW-8 observations of plasmoid signatures in the geomagnetic tail: An assessment, J. Geophys. Res., 94, 15 153-15 175, 1989.

Slavin, J. A., Smith, M. F., Mazur, E. L., Baker, D. N., Iyemori, T., Singer, H. J., and Greenstadt, E. W.: ISEE 3 plasmoid and TCR observations during an extended interval of substorm activity, Geophys. Res. Lett., 19, 825-828, 1992.

Slavin, J. A., Smith, M. F., Mazur, E. L., Baker, D. N., Hones Jr., E. W., Iyemori, T., and Greenstadt, E. W.: ISEE 3 observations of traveling compression regions in the Earth's magnetotail, J. Geophys. Res., 98, 15 425-15 446, 1993.

Slavin, J. A., Owen, C. J., Kuznetsova, M. M., and Hesse, M.: ISEE 3 observations of plasmoids with flux rope magnetic topologies, Geophys. Res. Lett., 22, 2061-2064, 1995.

Slavin, J. A., Fairfield, D. H., Kuznetsova, M. M., Owen, C. J., Lepping, R. P., Taguchi, S., Mukai, T., Saito, Y., Yamamoto, T., Kokubun, S., Lui, A. T. Y., and Reeves, G. D.: ISTP observations of plasmoid ejection: IMP 8 and Geotail, J. Geophys. Res., 103, 119-134, 1998.

Slavin, J. A., Hesse, M., Owen, C. J., Taguchi, S., Fairfield, D. H., Lepping, R. P., Kokubun, S., Mukai, T., Lui, A. T. Y., Anderson, R. R., Matsumoto, H., and Sutcliffe, P. R.: Dual spacecraft observations of lobe magnetic field perturbations before, during and after plasmoid release, Geophys. Res. Lett., 26, 2897-2900, 1999.

Slavin, J. A., Fairfield, D. H., Lepping, R. P., Hesse, M., Ieda, A., Tanskanen, E., Østgaard, N., Mukai, T., Nagai, T., Singer, H. J., and Sutcliffe, P. R.: Simultaneous observations of earthward flow bursts and plasmoid ejection during magnetospheric substorms, J. Geophys. Res., 107, 1106, doi:10.1029/2000JA003501, 2002.

Slavin, J. A., Lepping, R. P., Gjerloev, J., Fairfield, D. H., Acuna, M. H., Goldstein, M. L., Balogh, A., Dunlop, M., Kivelson, M. G., Khurana, K., Fazakerley, A., Owen, C. J., Rème, H., and Bosqued, J. M.: Cluster measurements of electric current density within a flux rope in the plasma sheet, Geophys. Res. Lett., 30, 1362, doi:10.1029/2003GL016411, 2003a.

Slavin, J. A., Lepping, R. P., Gjerloev, J., Fairfield, D. H., Hesse, M., Owen, C. J., Moldwin, M. B., Nagai, T., Ieda, A., and Mukai, T.: Geotail observations of magnetic flux ropes in the plasma sheet, J. Geophys. Res., 108, 1015, doi:10.1029/2002JA009557, $2003 b$.

Slavin, J. A., Owen, C. J., Dunlop, M. W., Borälv, E., Moldwin, M. B., Sibeck, D. G., Tanskanen, E., Goldstein, M. L., Fazakerley, A., Balogh, A., Lucek, E., Richter, I., Rème, H., and Bosqued, J. M.: Cluster four spacecraft measurements of small traveling compression regions in the near-tail, Geophys. Res. Lett., 30, 2208, doi:10.1029/2003GL018438, 2003c.

Slavin, J. A., Tanskanen, E. I., Hesse, M., Owen, C. J., Dunlop, M. W., Imber, S., Lusek, E. A., Balogh, A., and Glassmeier, K.-H.: Cluster observations of travelling compression regions in the near-tail, J. Geophys. Res, 30, A06207, doi: 10.1029/2004JA010878, 2005.

Smith, J. M., Roberts, B., and Oliver, R.: Magnetoacoustic wave propagation in current sheets, Astron. Astrophys., 327, 377-387, 1997.

Snekvik, K., Haaland, S., Østgaard, N., Hasegawa, H., Nakamura, R., Takada, T., Pitout, F., Rème, H., Klecker, B., and Lucek, E.: Cluster observation of a field-aligned current at the dawn flank of a bursty bulk flow, Ann. Geophys., 25, 1405-1415, 2007, http://www.ann-geophys.net/25/1405/2007/.

Sonnerup, B.: Solar System Plasma Physics, vol. III, NorthHolland, New York, 1979.

Sonnerup, B. U. Ö.: On the theory of steady state reconnection, Comput. Phys. Comm., 49, 143-159, 1988.

Sonnerup, B. U. Ö., Hasegawa, H., Teh, W.-L., and Hau, L.-N.: Grad-Shafranov reconstruction: An overview, J. Geophys. Res. 111, A09204, doi:10.1029/2006JA011717, 2006.

Speiser, T. W.: Particle Trajectories in Model Current Sheets, 1, Analytical Solutions, J. Geophys. Res., 70, 4219-4226, 1965.

Speiser, T. W. and Ness, N. F.: The neutral sheet in the geomagnetic tail: Its motion, equivalent currents, and field line reconnection through it, J. Geophys. Res., 72, 131-141, 1967.

Taguchi, S., Slavin, J. A., Kiyohara, M., Nose, M., Reeves, G. D., and Lepping, R. P.: Temporal relationship between midtail traveling compression regions and substorm onset: Evidence for near-Earth neutral line formation in the late growth phase, J. Geo- 
phys. Res., 103, 26 607-26612, 1998a.

Taguchi, S., Slavin, J. A., and Lepping, R. P.: Traveling compression regions in the midtail: Fifteen years of IMP 8 observations, J. Geophys. Res., 103, 17 641-17 650, 1998b.

TanDokoro, R. and Fujimoto, M.: Three-dimensional MHD study of the structure at the leading part of a reconnection jet, Geophys. Res. Lett, 110, L23102, doi:10.1029/2005GL024467, 2005.

Taylor, J. B.: Relaxation and magnetic reconnection in plasmas, Rev. Mod. Phys., 58, 741-763, 1986.

Terasawa, T.: Hall current effect on tearing mode instability, Geophys. Res. Lett, 10, 475-478, 1983.

Terasawa, T. and Nishida, A.: Simultaneous observations of relativistic electron bursts and neutral-line signatures in the magnetotail, Planet. Space. Sci, 24, 855-866, 1976.

Treumann, R. A., Jaroschek, C. H., Nakamura, R., Runov, A., and Scholer, M.: The role of the Hall effect in collisionless magnetic reconnection, Adv. Space. Res., 38, 101-111, 2006.

Ueno, G., Ohtani, S.-I., Mukai, T., Saito, Y., and Hayakawa, H.: Hall current system around the magnetic neutral line in the magnetotail: Statistical study, J. Geophys. Res., 108, 1347, doi: 10.1029/2002JA009733, 2003.

Ugai, M. and Zheng, L.: Modeling of traveling compression regions in the Earth's magnetotail by the spontaneous fast reconnection model, Phys. Plasmas, 13, 032 901-032 901-7, 2006a.

Ugai, M. and Zheng, L.: Parametric studies on traveling compression regions observed in the Earth's magnetotail, Phys. Plasmas, 13, 062 906-062 906-7, 2006b.

Ukhorskiy, A. Y., Sitnov, M. I., Sharma, A. S., and Papadopoulos, K.: Global and multiscale dynamics of the magnetosphere: From modeling to forecasting, Geophys. Res. Lett., L08802, doi:10. 1029/2003GL018932, 2004.

Uritsky, V. M., Klimas, A. J., Vassiliadis, D., Chua, D., and Parks, G. D.: Scale-free statistics of spatiotemporal auroral emissions as depicted by POLAR UVI images: The dynamic magnetosphere is an avalanching system, J. Geophys. Res., 107, 1426, doi:10.1029/2001JA000281, 2002.

Vasyliunas, V. M.: Theoretical models of magnetic field line merging. I., Rev. Geophys. Space Phys., 13, 303-336, 1975.

Volwerk, M., Glassmeier, K.-H., Runov, A., Nakamura, R., Baumjohann, W., Klecker, B., Richter, I., Balogh, A., Rème, H., and Yumoto, K.: Flow burst induced large scale plasma sheet oscillation, J. Geophys. Res., 109, A11208, doi:10.1029/ 2004JA010533, 2004.

Vörös, Z., Baumjohann, W., Nakamura, R., Runov, A., Zhang, T. L., Volwerk, M., Eichelberger, H.-U., Balogh, A., Horbury, T. S., Glassmeier, K.-H., Klecker, B., and Rème, H.: Multi-scale magnetic field intermittence in the plasma sheet, Ann. Geophys., 21, 1955-1964, 2003,

http://www.ann-geophys.net/21/1955/2003/.

Walthour, D. W., Gosling, J. T., Sonnerup, B. U. O., and Russell, C. T.: Observation of anomalous slow-mode shock and reconnection layer in the dayside magnetospause, J. Geophys. Res., 99, $23705-23722,1994$.

Wilken, B., Zong, Q. G., Daglis, I. A., Doke, T., Livi, S., Maezawa, K., Pu, Z. Y., Ullaland, S., and Yamamoto, T.: Tailward flowing energetic oxygen-ion bursts associated with multiple flux ropes in the distant magnetotail: Geotail observations, Geophys. Res. Lett., 22, 3267-3270, 1995.

Wiltberger, M., Pulkkinen, T. I., Lyon, J. G., and Goodrich, C. C.:
MHD Simulation of the Magnetotail During the Dec. 10, 1997 Substorm, J. Geophys. Res., 105, 27 649-27 663, 2000.

Wolf, R. A., Kumar, V., Toffoletto, F. R., Erickson, G. M., Savoie, A. M., Chen, C. X., and Lemon, C. L.: Estimating local plasma sheet PV5/3 from single spacecraft observations, J. Geophys. Res., 111, A12218, doi:10.1029/2006JA012010, 2006.

Wygant, J. R., Keiling, A., Cattell, C. A., Johnson, M., Lysak, R. L., Temerin, M., Mozer, F. S., Kletzing, C. A., Scudder, J. D., Peterson, W., Russell, C. T., Parks, G., Brittnacher, M., Germany, G., and Spann, J.: Polar spacecraft based comparison of intense electric fields and Poynting flux near and witin the plasma sheet-tail lobe boundary to UVI images: An energy source for the aurora, J. Geophys. Res., 105, 18 675-18 692, 2000.

Wygant, J. R., Keiling, A., Cattell, C. A., Lysak, R. L., Temerin, M., Mozer, F. S., Kletzing, C. A., Scudder, J. D., Streltsov, V., Lotko, W., and Russell, C. T.: Evidence for kinetic Alfvén waves and parallel electron energization at 4-6 $R_{E}$ altitudes in the plasma sheet boundary layer, J. Geophys. Res., 107, 1201, doi:10.1029/ 2001JA900113, 2002.

Wygant, J. R., Cattell, C. A., Lysak, R., Song, Y., Dombeck, J., McFadden, J., Mozer, F. S., Carlson, C. W., Parks, G., Lucek, E. A., Balogh, A., Andre, M., Rème, H., Hesse, M., and Mouikis, C.: Cluster observations of an intense normal component of the electric field at a thin reconnecting current sheet in the tail and its role in the shock-like acceleration of the ion fluid into the separatrix region, J. Geophys. Res., 110, A09206, doi:10.1029/ 2004JA010708, 2005.

Xiao, C. J., Pu, Z. Y., Ma, Z. W., Fu, S. Y., Huang, Z. Y., and Zong, Q. G.: Inferring of flux rope orientation with the minimum variance analysis technique, J. Geophys. Res., 109, A11218, doi: 10.1029/2004JA010594, 2004.

Yang, C. K. and Sonnerup, B. U. Ö.: Compressible magnetic field reconnection: A slow wave model, Astrophys. J., 206, 570-582, 1976.

Zaslavsky, G. M., Edelman, M., Guzdar, P. N., Sitnov, M. I., and Sharma, A. S.: Self-similarity and fractional kinetics of solar wind and magnetosphere coupling, Physica A, 373, 11-20, 2007.

Zelenyi, L. M. and Milovanov, A. V.: Fractal topology and strange kinetics: from percolation theory to problems in cosmic electrodynamics, Physics - Uspekhi, 47, 749-788, 2004.

Zelenyi, L. M., Kovrazhkin, R. A., and Bosqued, J. M.: Velocitydispersed ion beams in the nightside auroral zone: AUREOL-3 observations, J. Geophys. Res., 95, 12 119-12 139, 1990.

Zelenyi, L. M., Malova, H. V., Popov, V. Y., Delcourt, D., and Sharma, A. S.: Nonlinear equilibrium structure of thin current sheets: Influence of electron pressure anisotropy, Nonlin. Processes Geophys, 11, 579-587, 2004.

Zelenyi, L. M., Grigorenko, E. E., Sauvaud, J.-A., and Maggiolo, R.: Multiplet structure of acceleration processes in the distant magnetotail, Geophys. Res. Lett., 33, L06105, doi:10.1029/ 2005GL024901, 2006.

Zelenyi, L. M., Dolgonosov, M. S., Grigorenko, E. E., and Sauvaud, J.-A.: Universal scaling of non-adiabatic acceleration in magnetotail, JETP Lett., JETP Letters, 85, 187-193, 2007.

Zesta, E., Lyons, L. R., and Donovan, E.: The auroral signature of Earthward flow bursts observed in the magnetotail, Geophys. Res. Lett., 27, 3241-3244, 2000.

Zhang, T. L., Baumjohann, W., Nakamura, R., Balogh, A., and Glassmeier, K.-H.: A wavy twisted neutral sheet ob- 
served by Cluster, Geophys. Res. Lett., 29, 1899, doi:10.1029/ 2002GL015544, 2002.

Zhang, T. L., Nakamura, R., Volwerk, M., Runov, A., Baumjohann, W., Eichelberger, H. U., Carr, C., Balogh, A., Sergeev, V., Shi, J. K., and Fornacon, K.-H.: Double Star/Cluster observations of neutral sheet oscillations on 5 August 2004, Ann. Geophys., 23, 2909-2914, 2005,

http://www.ann-geophys.net/23/2909/2005/.

Zong, Q.-G., Wilken, B., Reeves, G. D., Daglis, I. A., Doke, T., Iyemori, T., Livi, S., Maezawa, K., T., T. M., Kokubun, S., Pu, Z.-Y., Ullaland, S., Woch, J., Lepping, R., and Yamamoto, T.: Geotail observations of energetic ion species and magnetic field in plasmoid-like structures in the course of an isolated substorm event, J. Geophys. Res., 102, 11 409-11 428, 1997.
Zong, Q.-G., Fritz, T. A., Pu, Z. Y., Fu, S. Y., Baker, D. N., Zhang, H., Lui, A. T., Vogiatzis, I., Glassmeier, K.-H., Korth, A., Daly, P. W., Balogh, A., and Reme, H.: Cluster observations of earthward flowing plasmoid in the tail, Geophys. Res. Lett., 31, L18803, doi:10.1029/2004GL020692, 2004.

Zwingmann, W.: Self-consistent magnetotail theory: Equilibrium structures including arbitrary variation along the tail axis, J. Geophys. Res., 88, 9101-9111, 1983. 